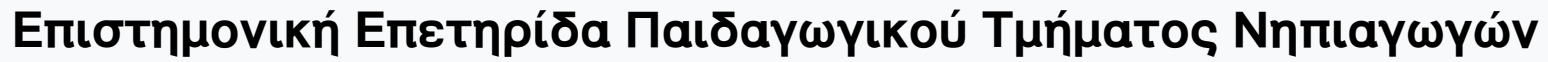 חaverıotnuíou Iwavvívwv
}

Tóp. 7 (2014)

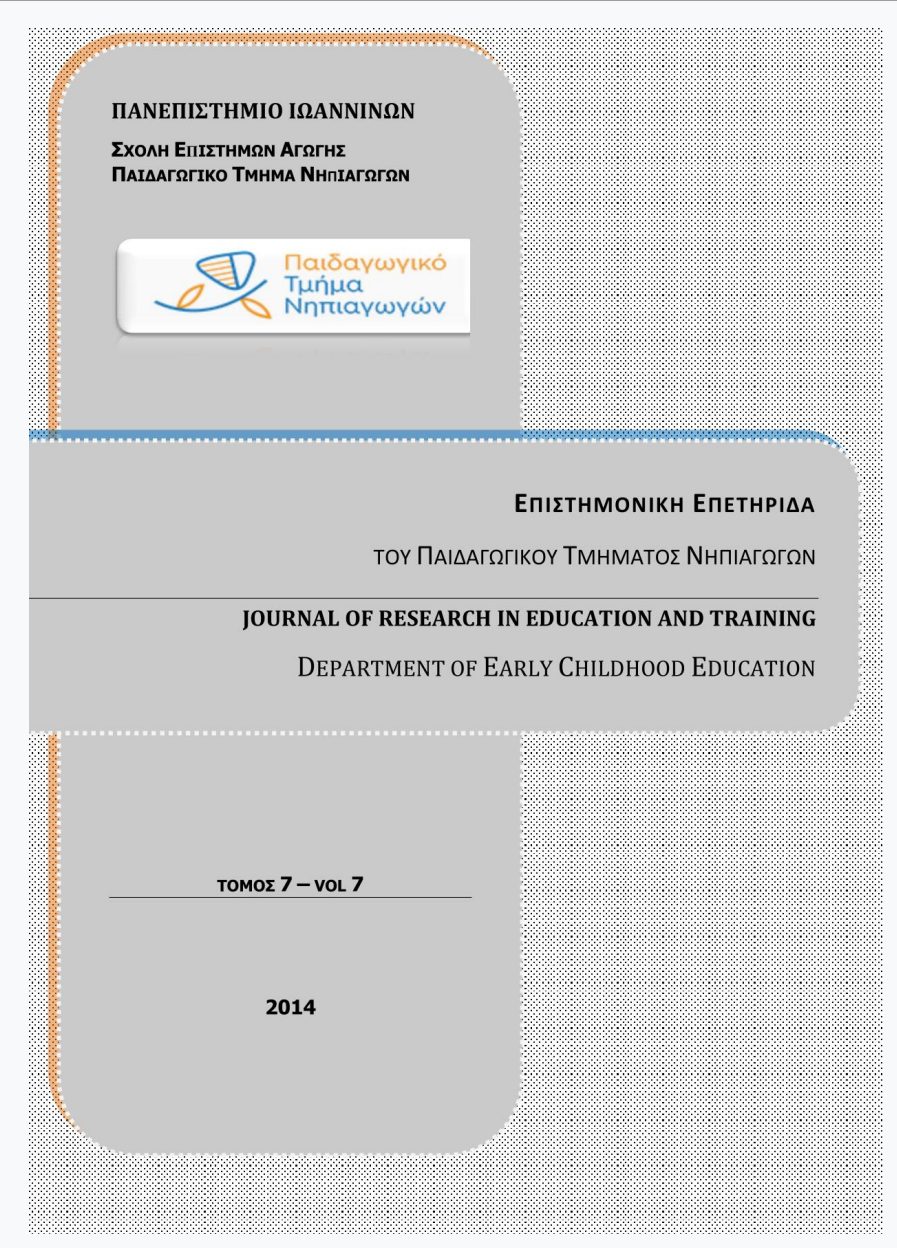

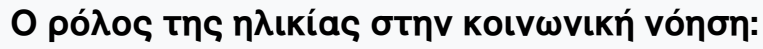

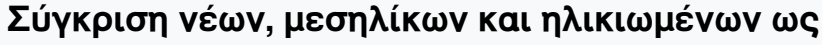

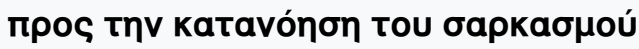

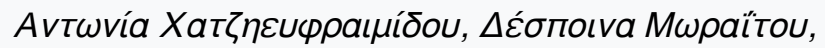

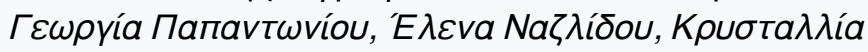
Пávtoıou

doi: $10.12681 /$ jret.854

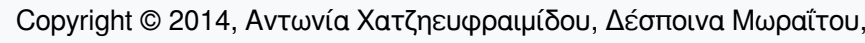

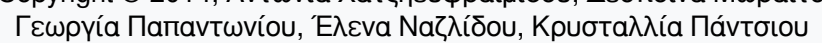

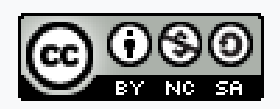

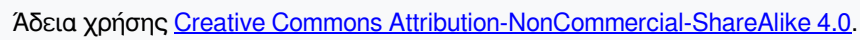

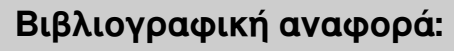

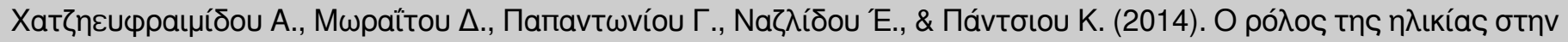

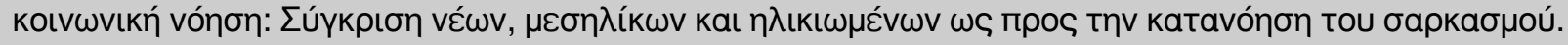

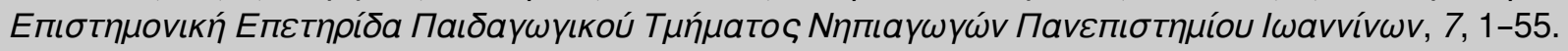
https://doi.org/10.12681/jret.854 


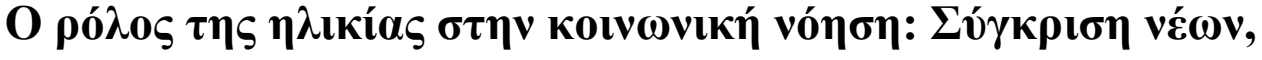

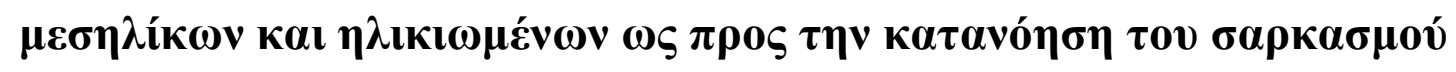

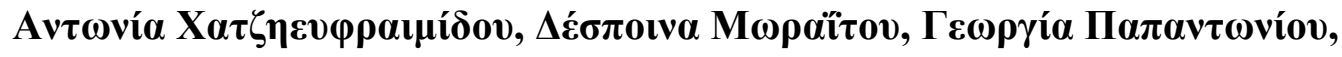

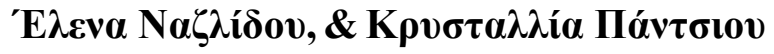

\section{ПЕРІАНЧН}

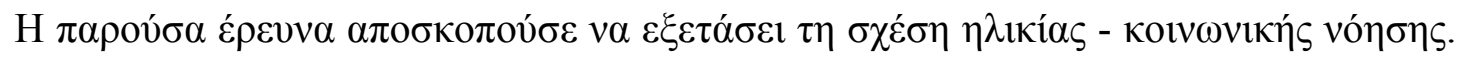

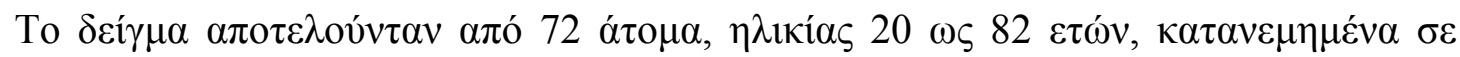

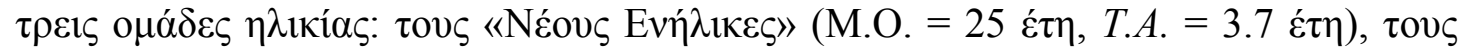

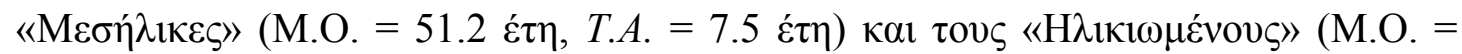

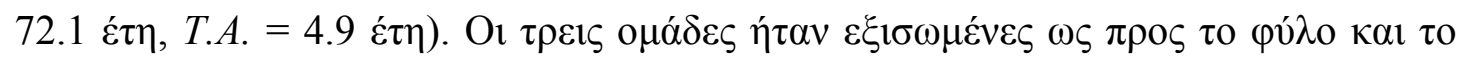

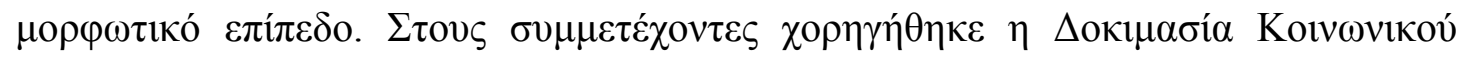
$\Sigma v \mu \pi \varepsilon \rho \alpha \sigma \mu o v ́(\mathrm{~A} \pi \lambda \mathrm{o} \varsigma)[\Delta \mathrm{K} \Sigma(\mathrm{A})$. Social Inference (Minimal) - SI(M): Part 2 of the

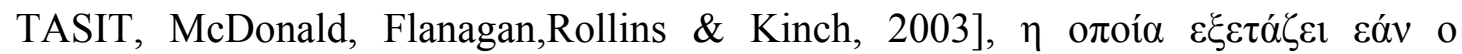

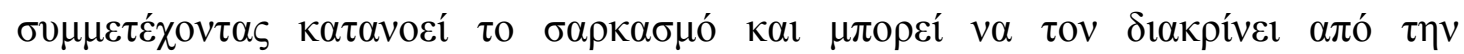

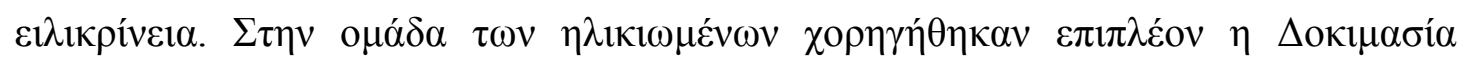

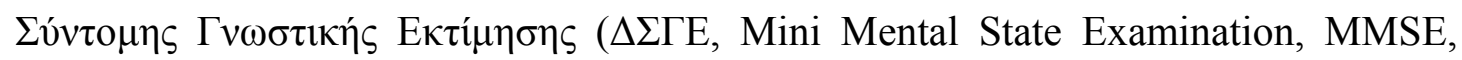

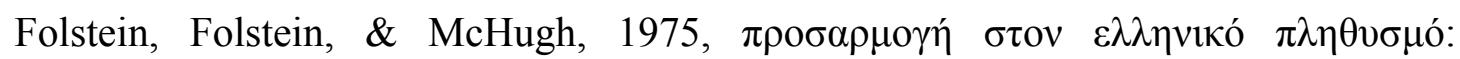

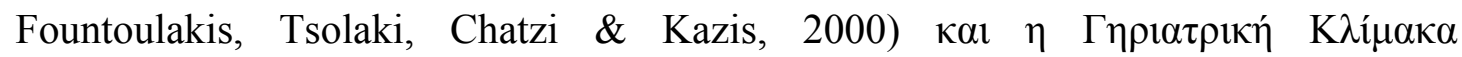

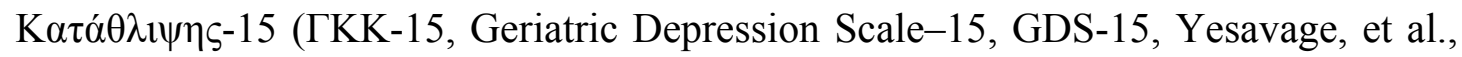

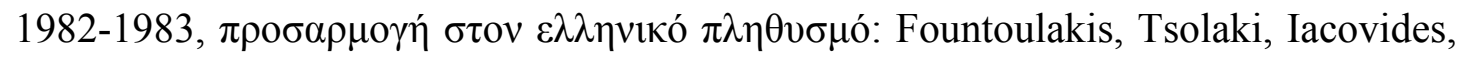

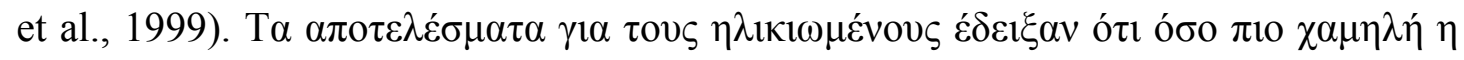

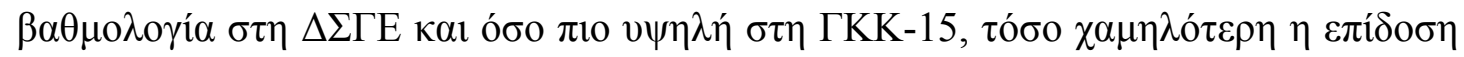

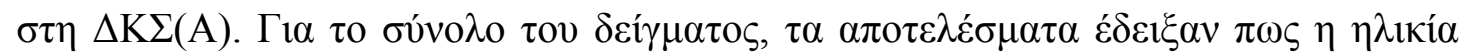




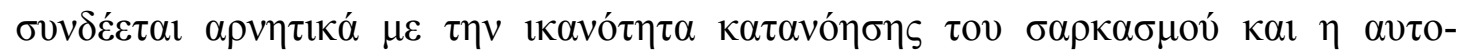

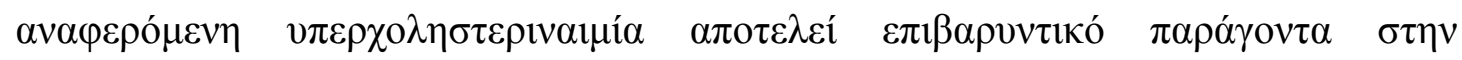
$\pi \alpha \rho \alpha \pi \alpha ́ v \omega \sigma \chi \varepsilon ́ \sigma \eta$.

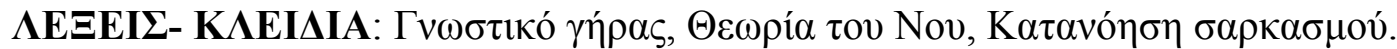




\title{
The role of age in social cognition: Comparison of young, middle- aged, and older adults in sarcasm understanding
}

\author{
Antonia Chatzieffraimidou, Despina Moraitou, Georgia Papantoniou, \\ E. Nazlidou, \& Krystallia Pantsiou
}

\begin{abstract}
The present study aimed at investigating the association between age and social cognition. The sample consisted of 72 persons between 20 and 82 years of age, equally distributed to three age groups, the "Young Adults" $(\mathrm{M}=25$ years, $S D=3.7$ years $)$, the "Middle-aged Adults" $(\mathrm{M}=51.2$ years, $S D=7.5$ years $)$ and the "Older Adults" $(\mathrm{M}=72.1$ years, $S D=4.9$ years $)$. The three groups were matched according to gender and educational level. The participants were examined with the test "Social Inference (Minimal) - SI(M): Part 2 of the TASIT (McDonald, Flanagan, Rollins, \& Kinch, 2003)", which examines whether the participant understands sarcasm and is able to differentiate sarcasm from sincerity. Moreover, the Mini Mental State Examination - MMSE (MMSE, Folstein, Folstein, \& McHugh, 1975, Greek adaptation: Fountoulakis, Tsolaki, Chatzi \& Kazis, 2000) and the Geriatric Depression Scale-15 (GDS-15, Yesavage, et al., 1982-1983, Greek adaptation: Fountoulakis, Tsolaki, Iacovides, et al., 1999) were administered to older adults. The results for older adults showed that the lower the performance on the MMSE and the higher the performance on the GDS-15, the lower the performance on the SI(M). Concerning the whole sample, the results showed that age is negatively associated with sarcasm understanding, and self-reported hypercholesterolaemia plays an aggravating role in the aforementioned relation.
\end{abstract}


KEY-WORDS: Cognitive aging, Theory of Mind, Sarcasm understanding. 


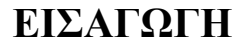

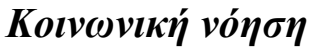

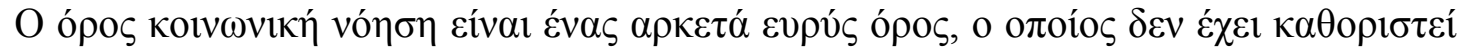

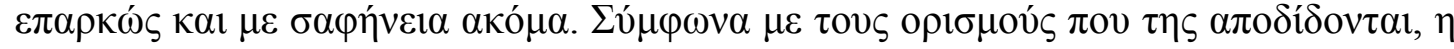

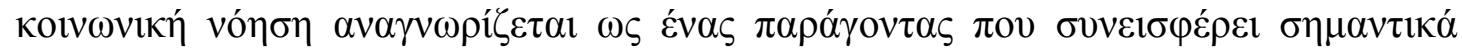

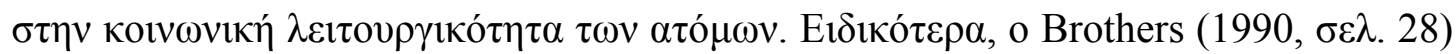

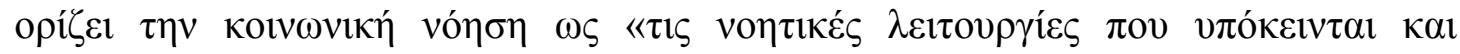

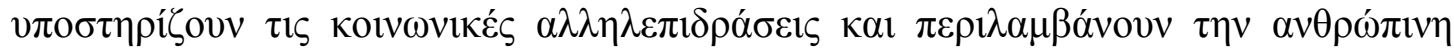

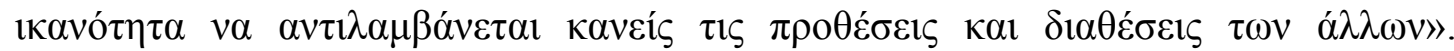

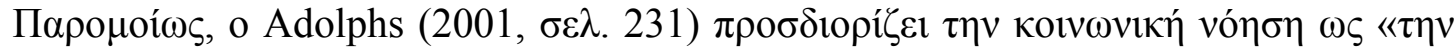

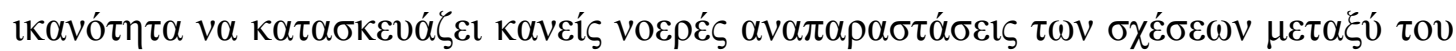

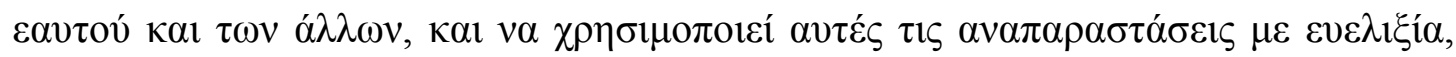

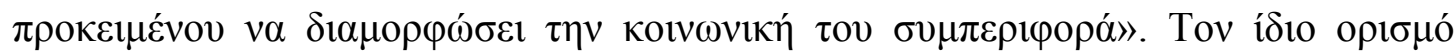

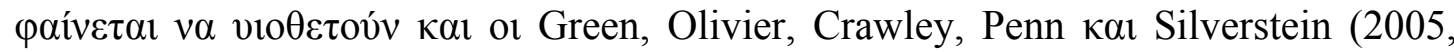

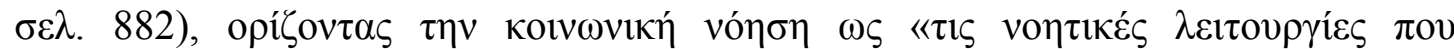

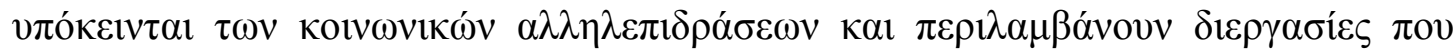

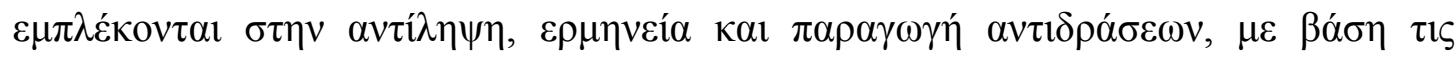

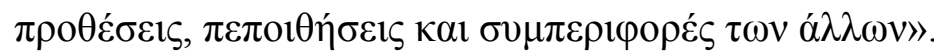

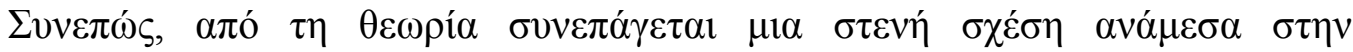

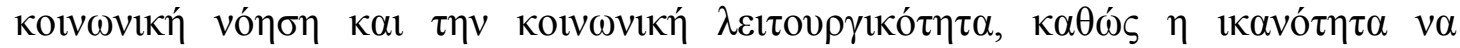

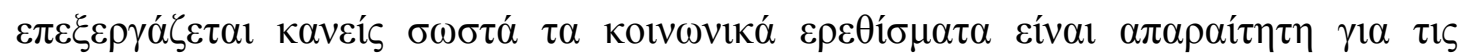

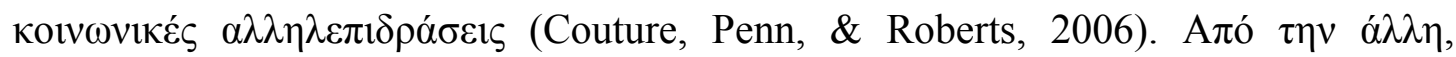

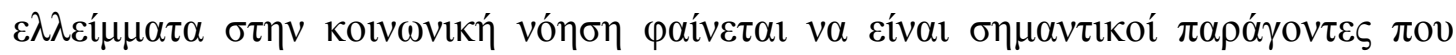

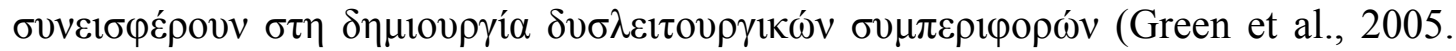




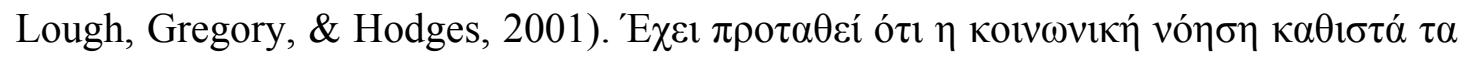

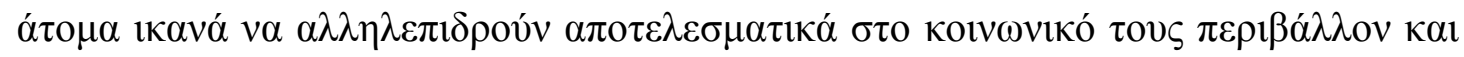

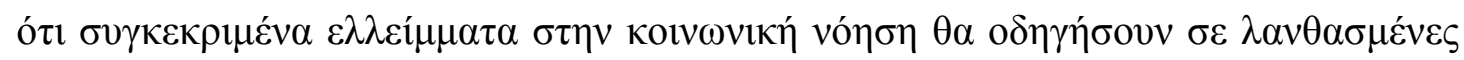

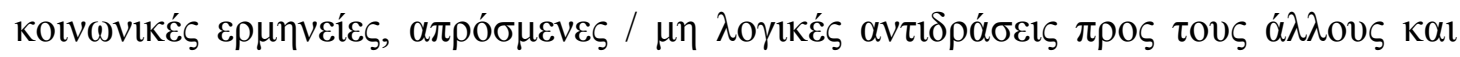

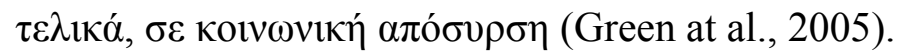

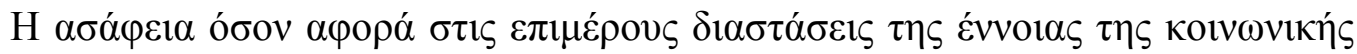

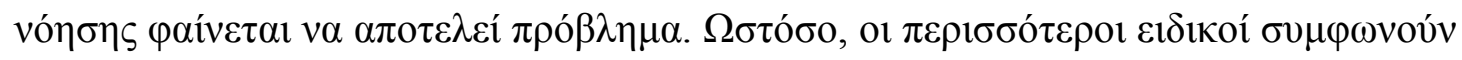

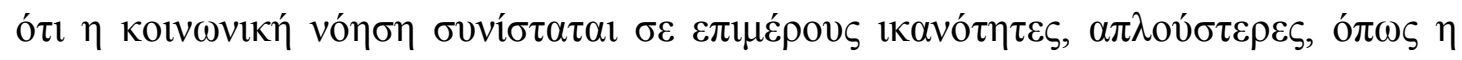

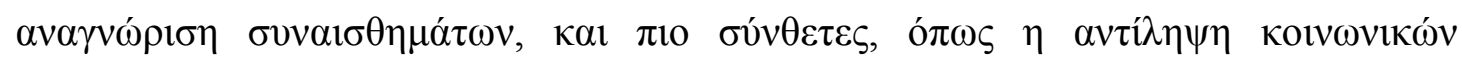

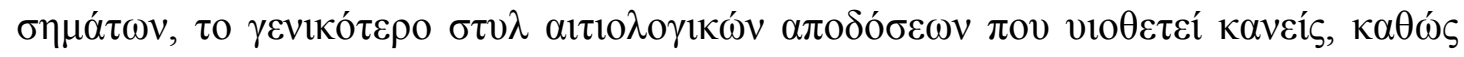

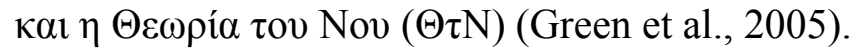

\section{$\Theta \varepsilon \omega \rho i ́ \alpha$ tov Nov $(\Theta \tau N)$}

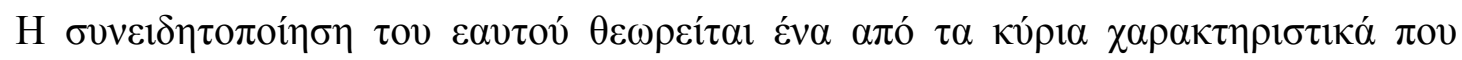

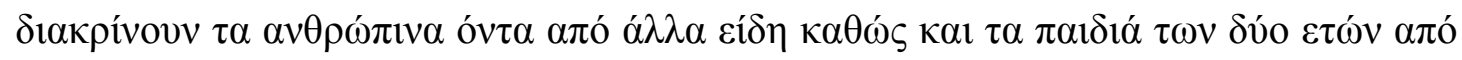

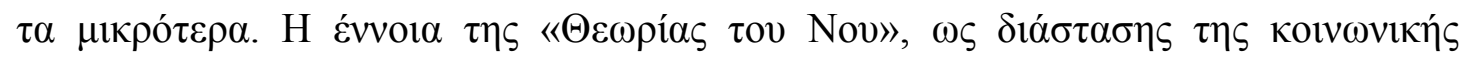

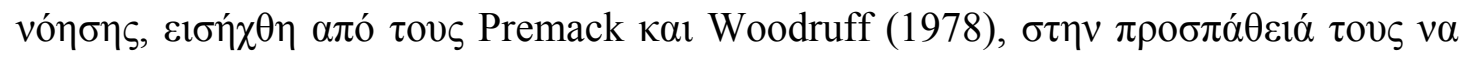

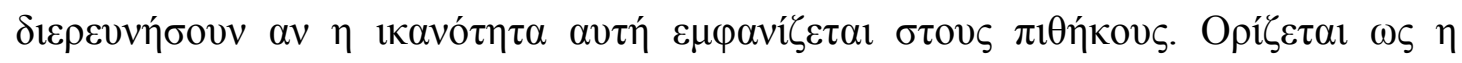

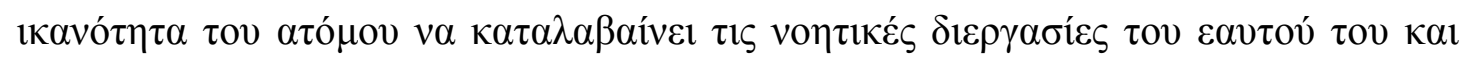

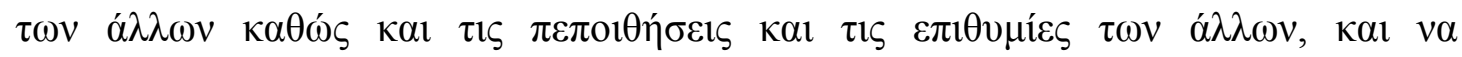

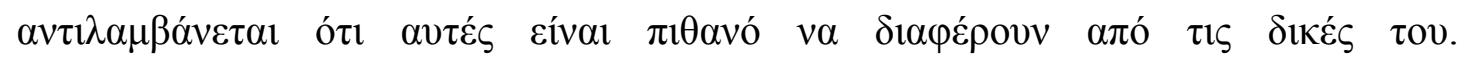

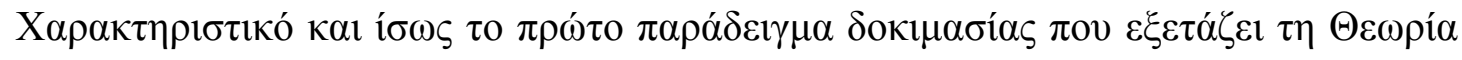

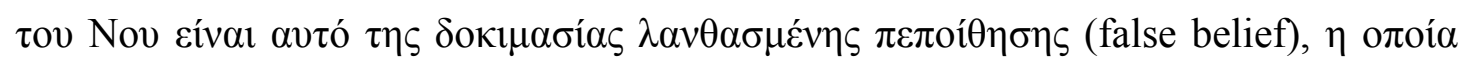

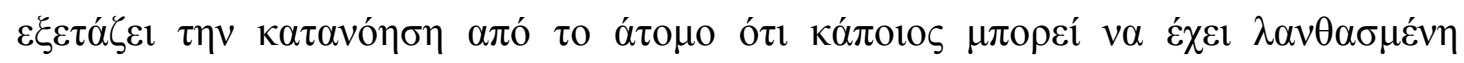

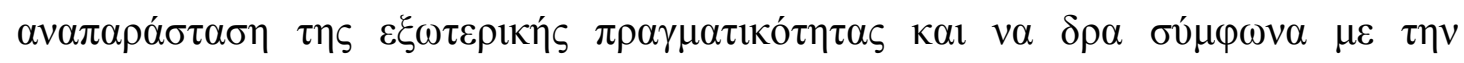




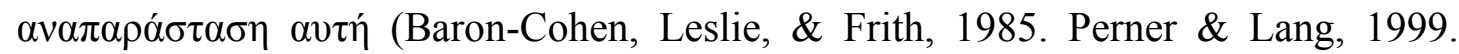
Perner \& Wimmer, 1985).

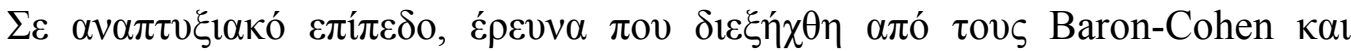

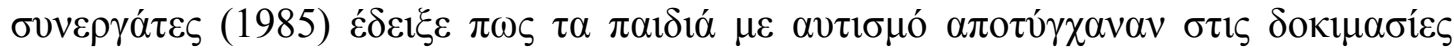

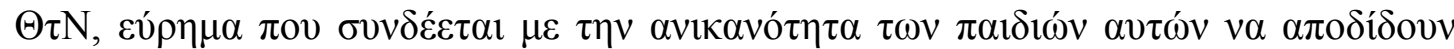

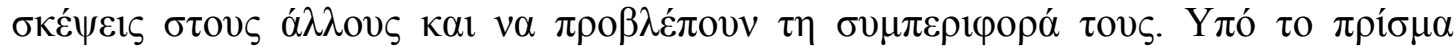

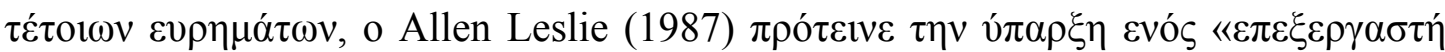

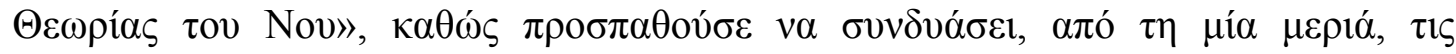

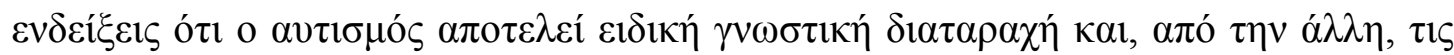

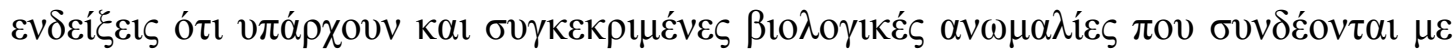

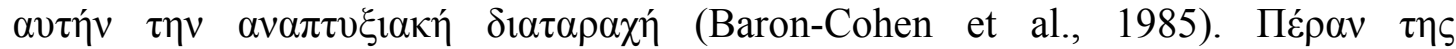

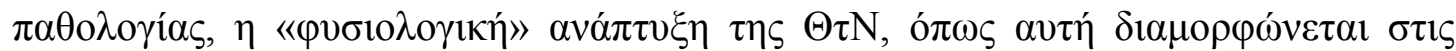

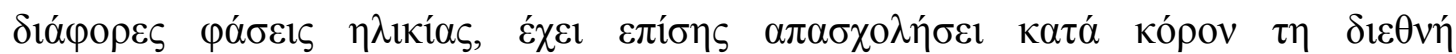

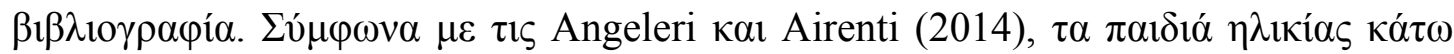

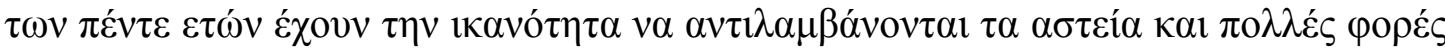

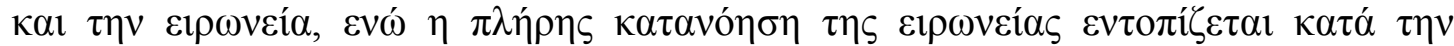

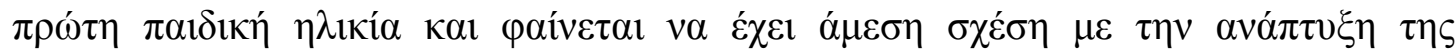

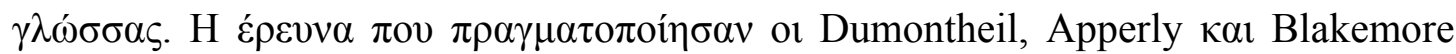

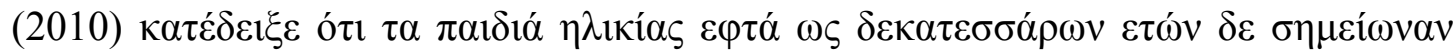

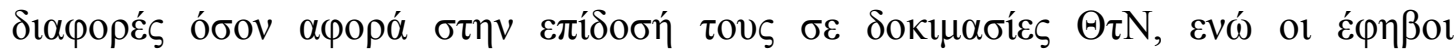

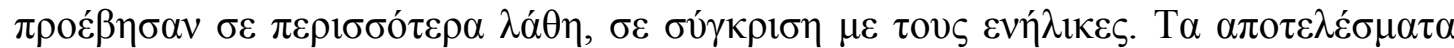

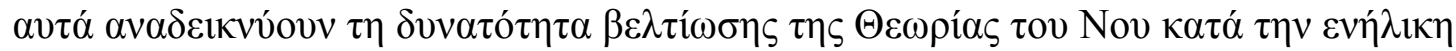
$\zeta \omega \eta ́$.

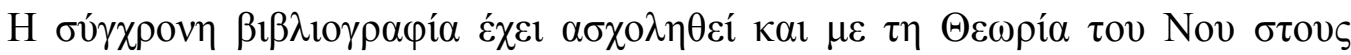

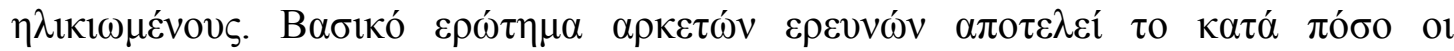




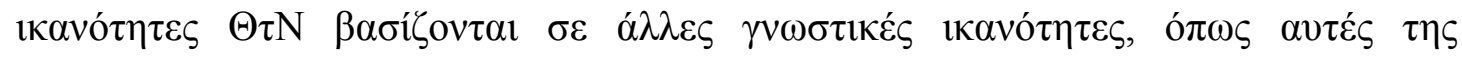

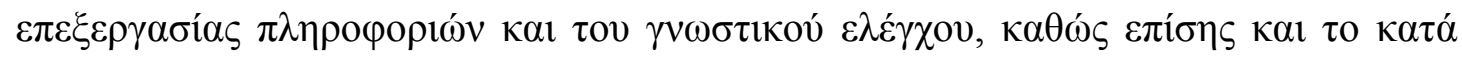

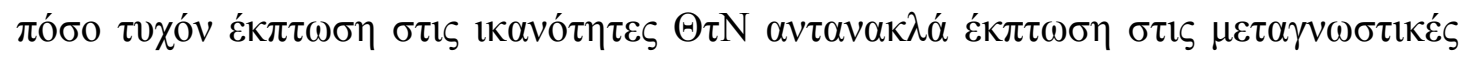

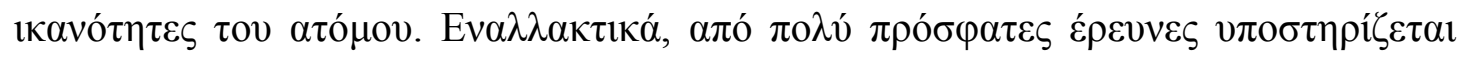

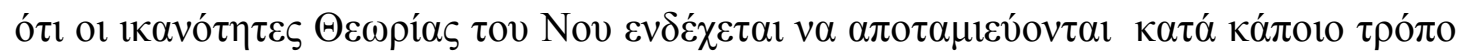

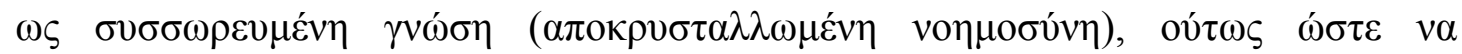

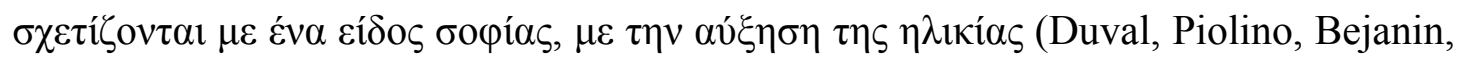
Eustache, \& Desgranges, 2011. Moran, 2013).

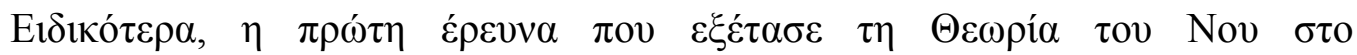

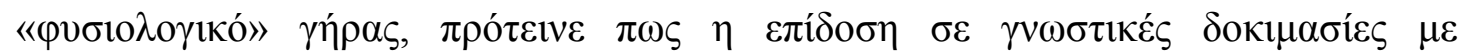

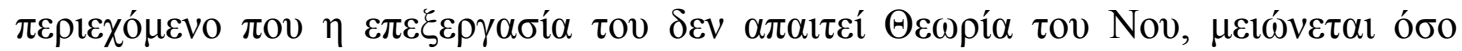

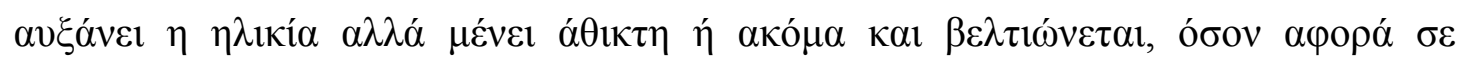

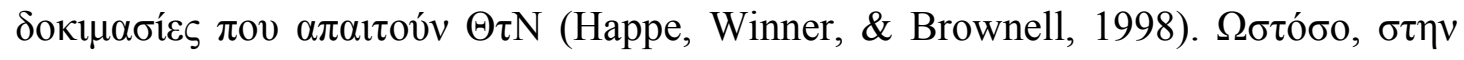

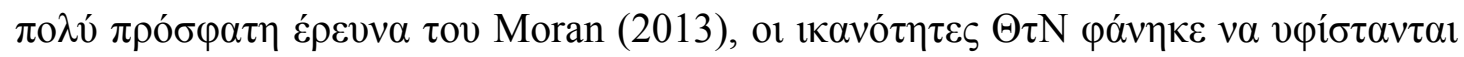

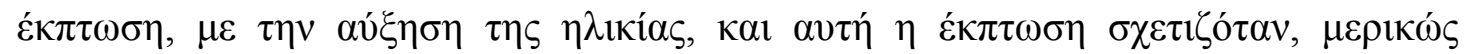

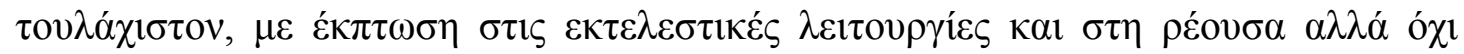
$\sigma \tau \eta \nu$ a

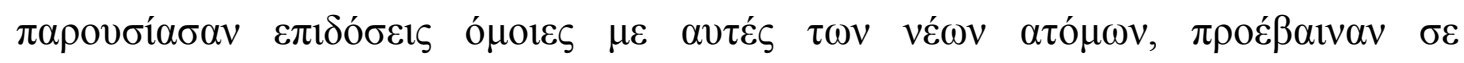

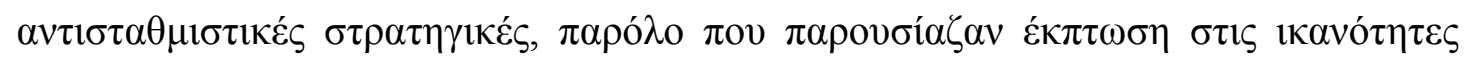

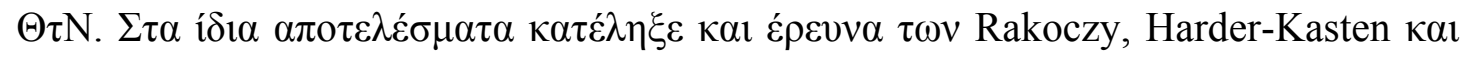

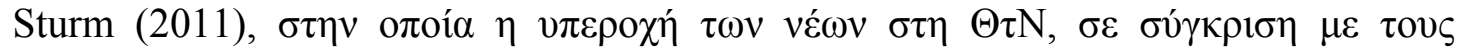

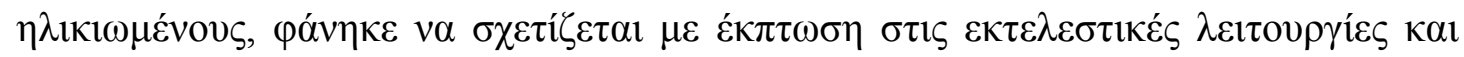

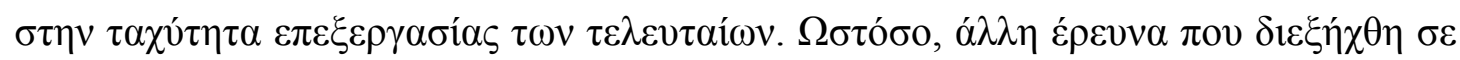

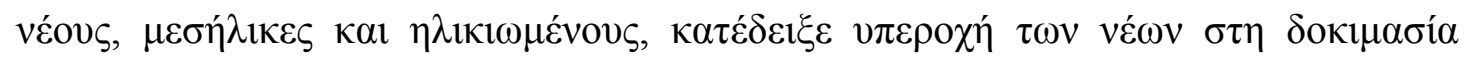

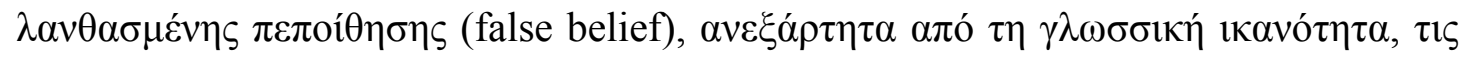




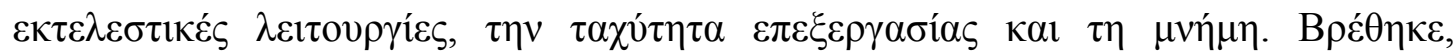

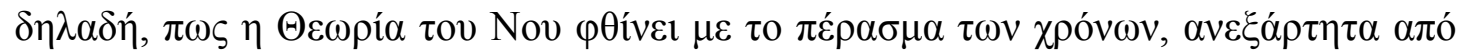

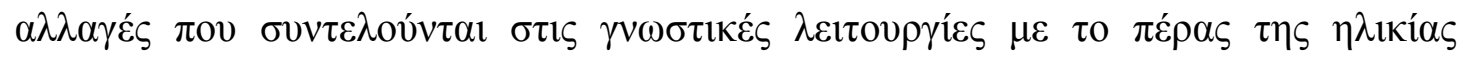

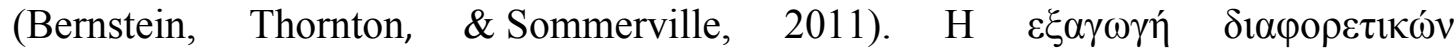

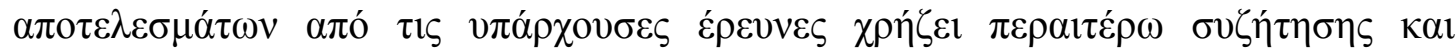

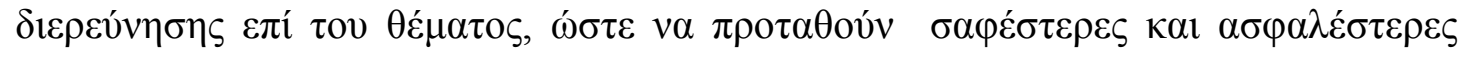

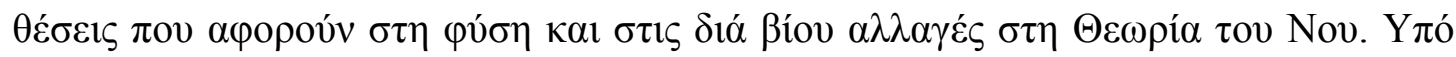

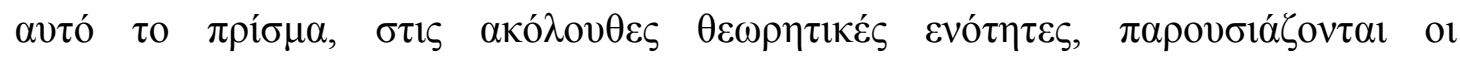

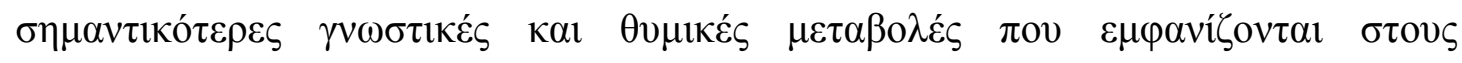

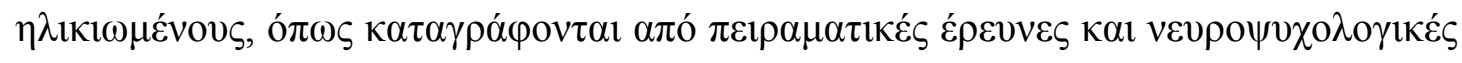
$\mu \varepsilon \lambda \dot{\varepsilon} \tau \varepsilon \zeta$.

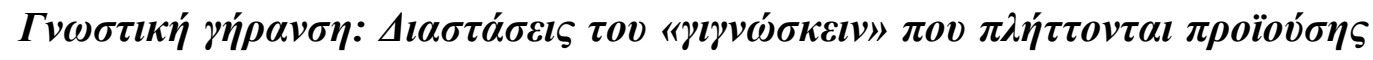

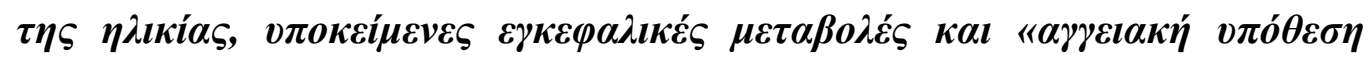

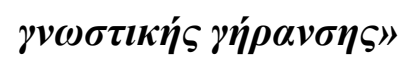

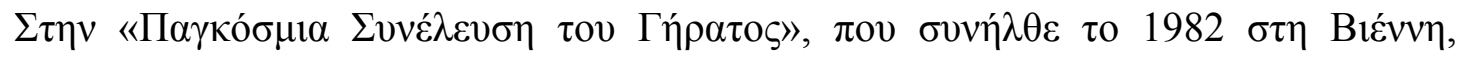

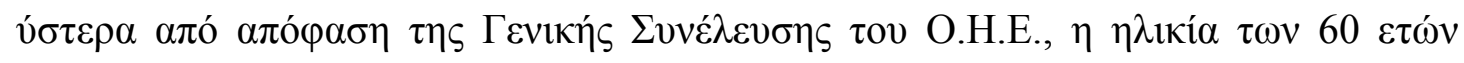

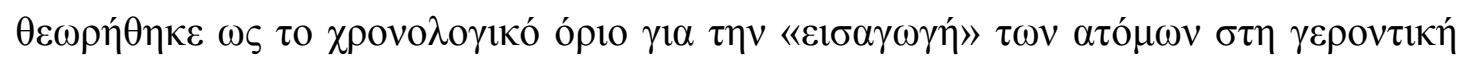

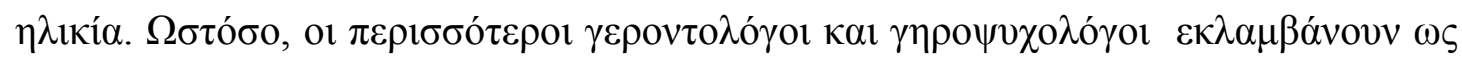

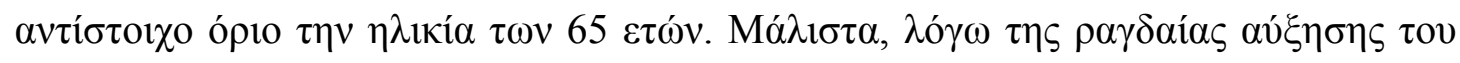

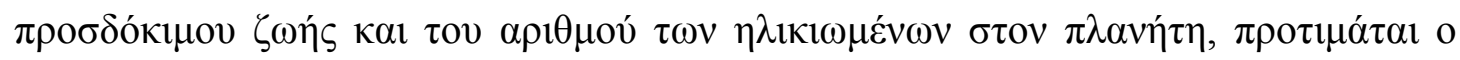

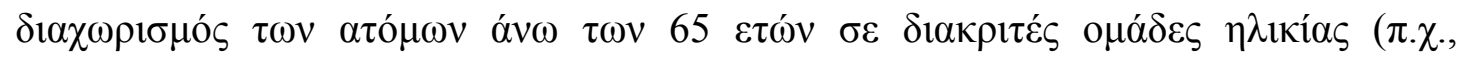

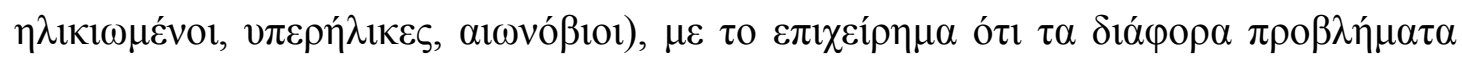

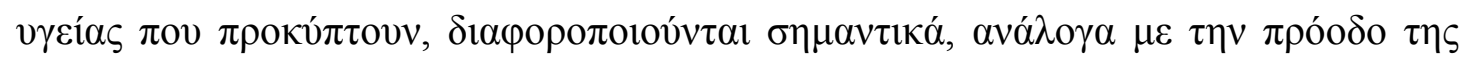




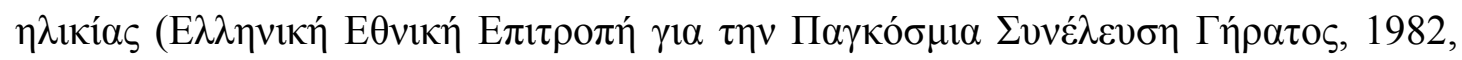

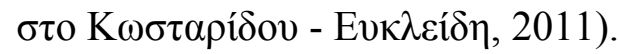

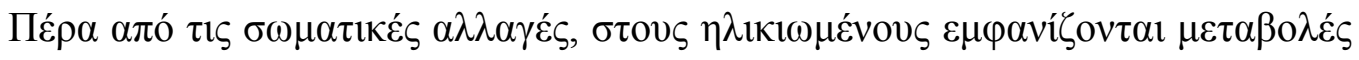

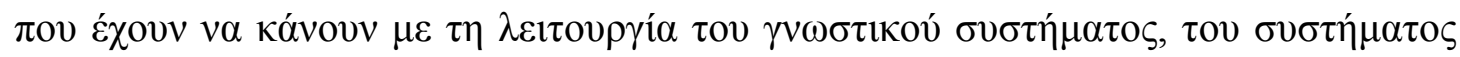

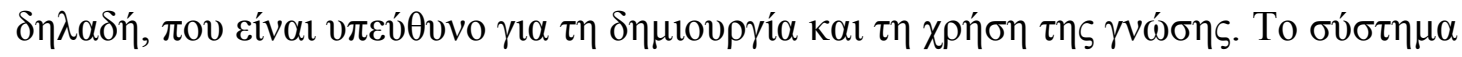

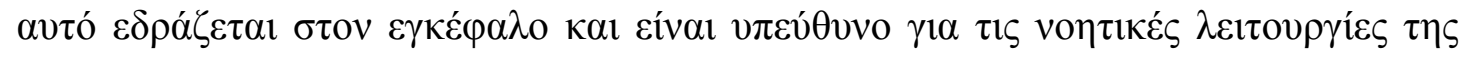

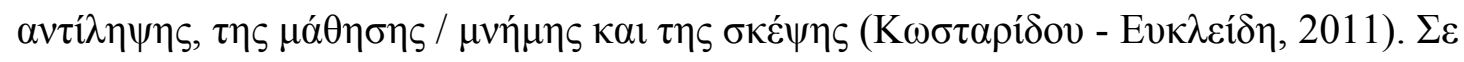

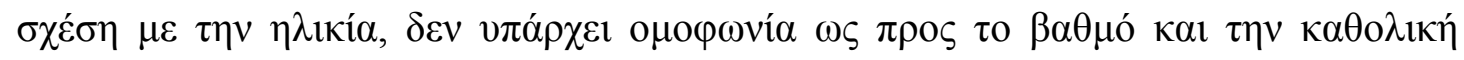

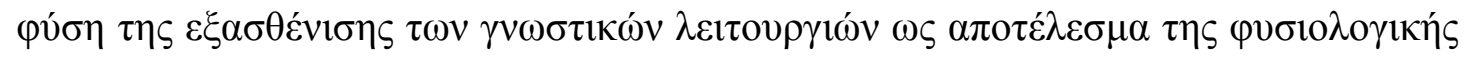

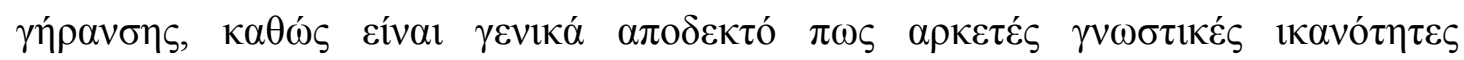

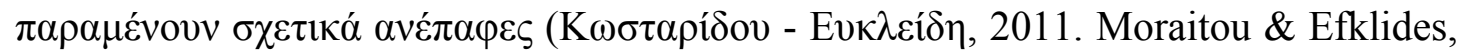

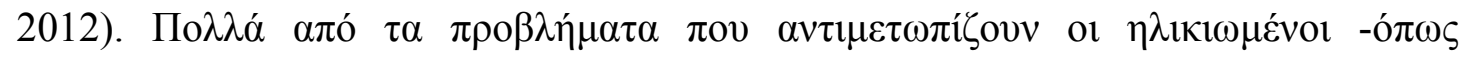

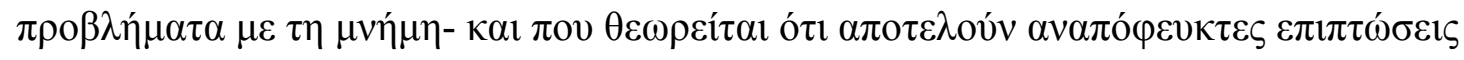

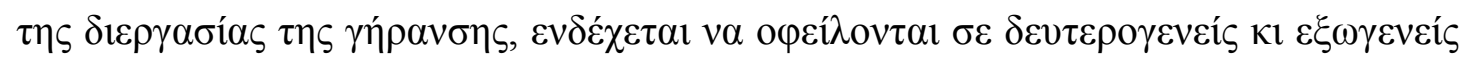

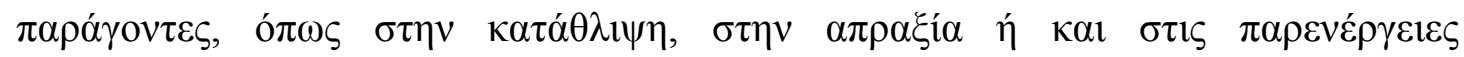

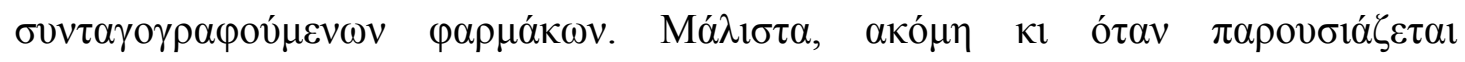

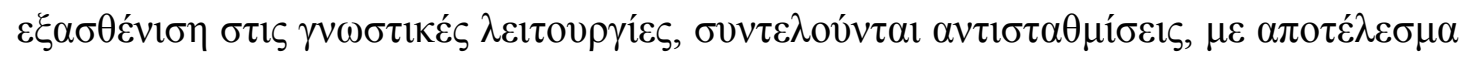

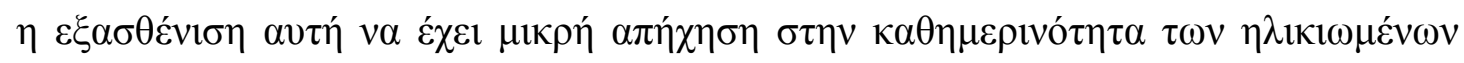
$\alpha \tau$ ó $\mu \omega v$ (Salthouse, 2004).

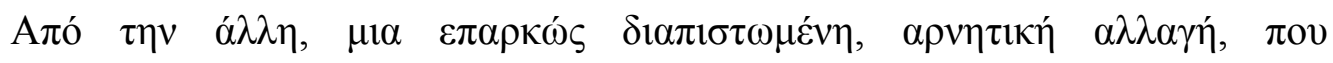

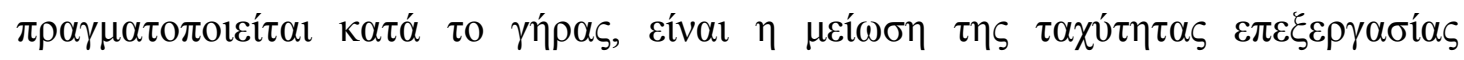

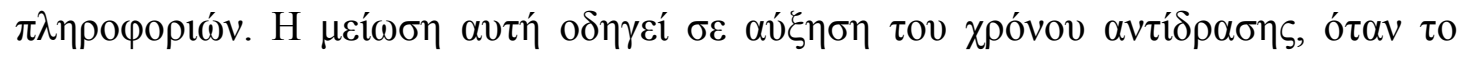

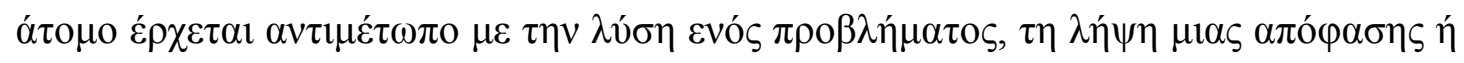

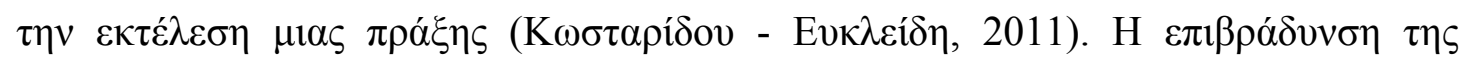

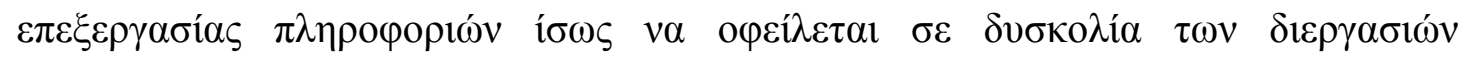




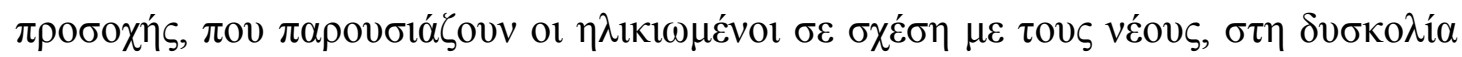

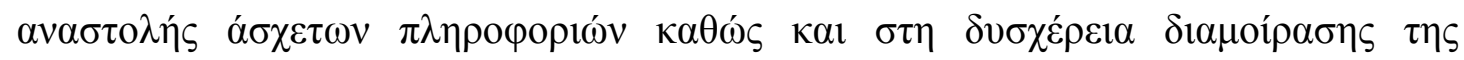

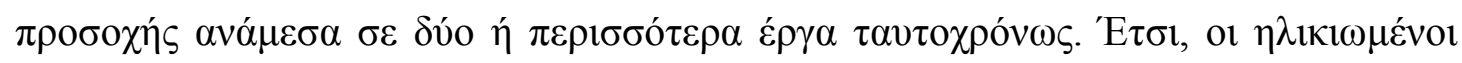

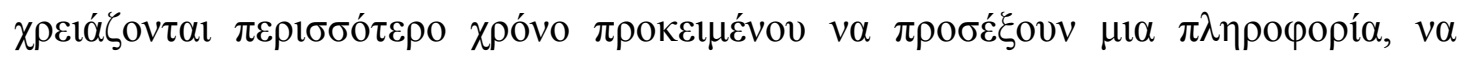

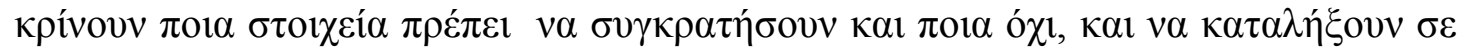
$\mu 1 \alpha \alpha \pi$ ó$\varphi \alpha \sigma \eta$.

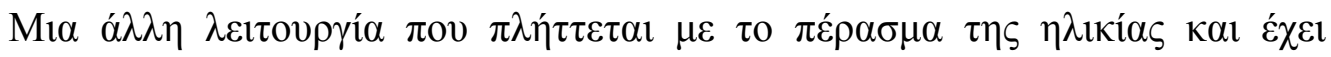

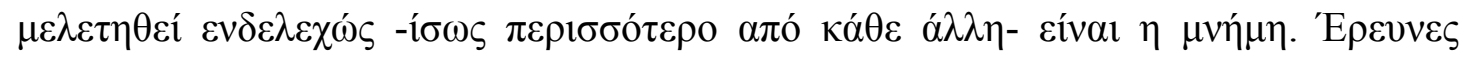

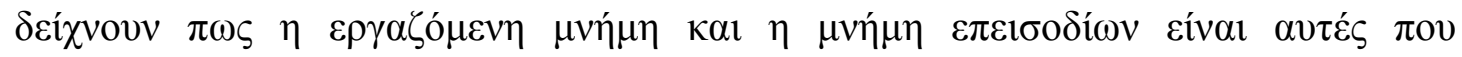

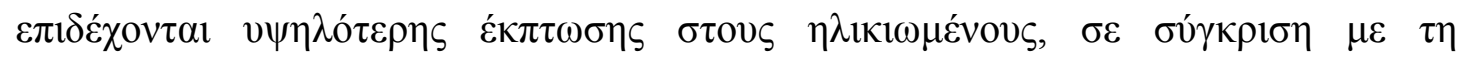

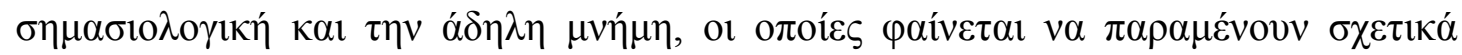

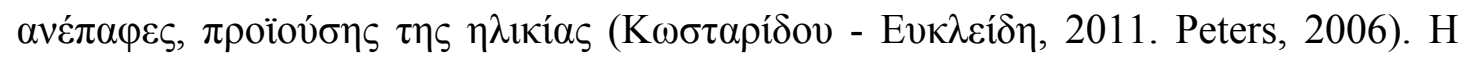

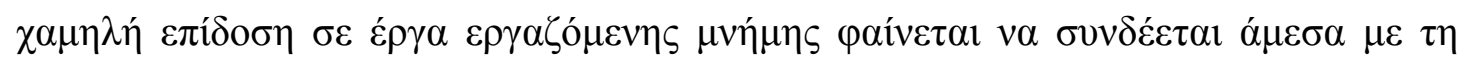

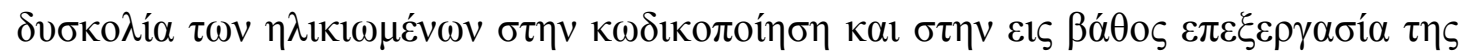

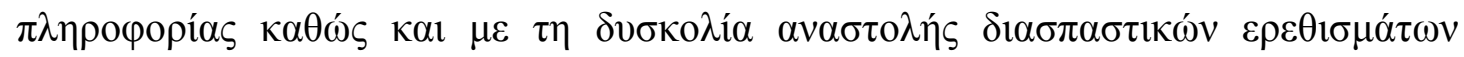

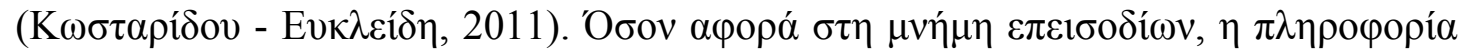

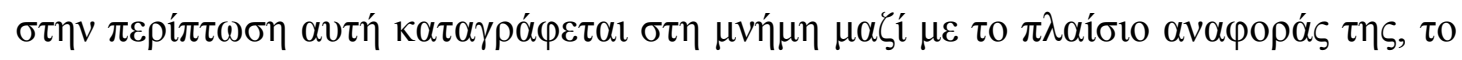

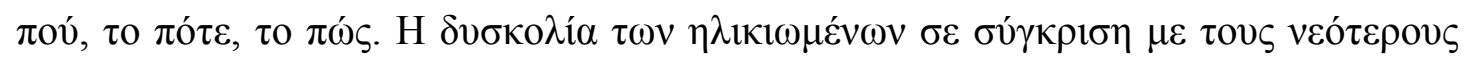

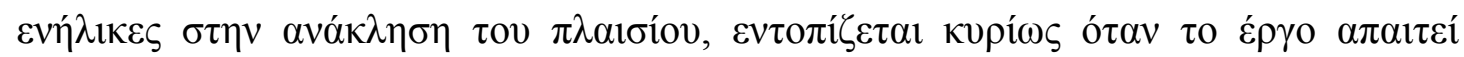

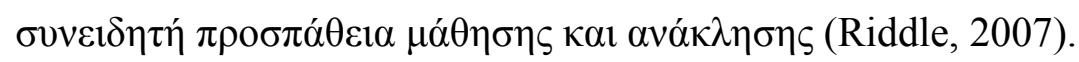

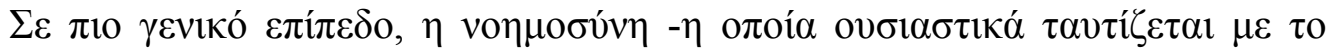

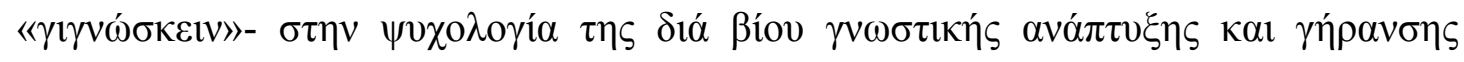

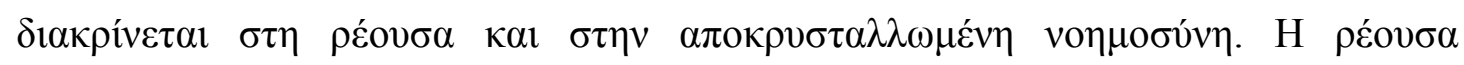

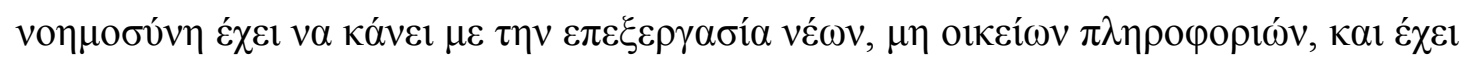

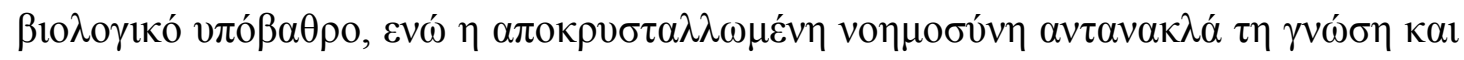




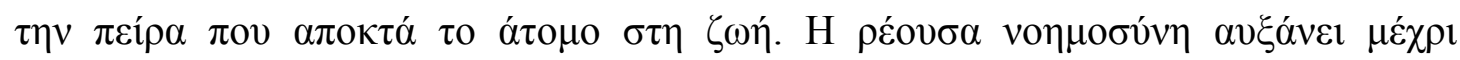

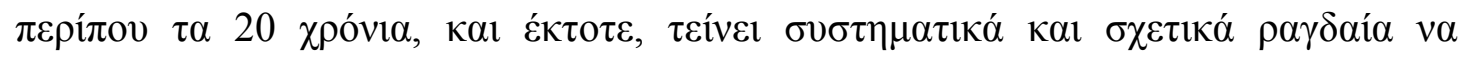

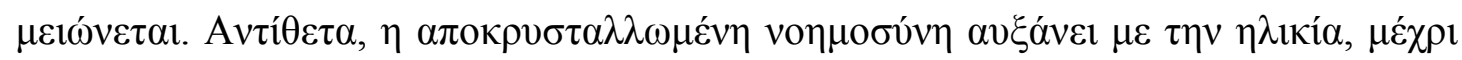

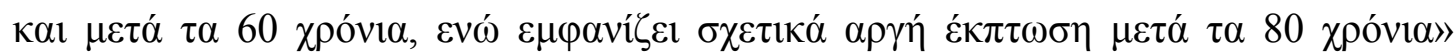

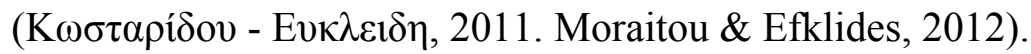

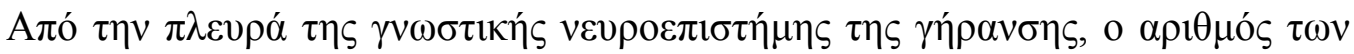

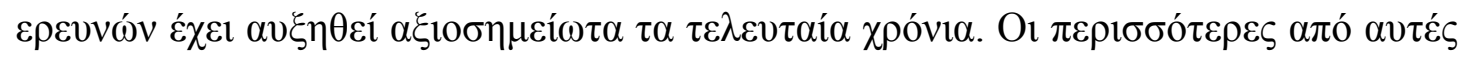

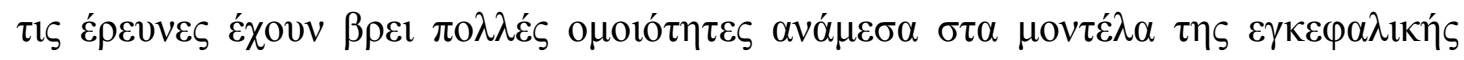

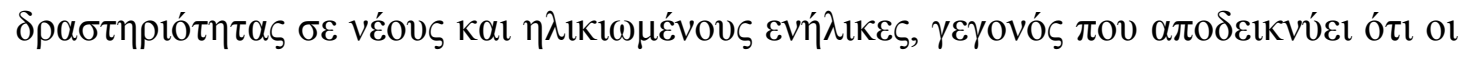

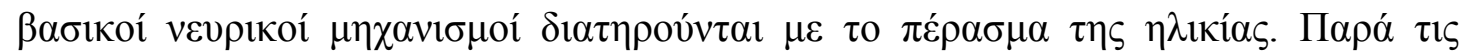

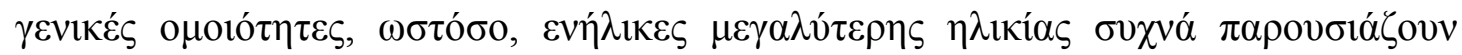

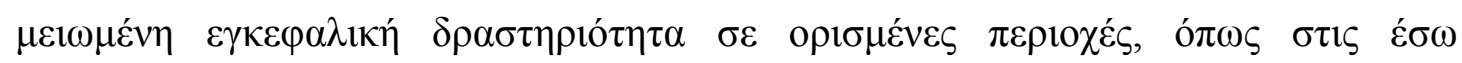

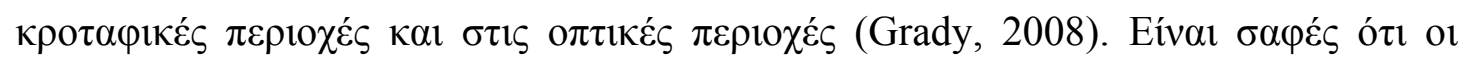

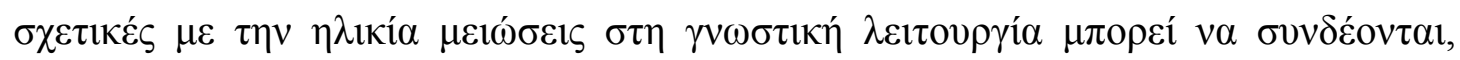

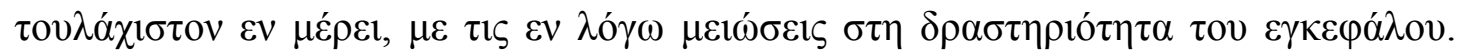

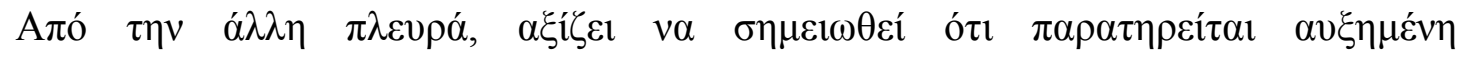

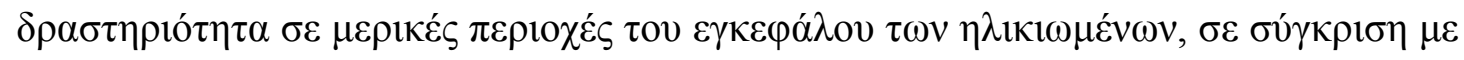

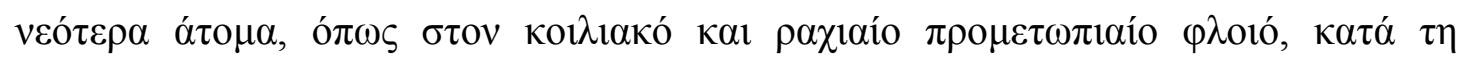

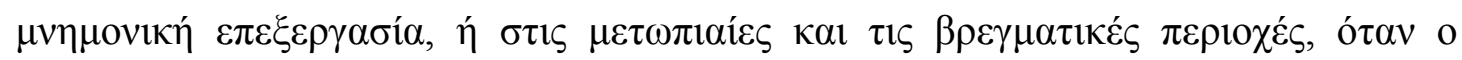

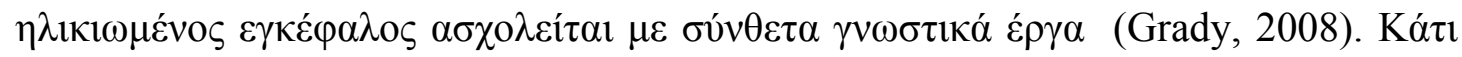

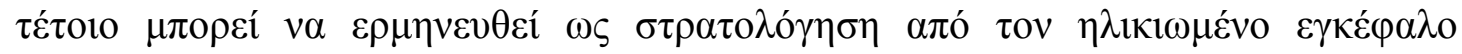

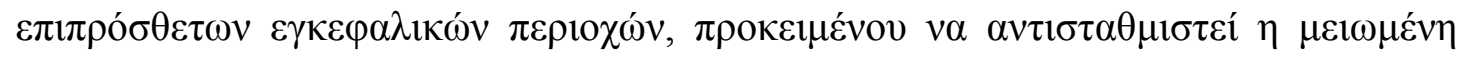

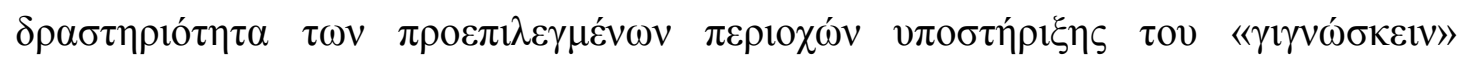
(Grady, 2008. Kryla-Lighthall \& Mather, 2009). 


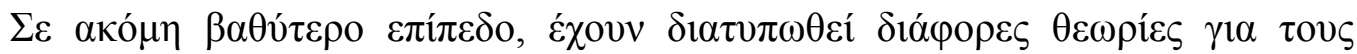

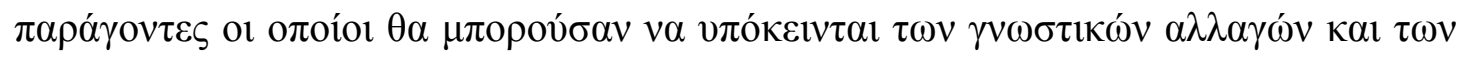

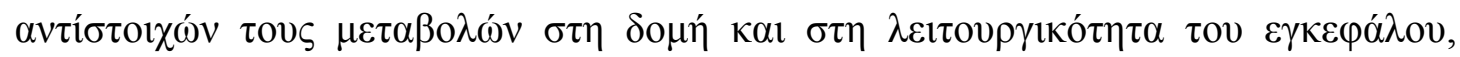

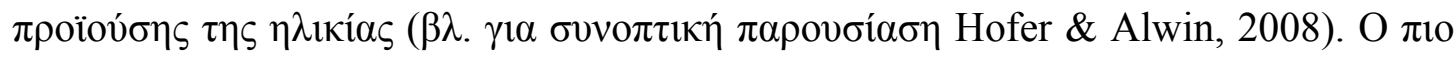

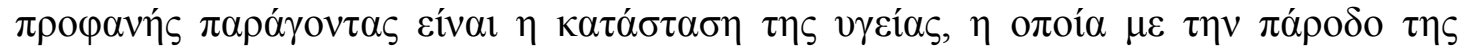

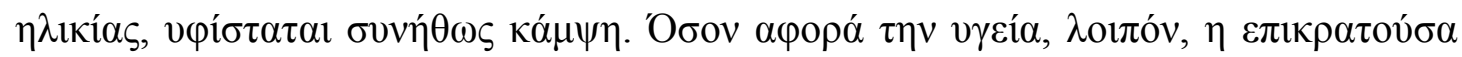

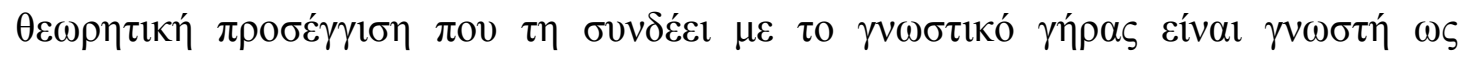

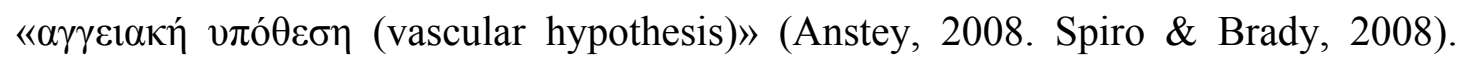

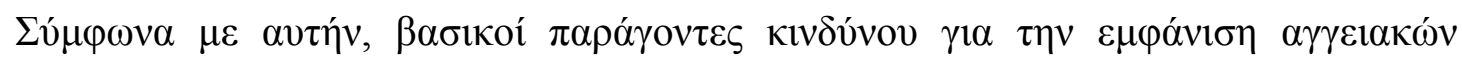

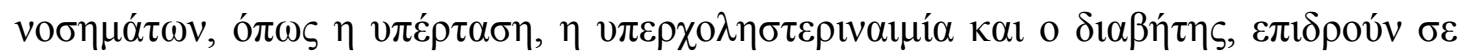

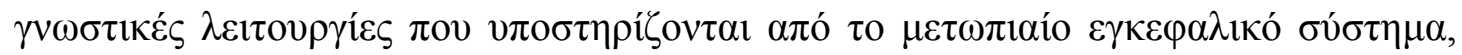
ó $\pi \omega \varsigma, \gamma 1 \alpha \pi \alpha \rho \alpha ́ \delta \varepsilon 1 \gamma \mu \alpha, \eta \varepsilon \rho \gamma \alpha \zeta o ́ \mu \varepsilon v \eta \mu \nu \eta ́ \mu \eta$ (Elias, Elias, D’Agostino, Sullivan, \&

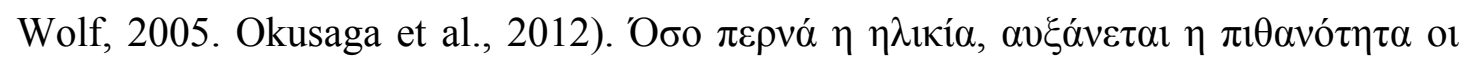

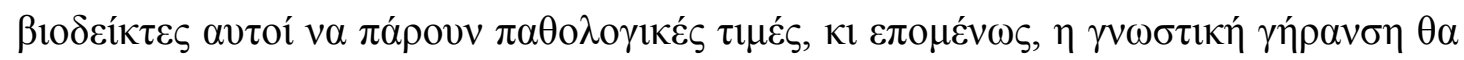
$\mu \pi$

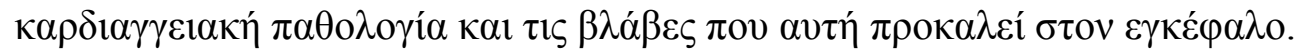

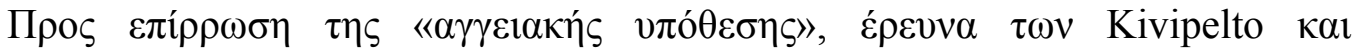

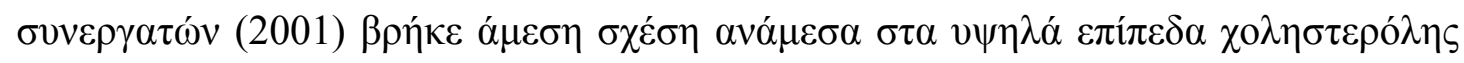

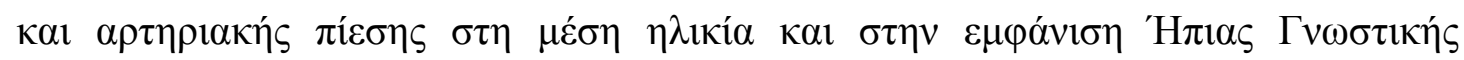

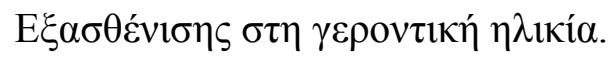

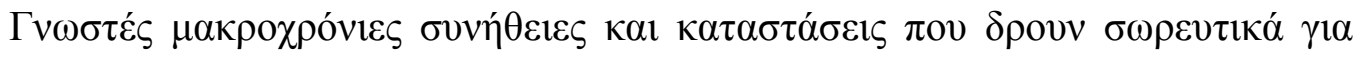

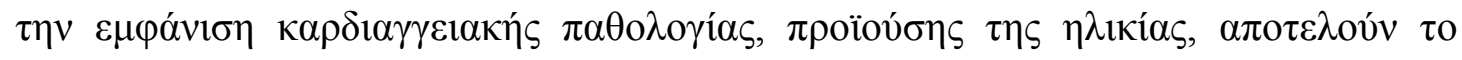

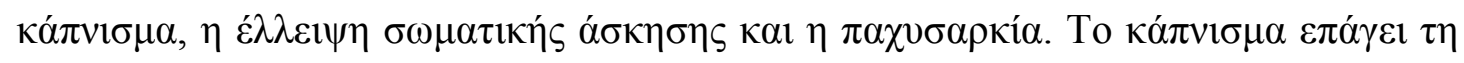

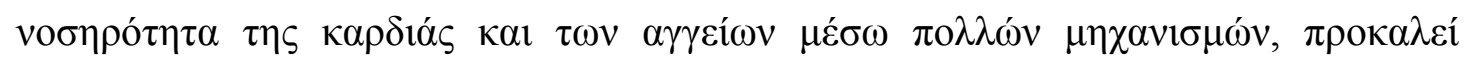

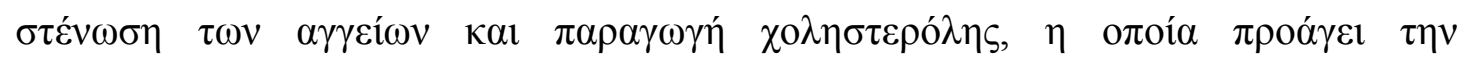




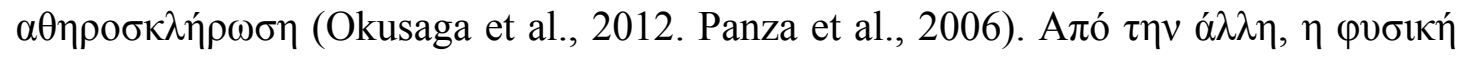

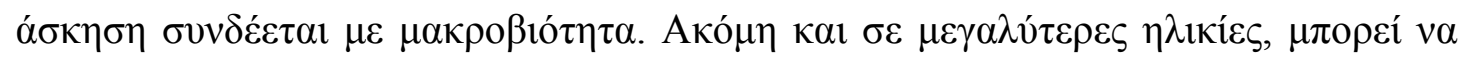

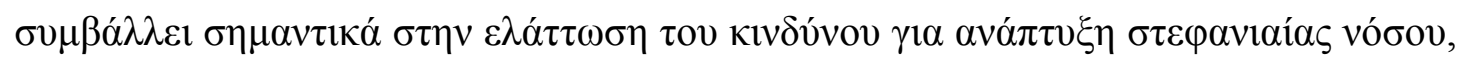

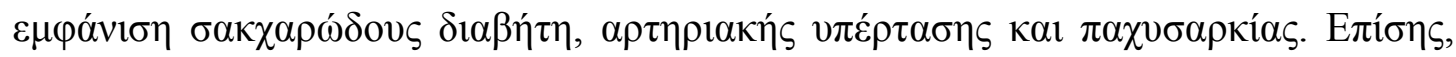

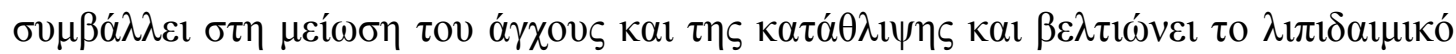

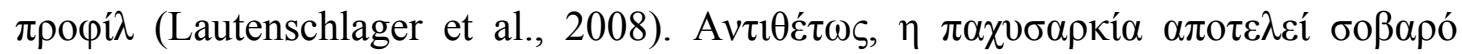

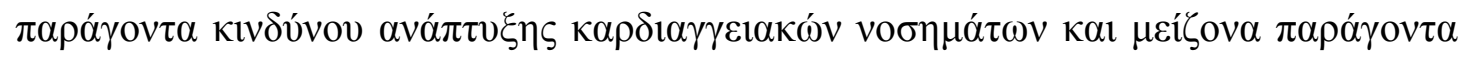

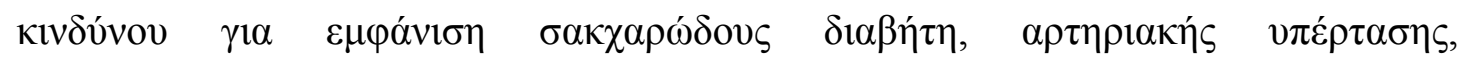

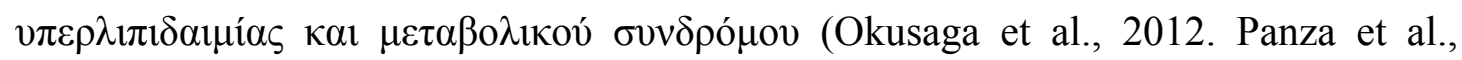
2006).

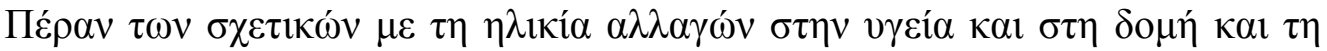

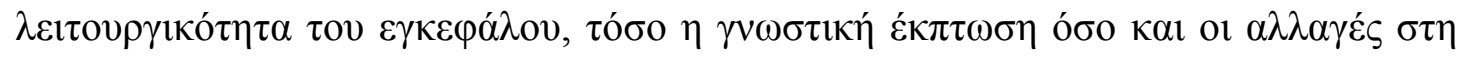

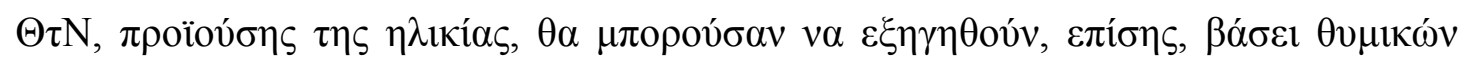

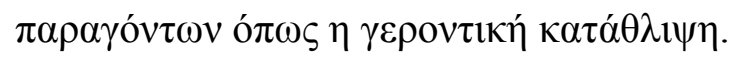

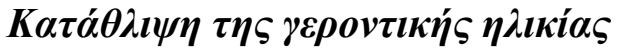

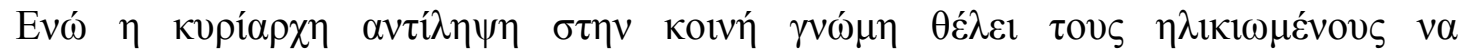

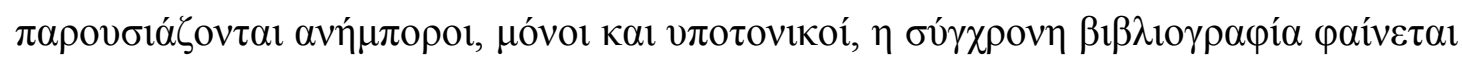

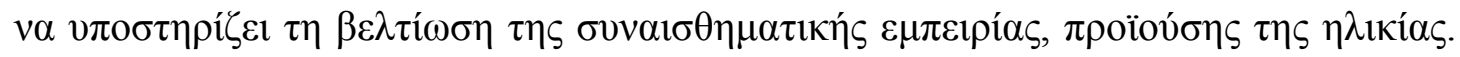

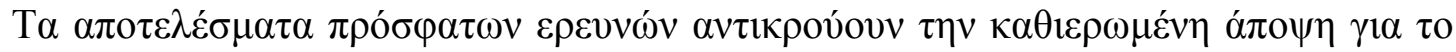

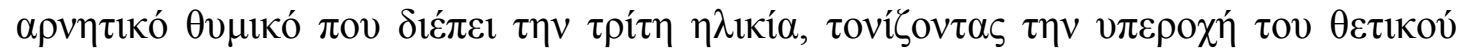

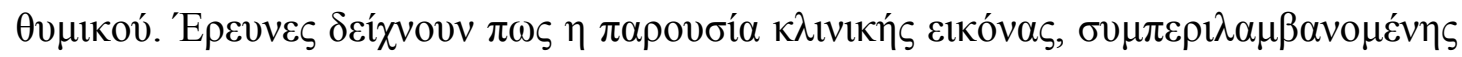

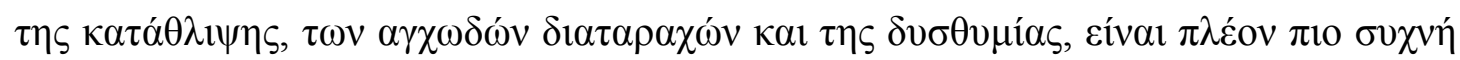

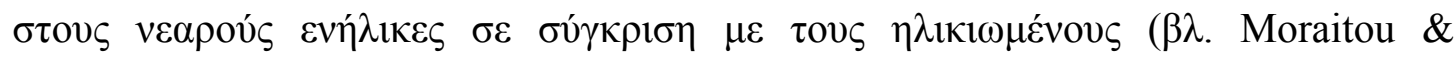
Papantoniou, 2012, Moraitou, Papantoniou, Gkinopoulos, \& Nigritinou, 2013). 


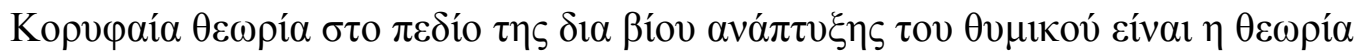

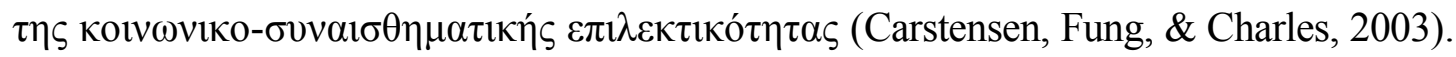

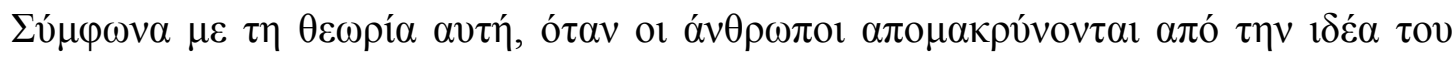

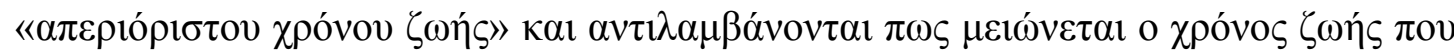

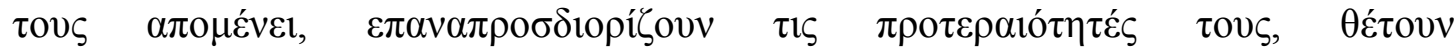

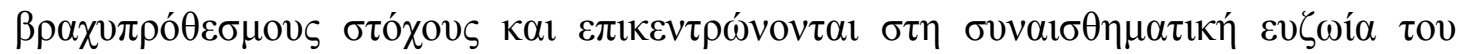

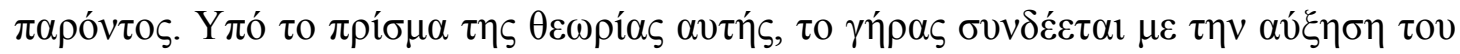

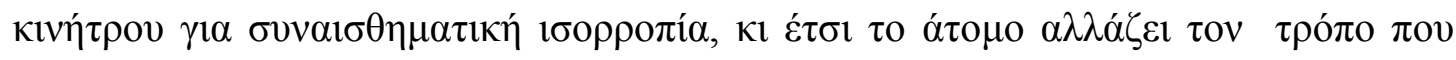

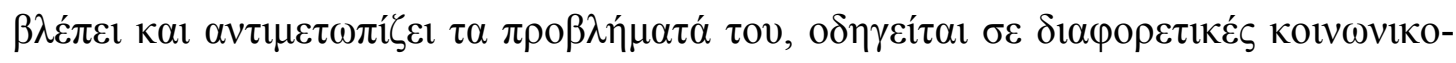

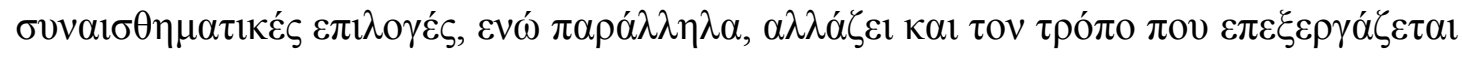

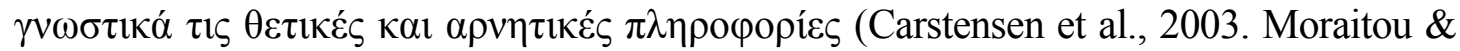
Efklides, 2007).

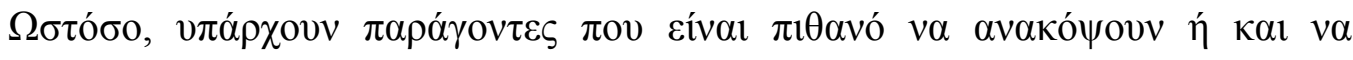

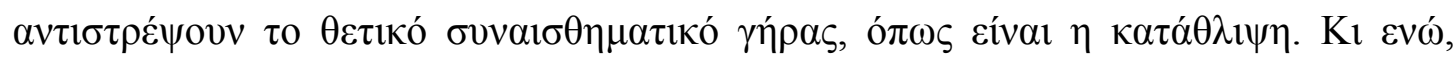

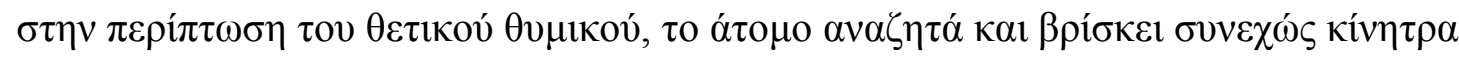

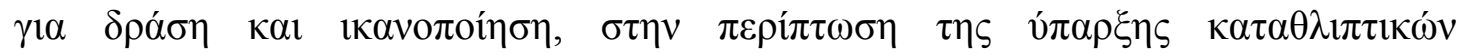

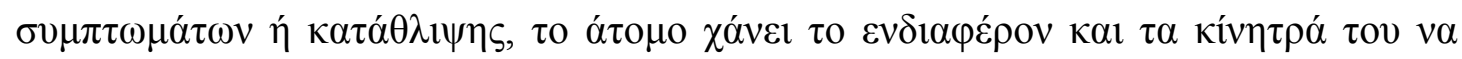

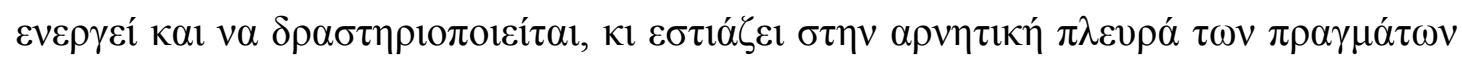

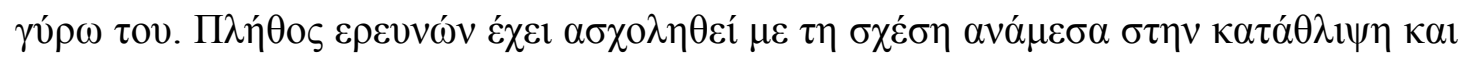

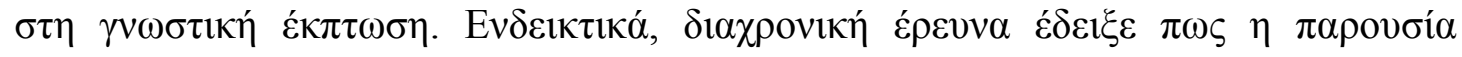

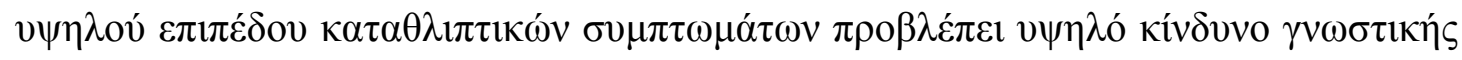

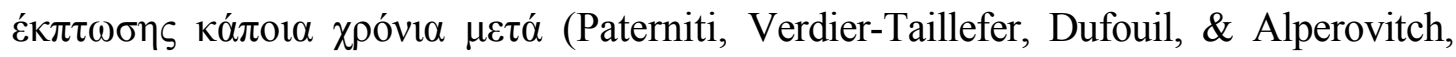

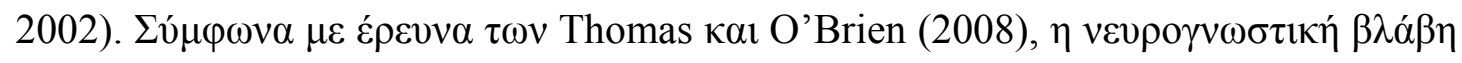

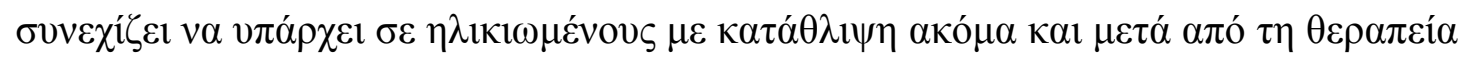

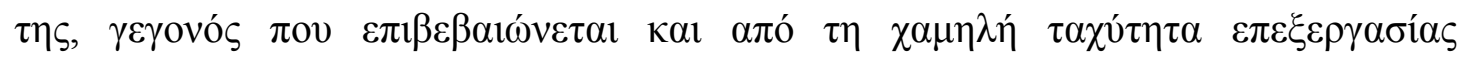




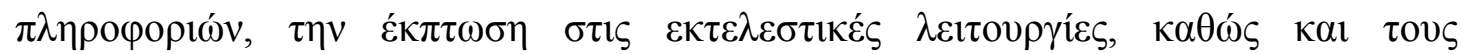

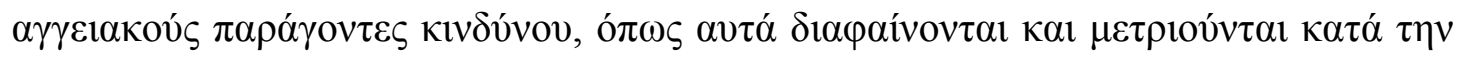

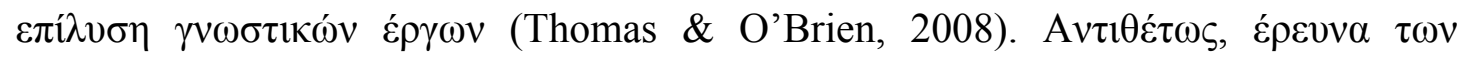

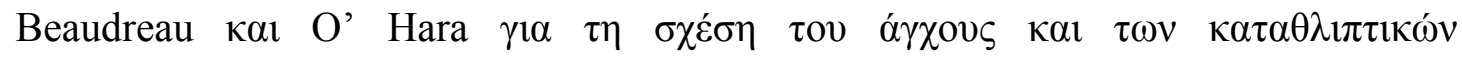

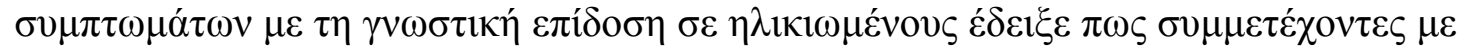

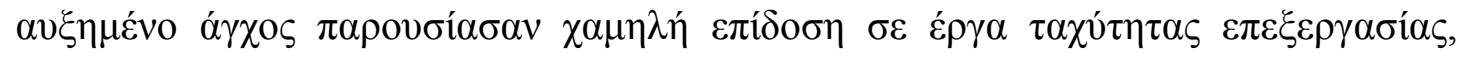

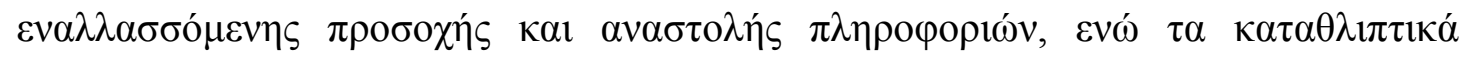

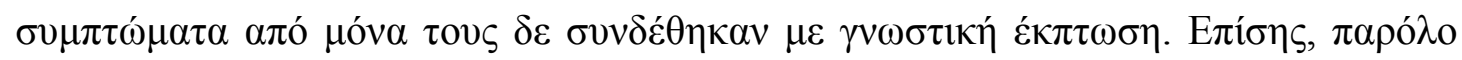

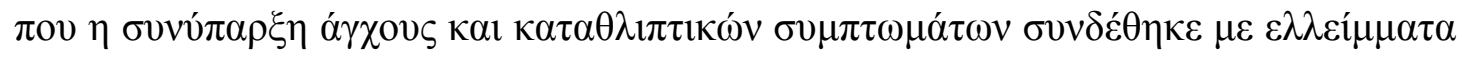

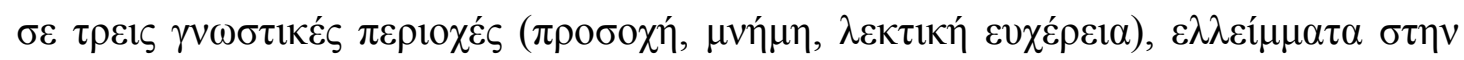

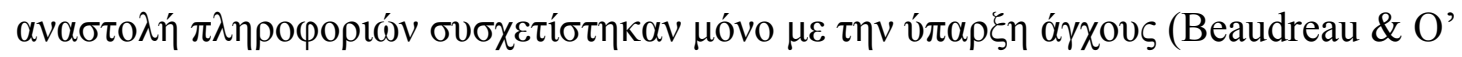

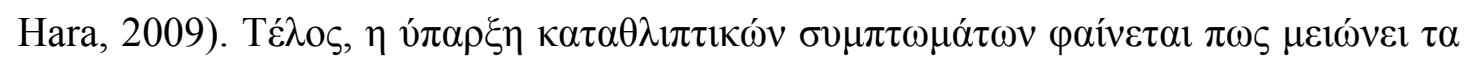

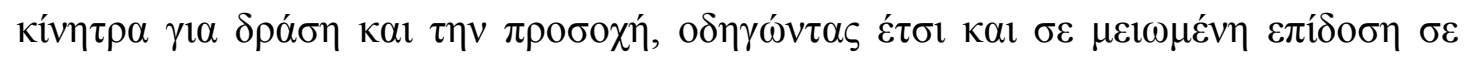

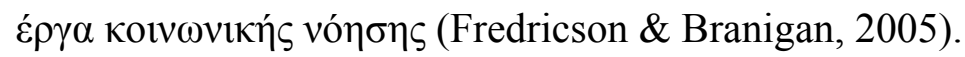

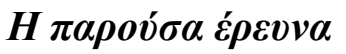

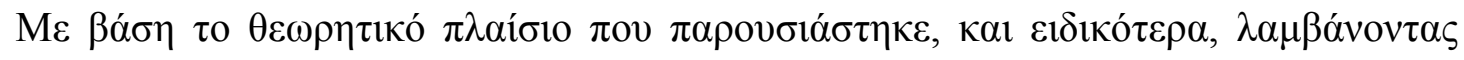

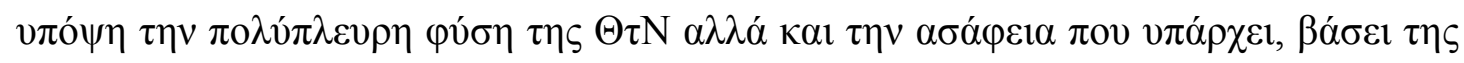

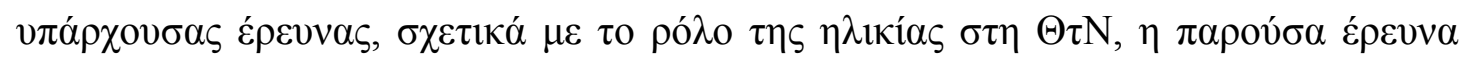

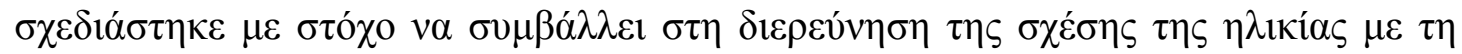

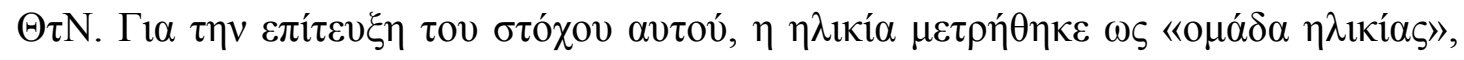

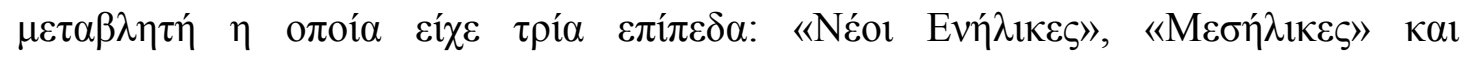

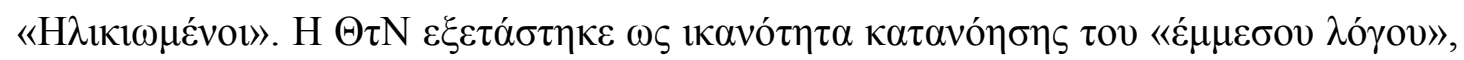

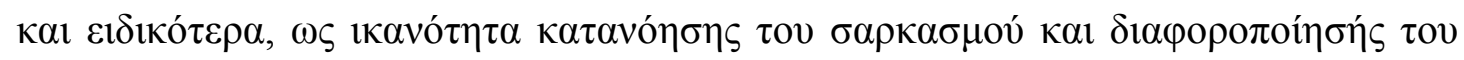

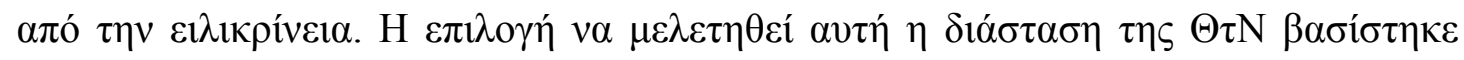




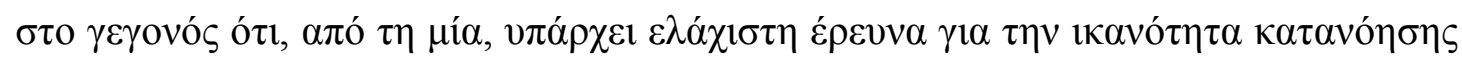

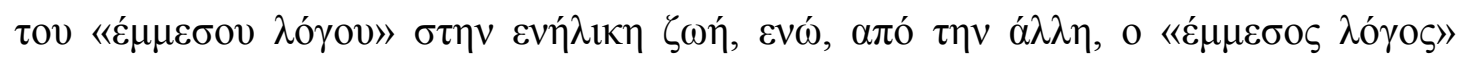

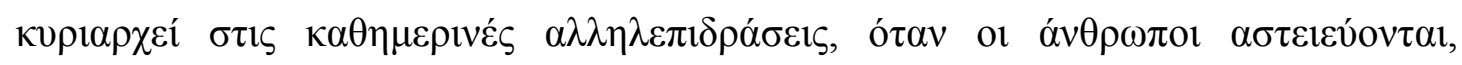

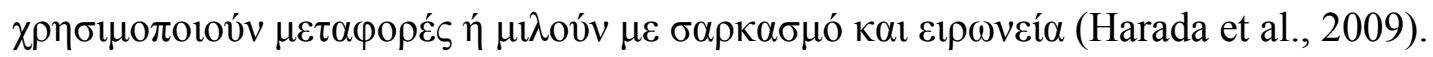

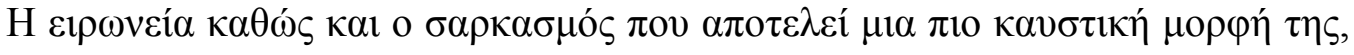

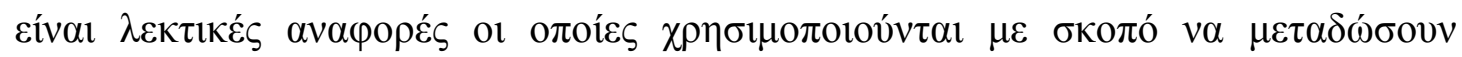

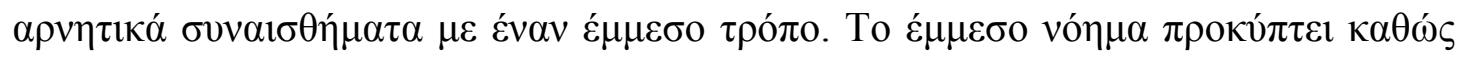

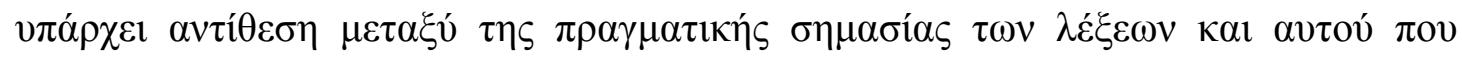

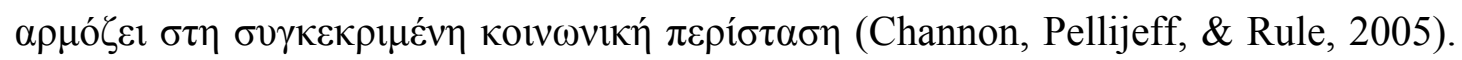

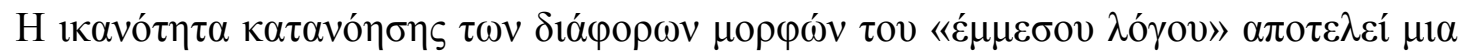

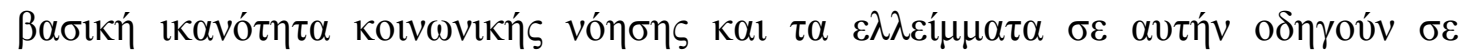

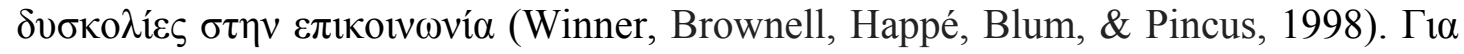

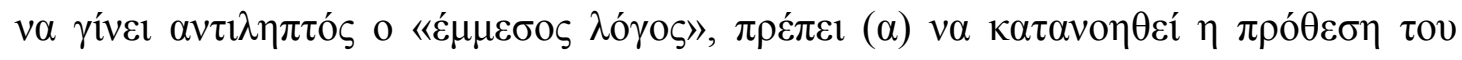

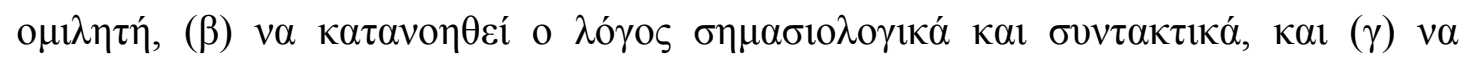

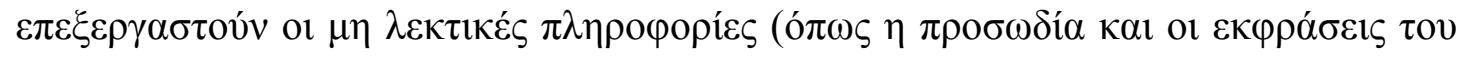

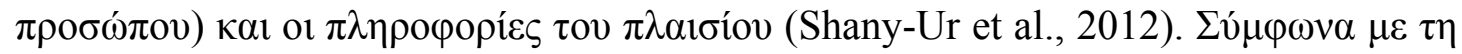

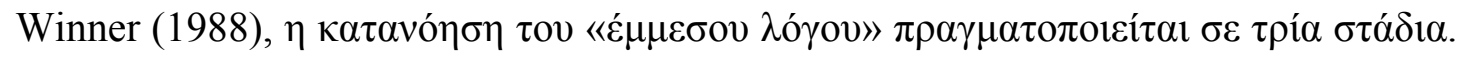

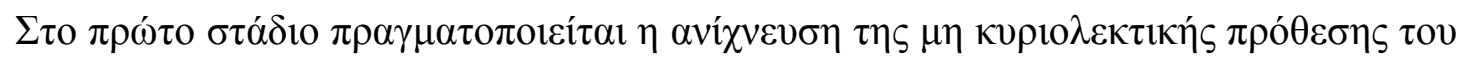

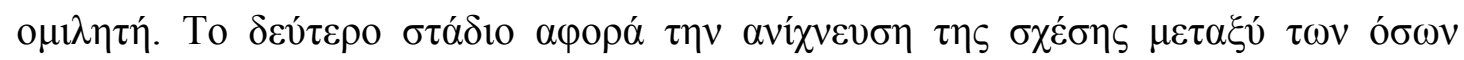

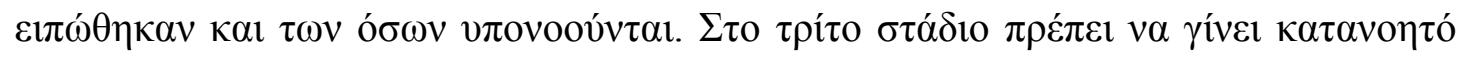

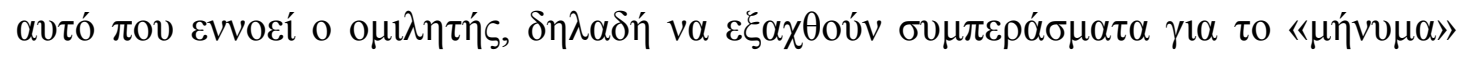

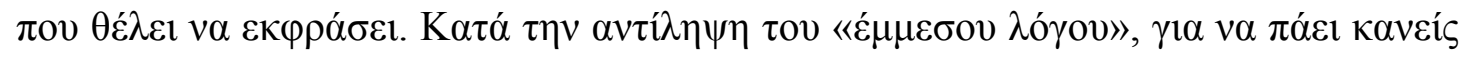

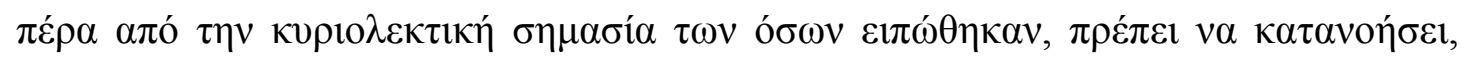

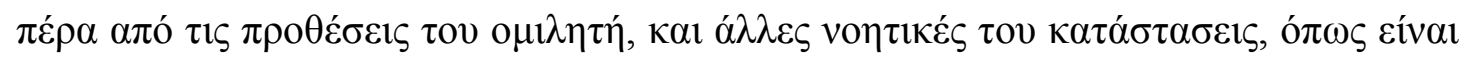

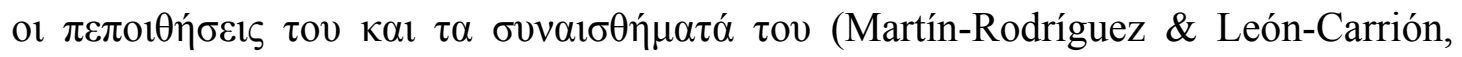




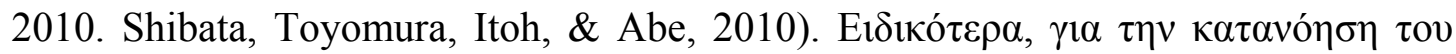

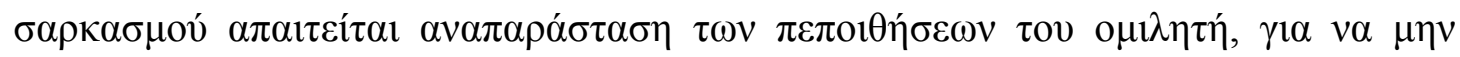

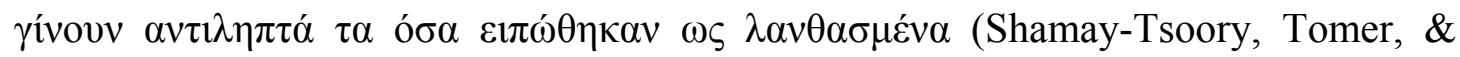

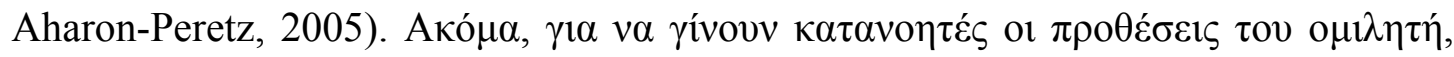

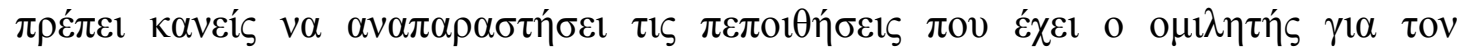
$\alpha \kappa \rho о \alpha \tau \eta ́ . ~ H ~ \sigma \omega \sigma \tau \eta ́ ~ \alpha v \alpha \pi \alpha \rho \alpha ́ \sigma \tau \alpha \sigma \eta ~ \tau \omega v ~ \pi \rho o \theta \varepsilon ́ \sigma \varepsilon \omega \nu ~ \alpha v \tau \omega ́ v ~ \sigma v v \tau \varepsilon \lambda \varepsilon i ́ ~ \sigma \tau o ~ v \alpha \mu \eta v$

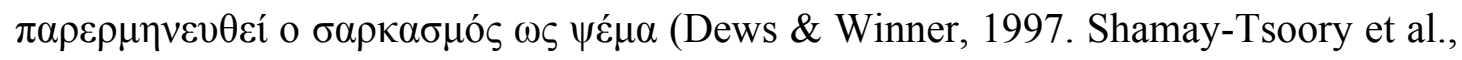
2005. Winner \& Leekman, 1991).

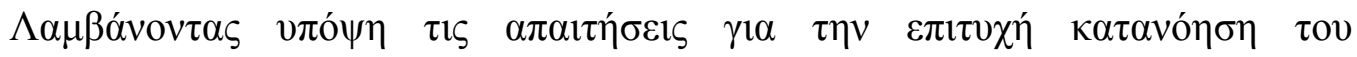

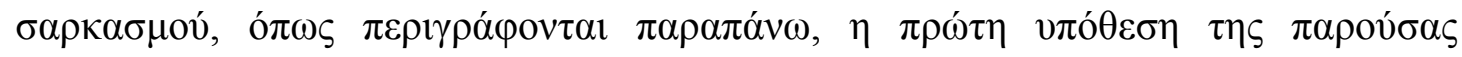

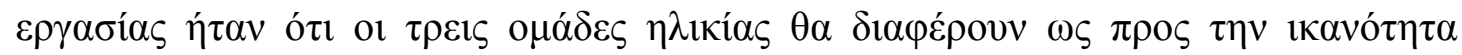

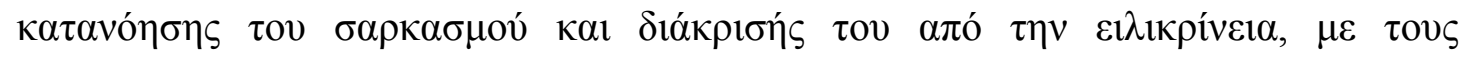

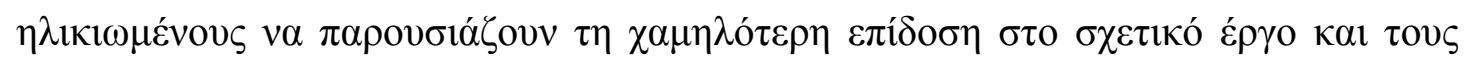

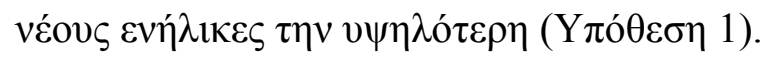

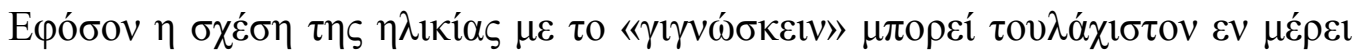

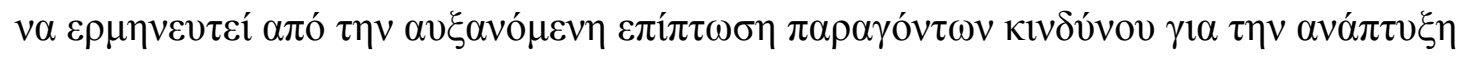

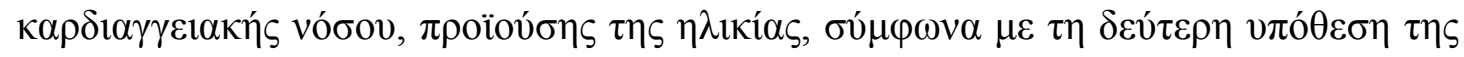
$\pi \alpha \rho o v ́ \sigma \alpha \varsigma \quad \mu \varepsilon \lambda \varepsilon \dot{\tau} \eta \varsigma, \quad \eta \quad \alpha v \tau 0-\alpha v \alpha \varphi \varepsilon \rho o ́ \mu \varepsilon v \eta \quad \delta i \alpha ́ \gamma v \omega \sigma \eta \quad v \pi \varepsilon \rho \chi 0 \lambda \eta \sigma \tau \varepsilon \rho 1 v \alpha \mu i_{\alpha} \alpha \varsigma \quad \theta \alpha$

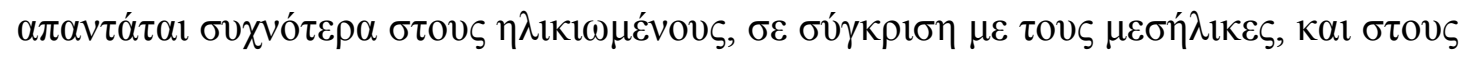

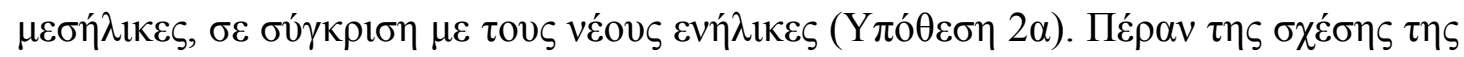

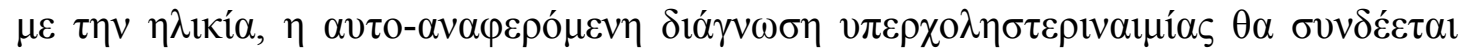

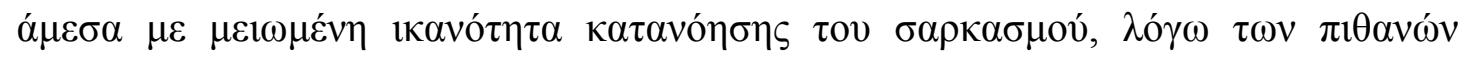

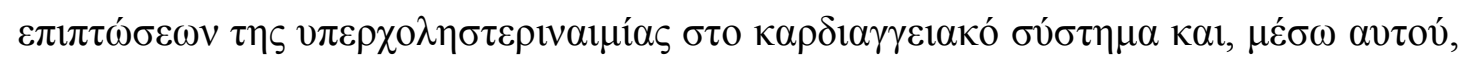

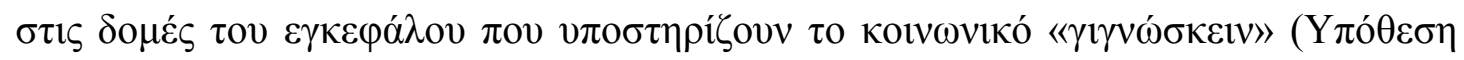
$2 \beta)$. 


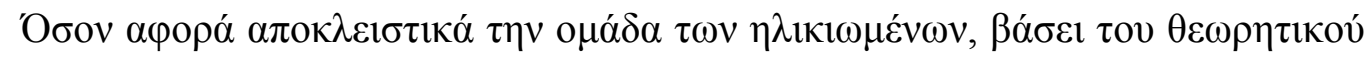

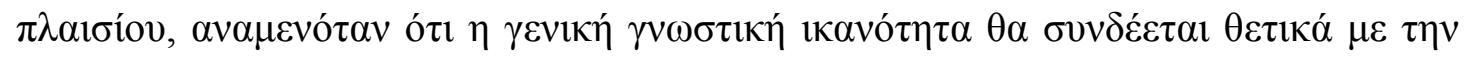

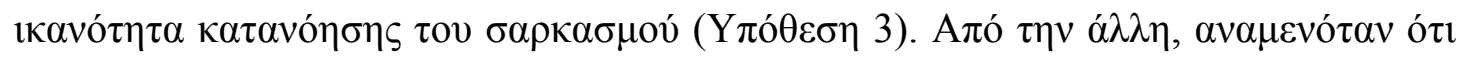

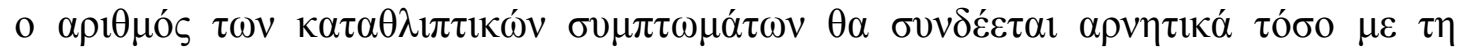

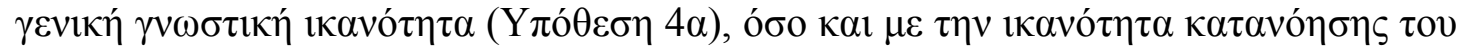
$\sigma \alpha \rho \kappa \alpha \sigma \mu о u ́(Y \pi o ́ \theta \varepsilon \sigma \eta 4 \beta)$. 


\section{$\operatorname{ME\Theta O} \Delta O \Sigma$}

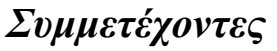

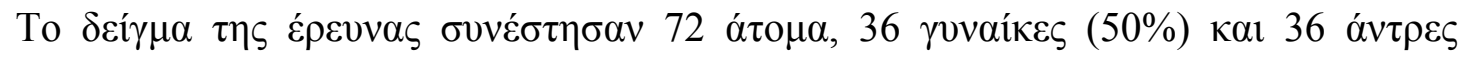

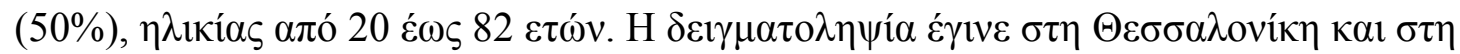

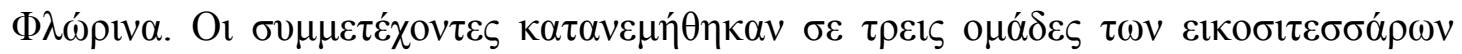

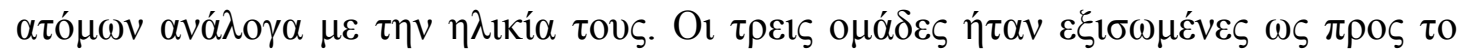

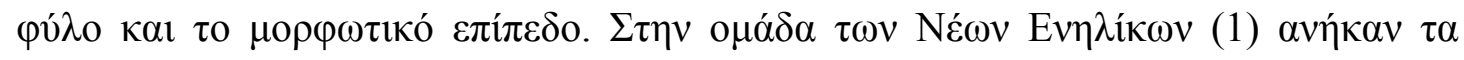

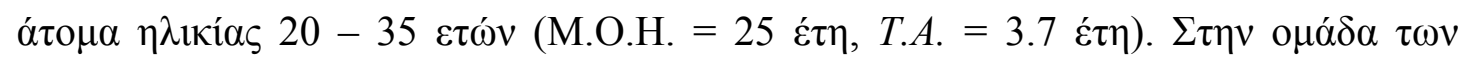

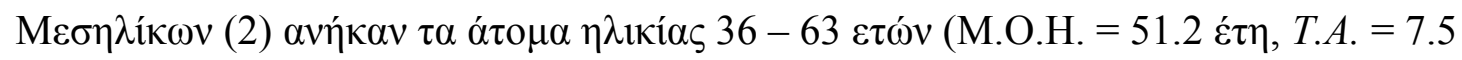

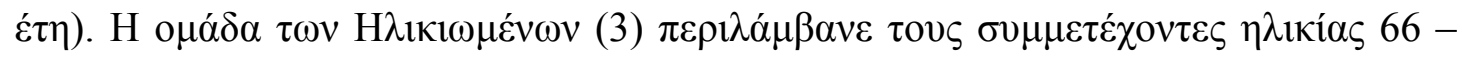

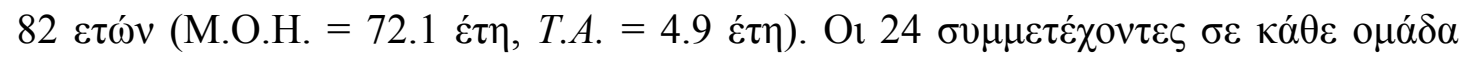

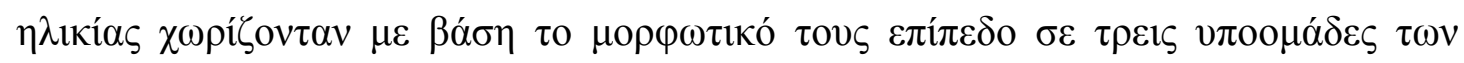

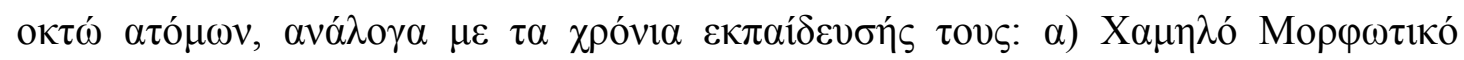

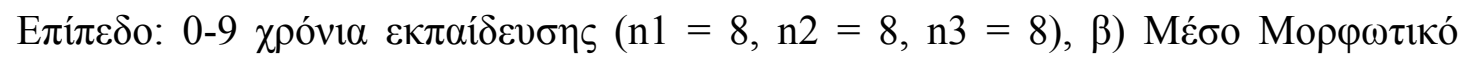

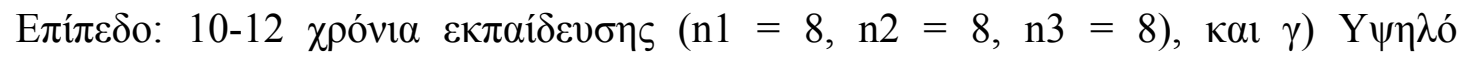

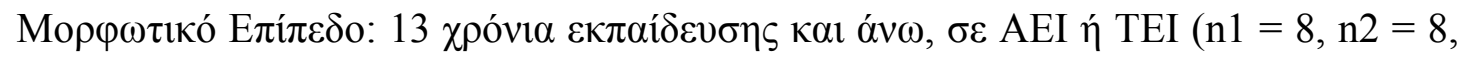

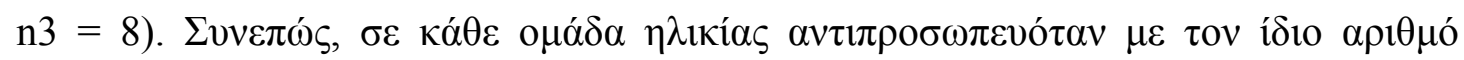

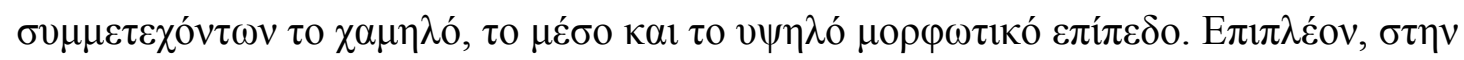

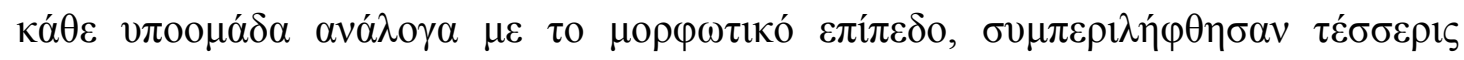

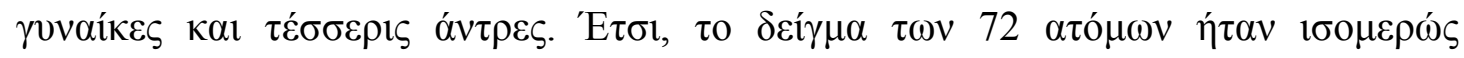

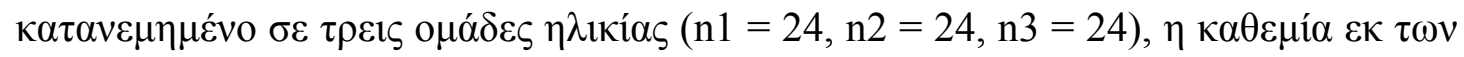

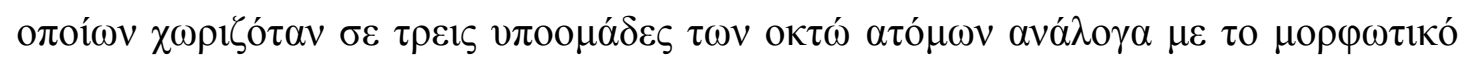

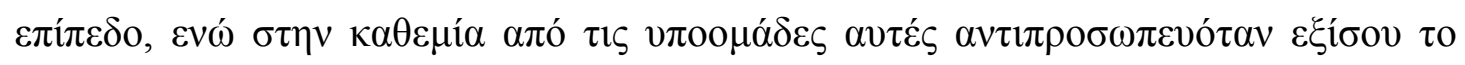

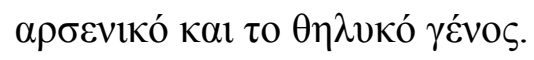




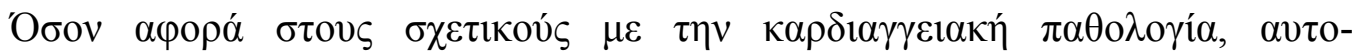

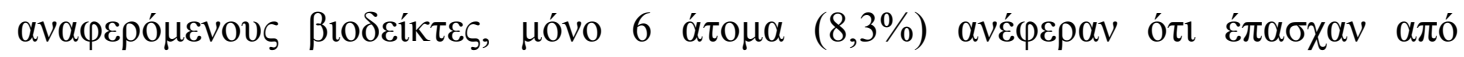

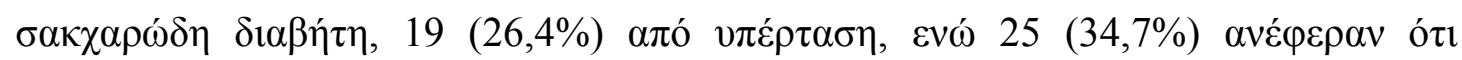

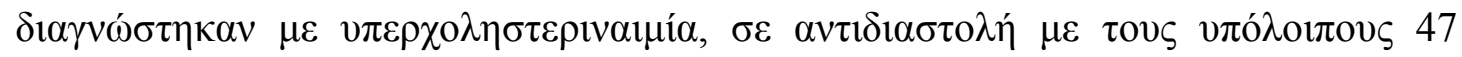
$(65,3 \%)$

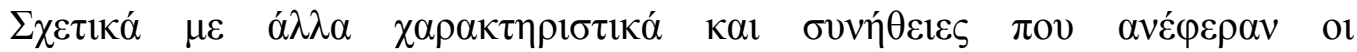

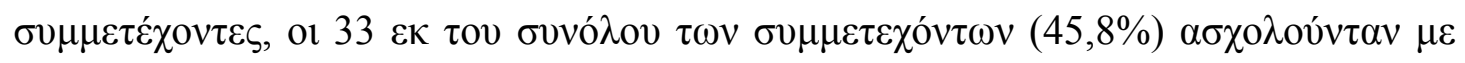

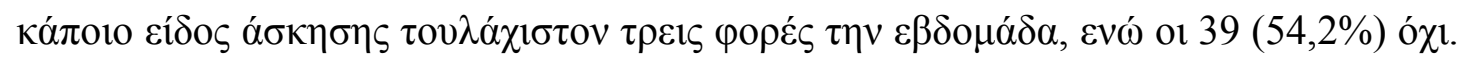
Гı

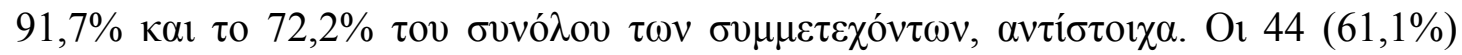

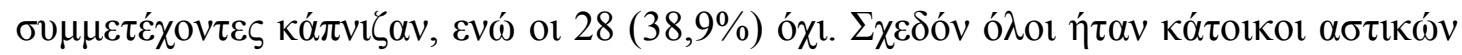
$\pi \varepsilon \rho 10 \chi \omega ́ v(90,3 \%)$.

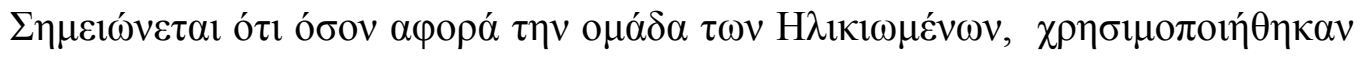

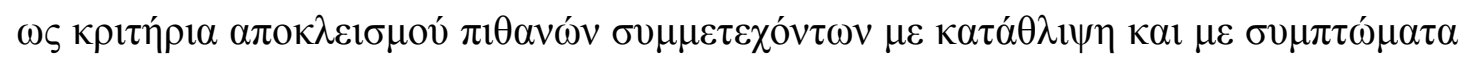

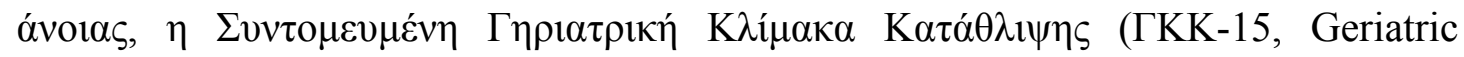

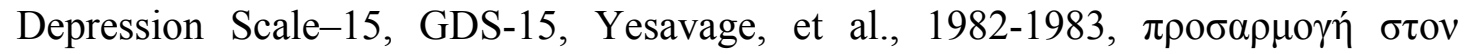

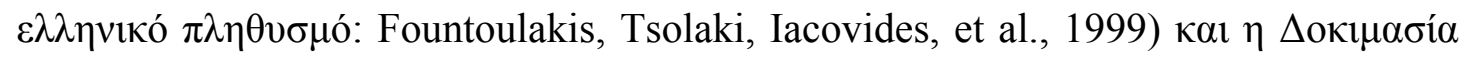

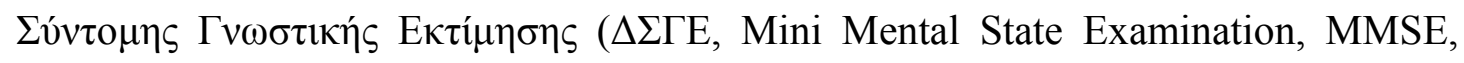

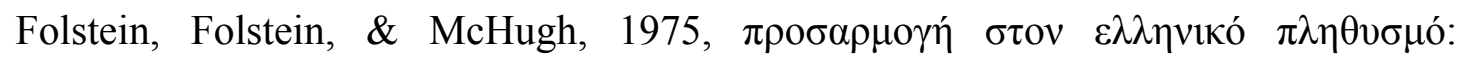

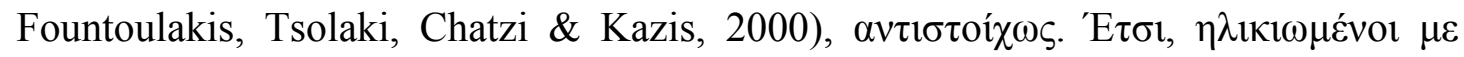

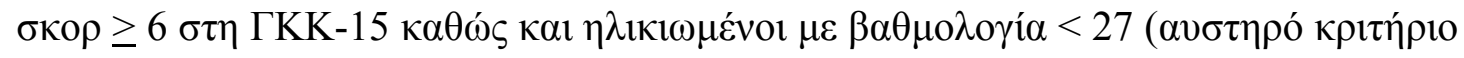

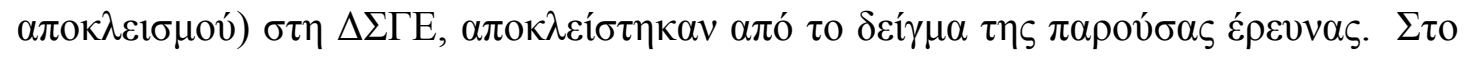

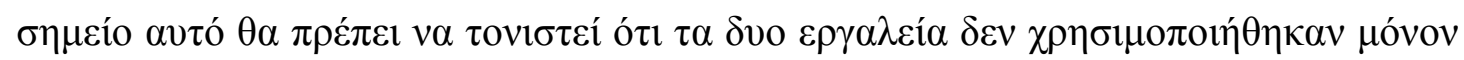

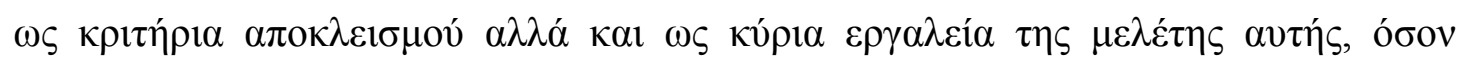

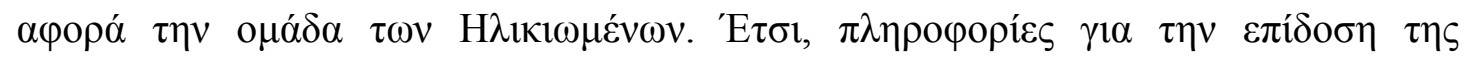




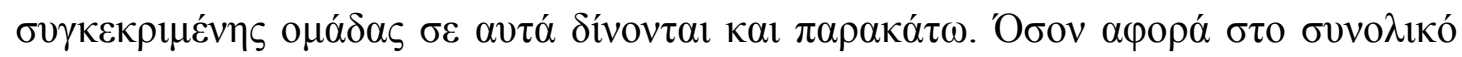

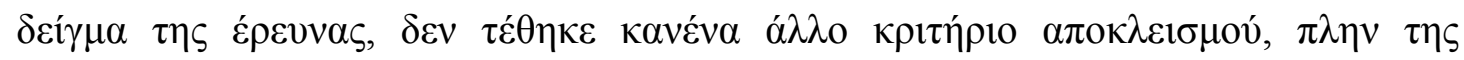

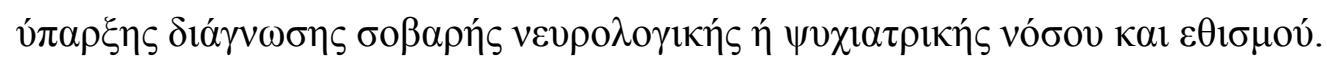

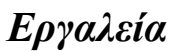

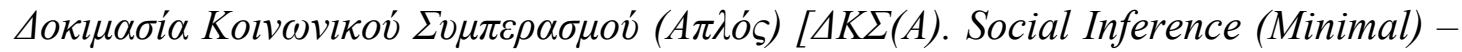
SI(M): Part 2 of The Awareness of Social Inference Test - TASIT, McDonald, Flanagan, Rollins, \& Kinch, 2003]

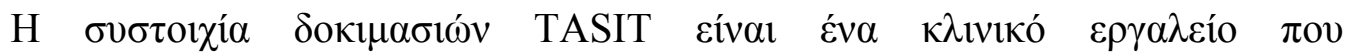

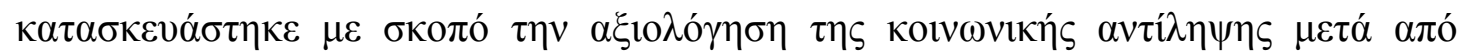

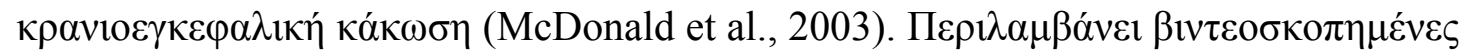

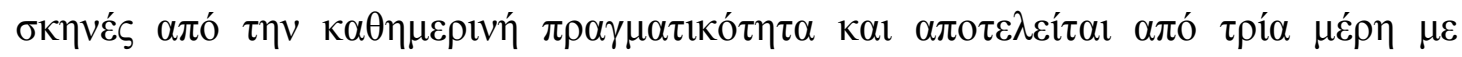

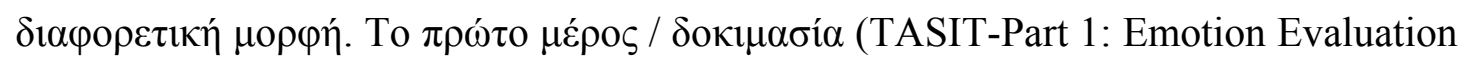

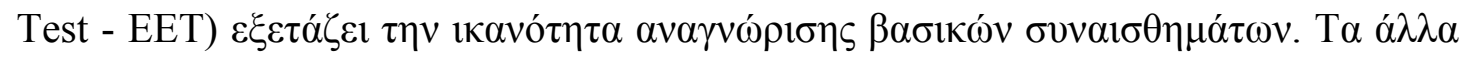

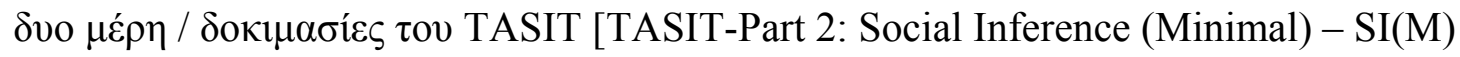

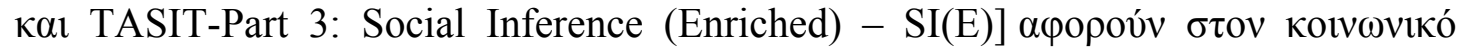

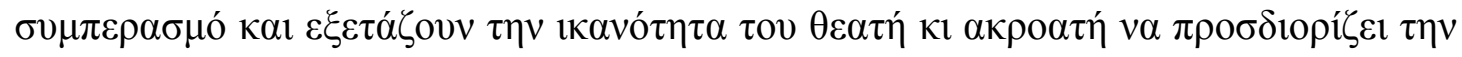

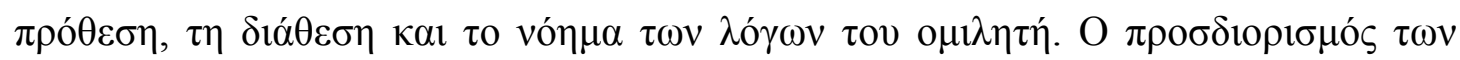

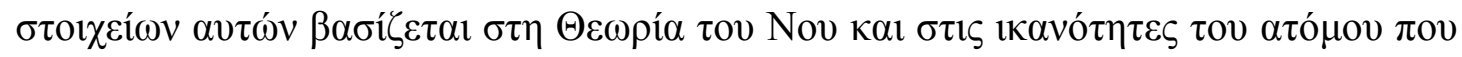

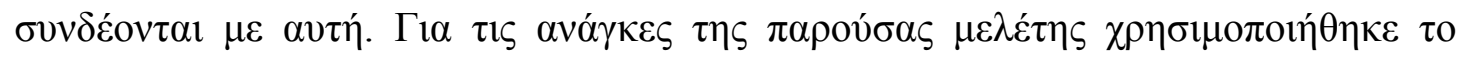

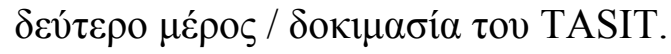

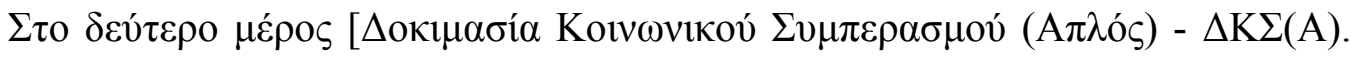

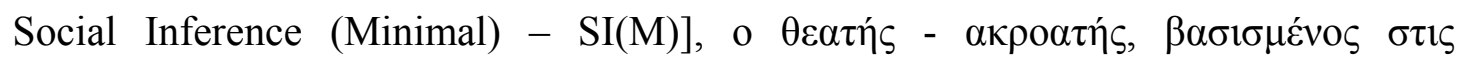

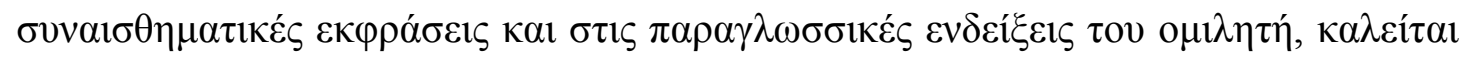

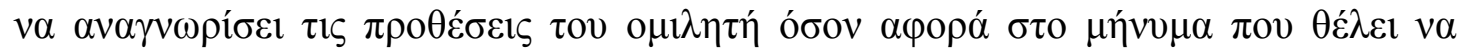




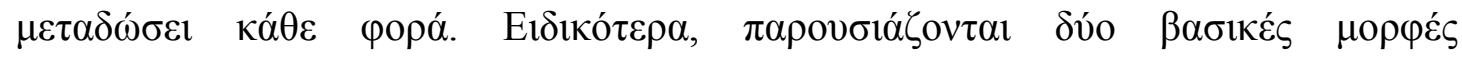

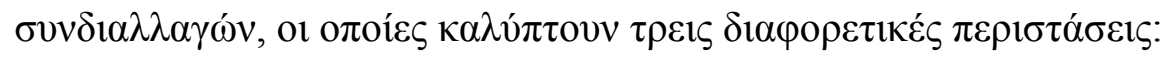

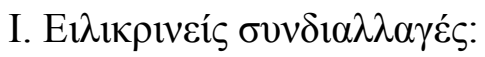

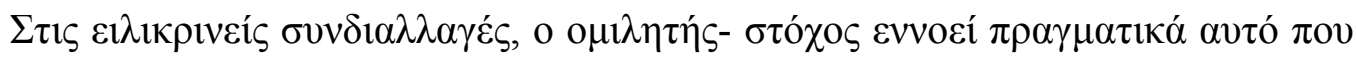

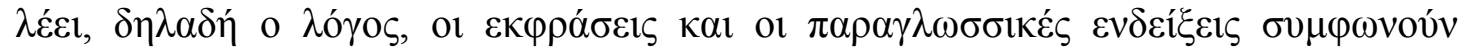
$\mu \varepsilon \tau \alpha \xi \dot{v} \tau o v \varsigma$.

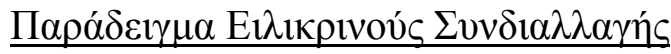

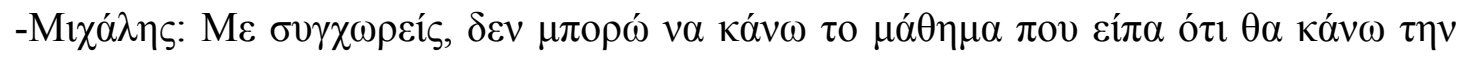

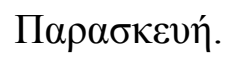

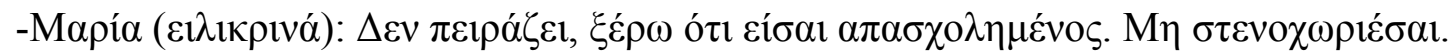

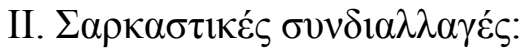

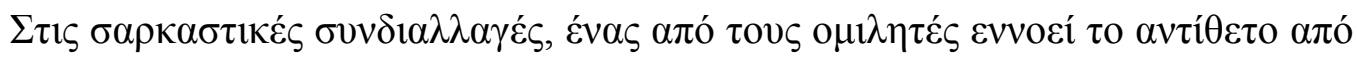

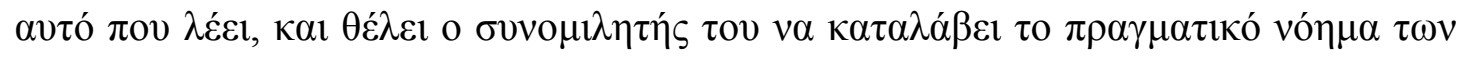

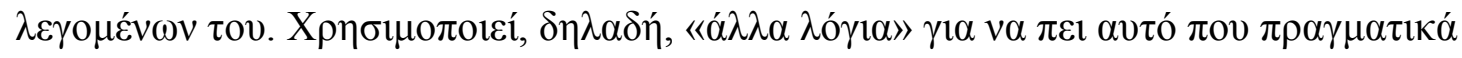

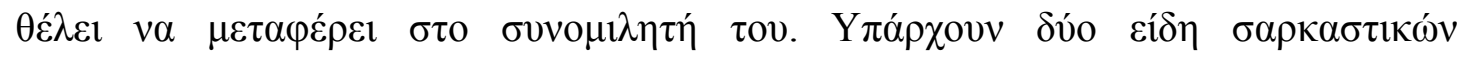

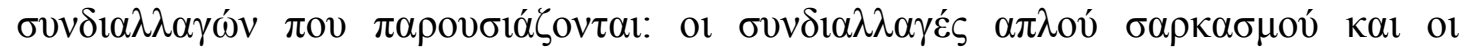
$\sigma v v \delta 1 \alpha \lambda \lambda \alpha \gamma \varepsilon \dot{\zeta} \varsigma \alpha \rho \alpha ́ \delta$ o

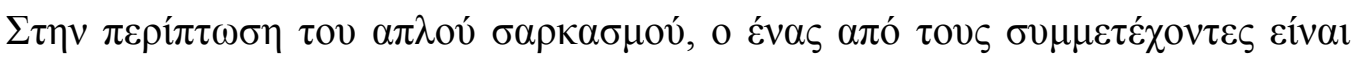

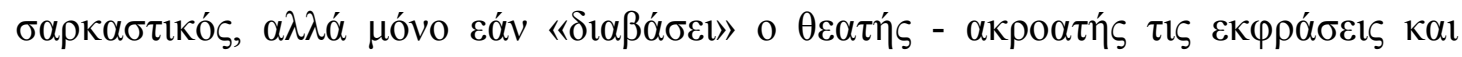

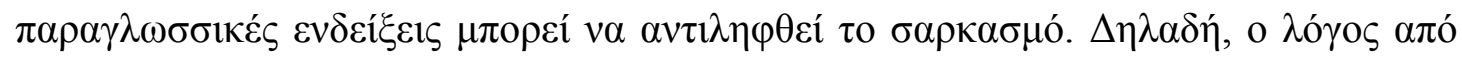

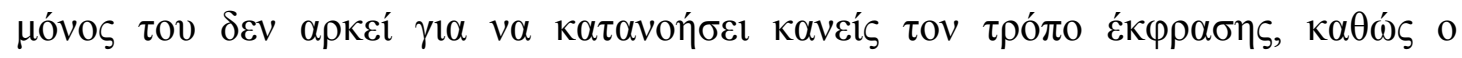

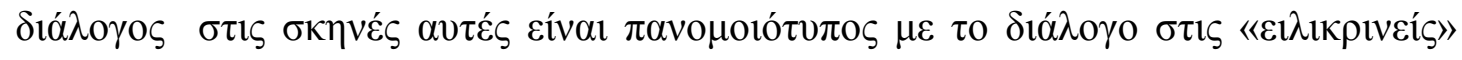

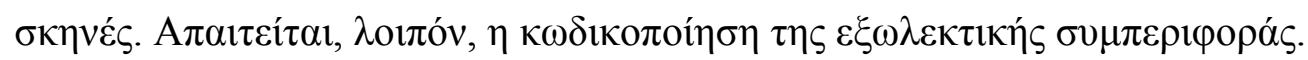




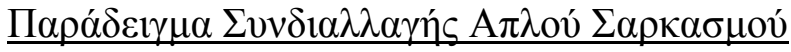

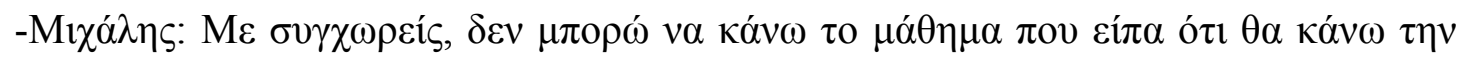

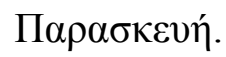

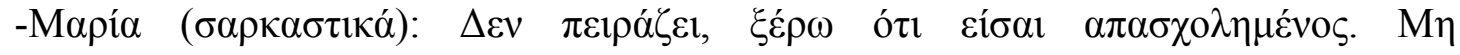
$\sigma \tau \varepsilon v 0 \chi \omega \rho t \varepsilon ́ \sigma \alpha 1$.

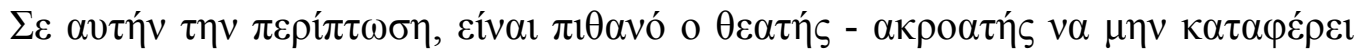

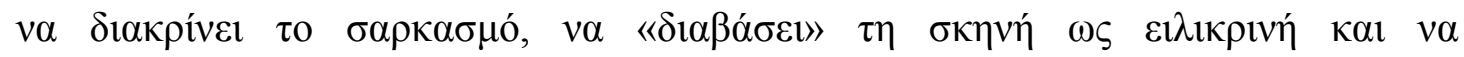

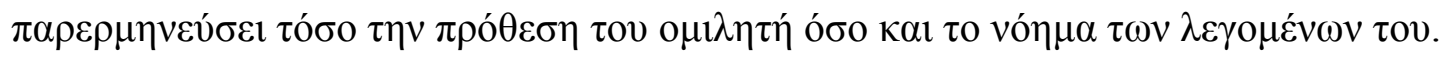

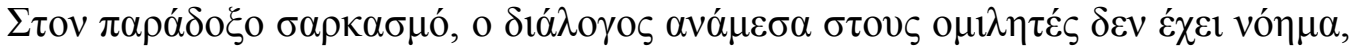

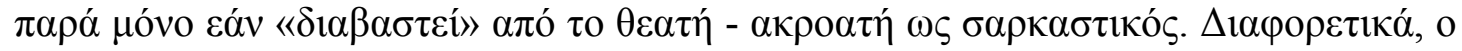

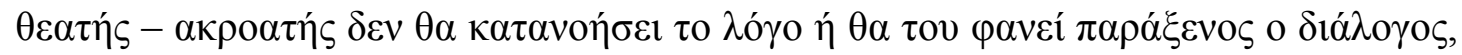

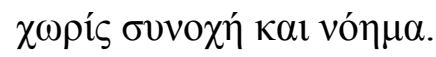

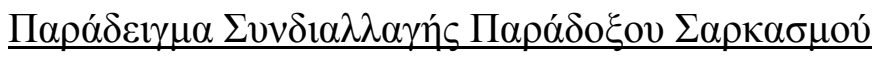

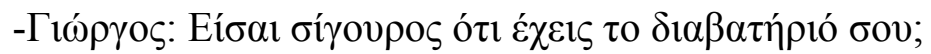

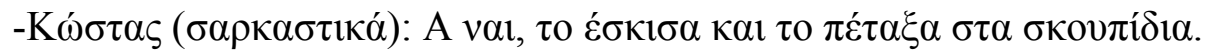

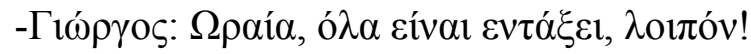

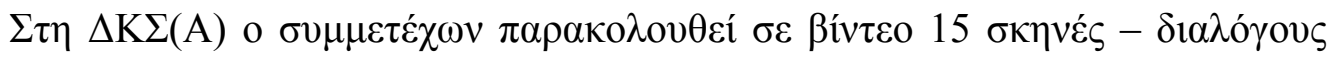

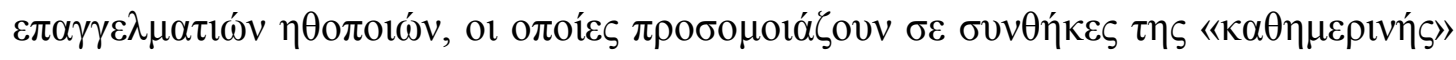

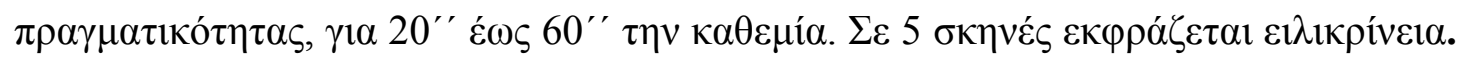

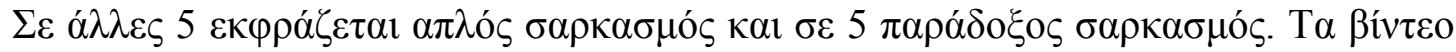

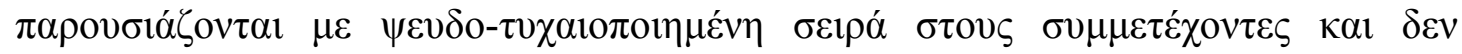

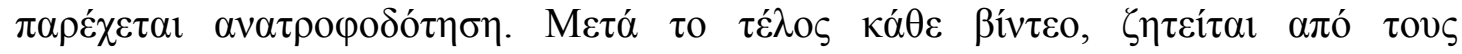

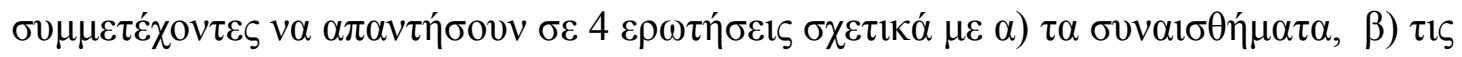

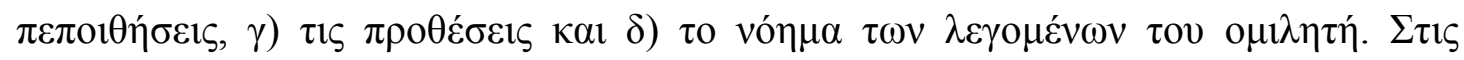

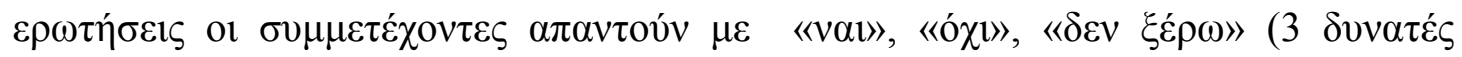




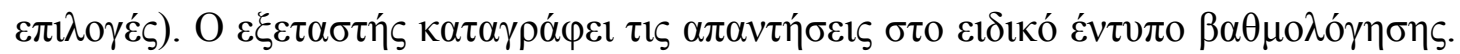

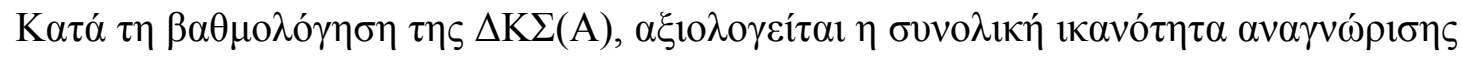

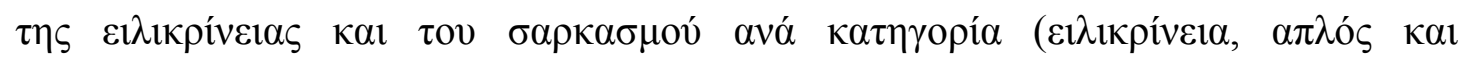

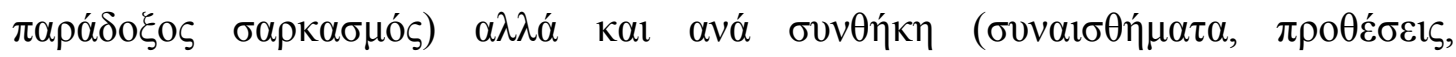

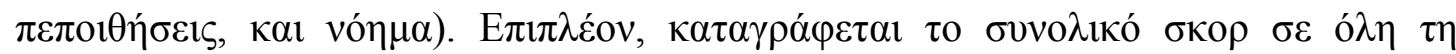

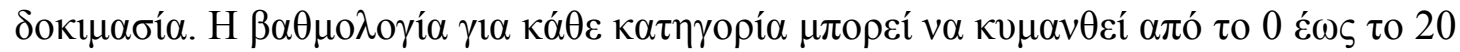

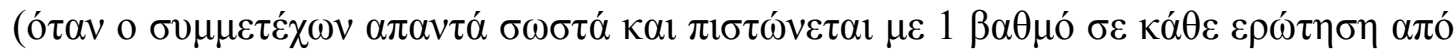

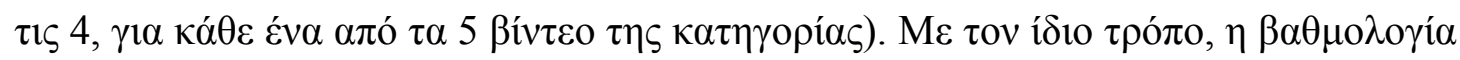

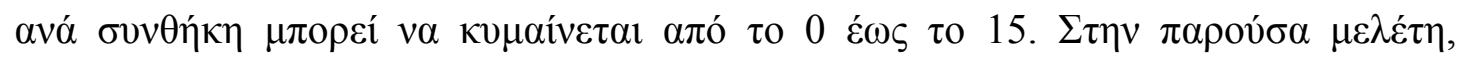

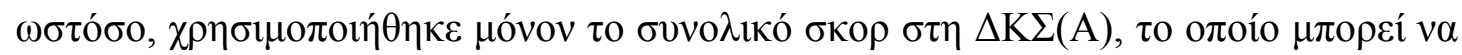

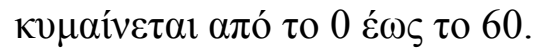

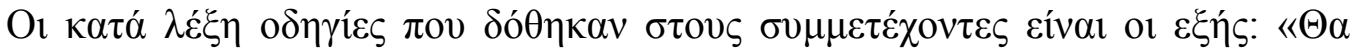

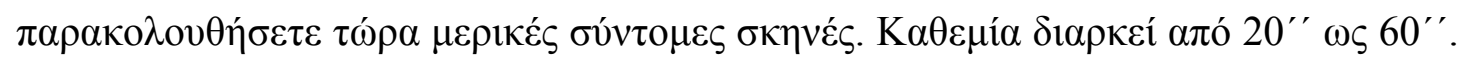

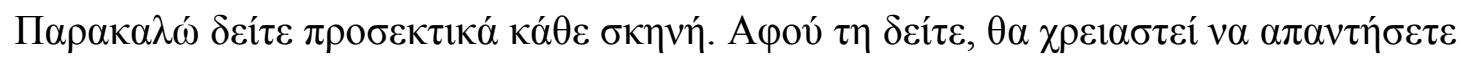

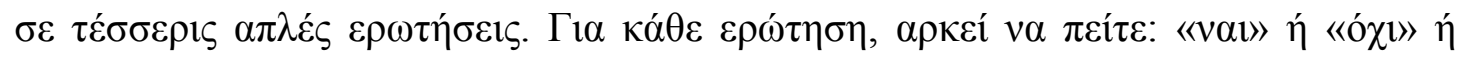

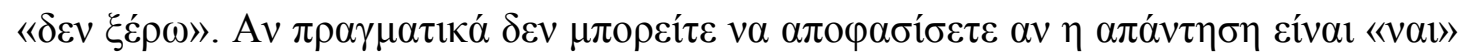

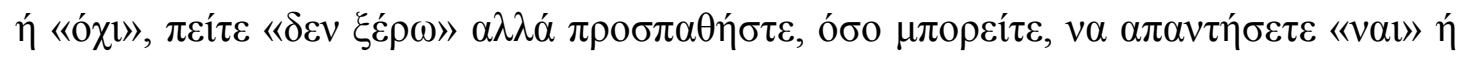

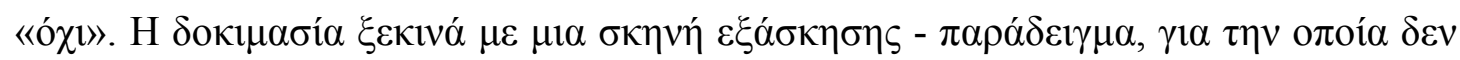

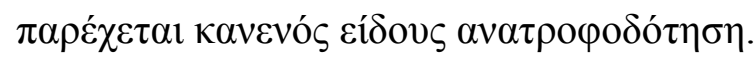

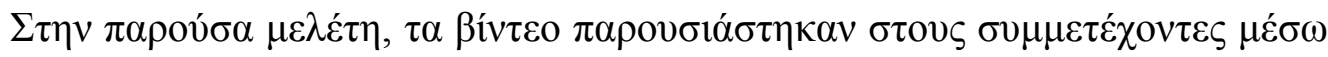

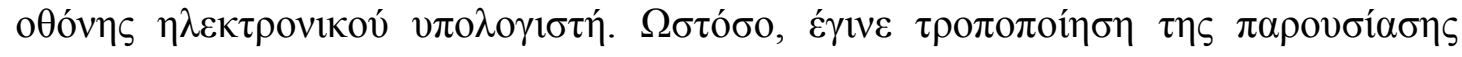

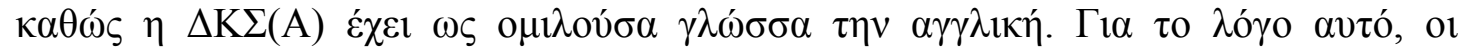

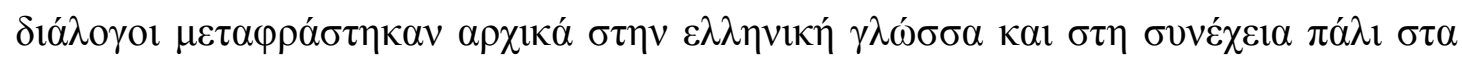

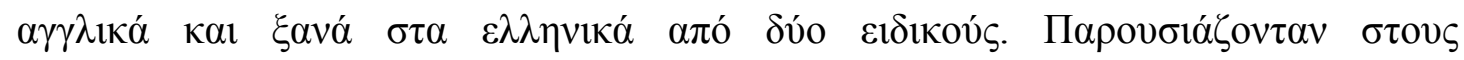

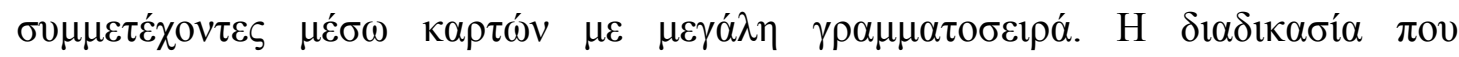




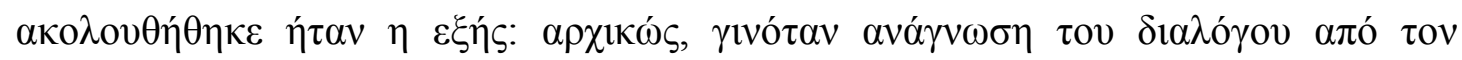

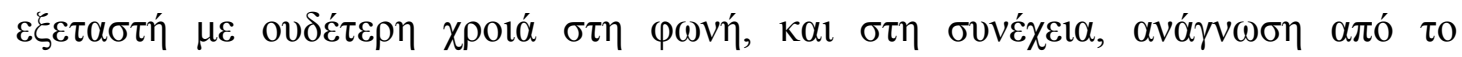

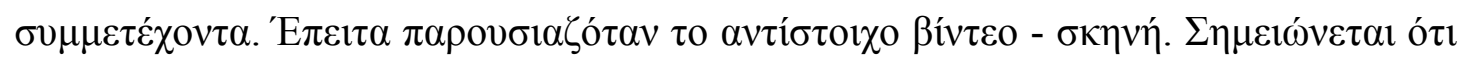

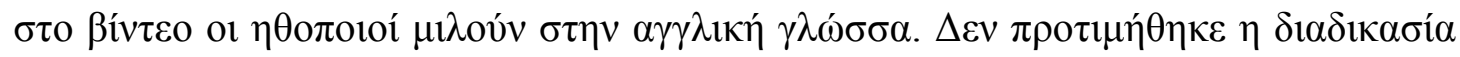

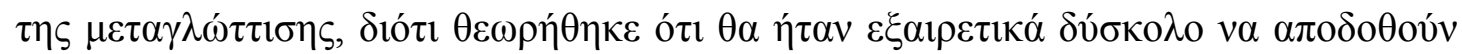

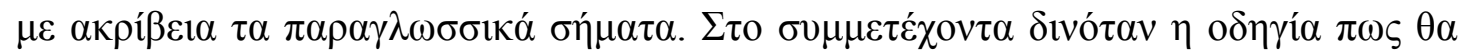

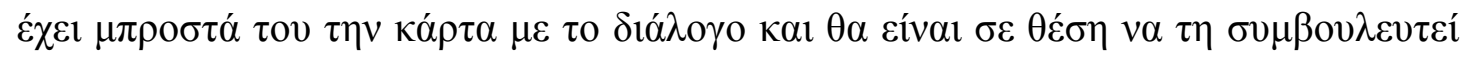

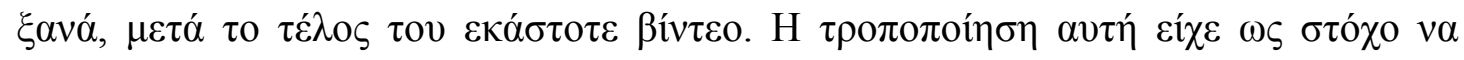

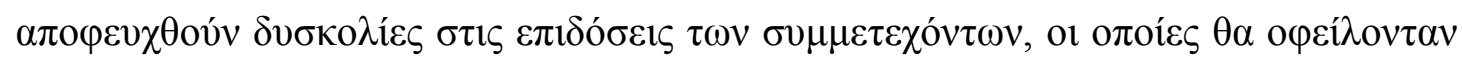

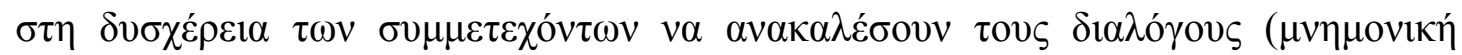
$\lambda \varepsilon i \tau o v \rho \gamma i \alpha)$.

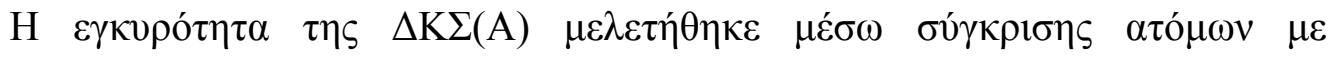

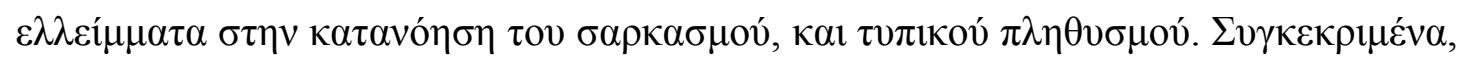

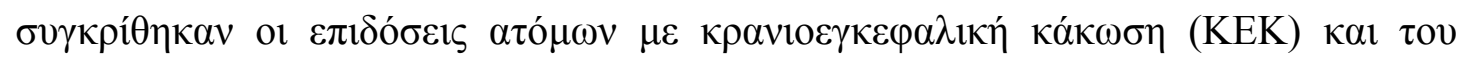

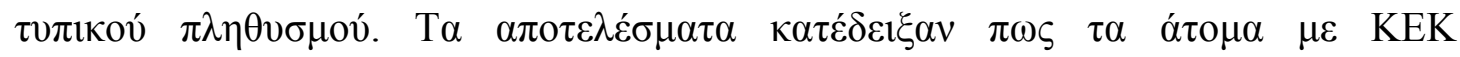

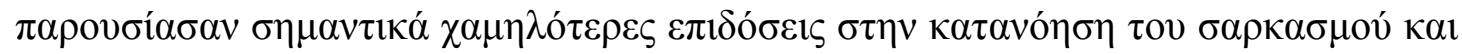

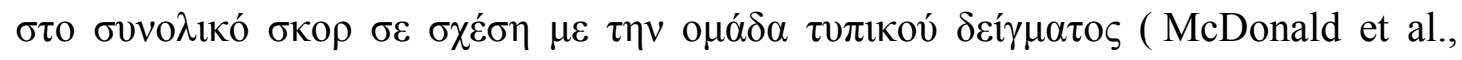

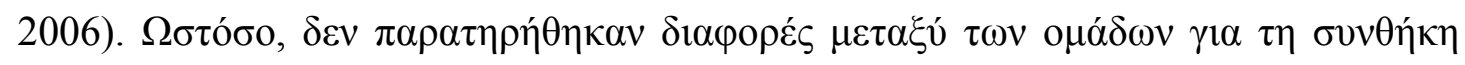

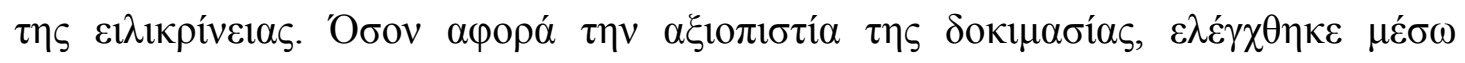

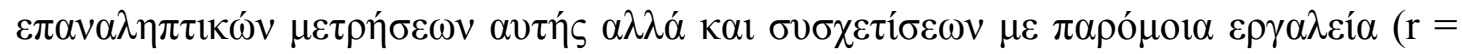

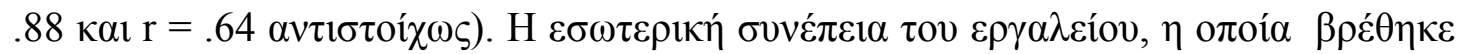

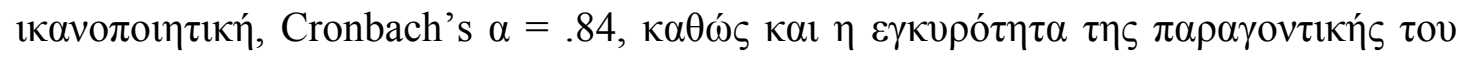

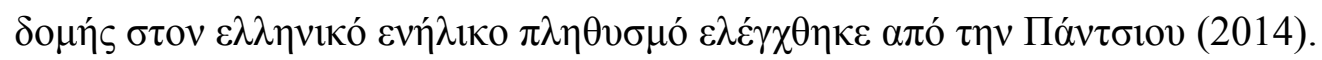




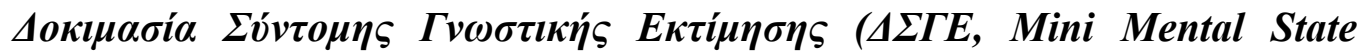

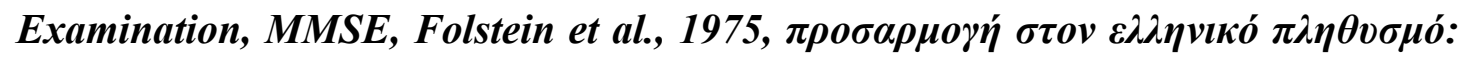
Fountoulakis, et al., 2000)

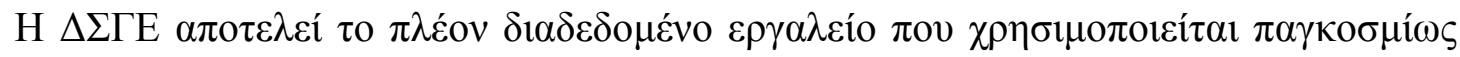

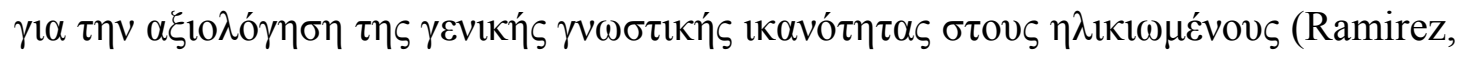
Teresi, Holmes, Gurland, \& Lantigua, 2006. Shigemori, Ohgi, Okuyama, Shimura, \&

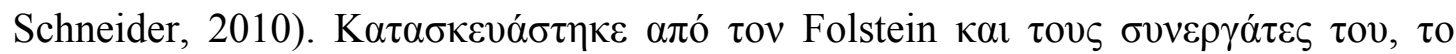

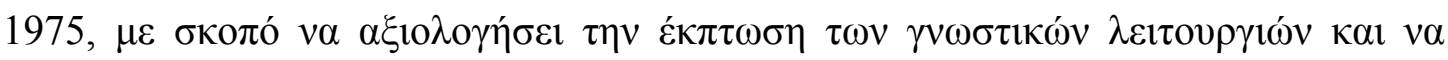

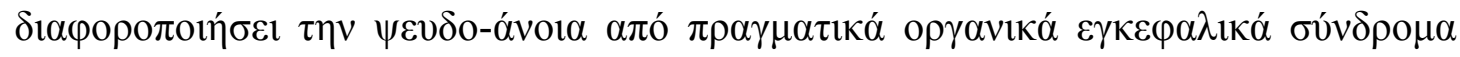

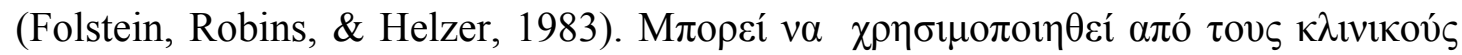

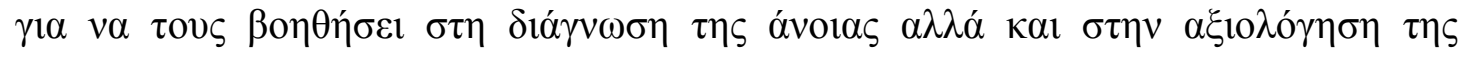

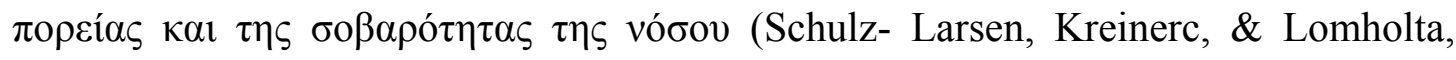

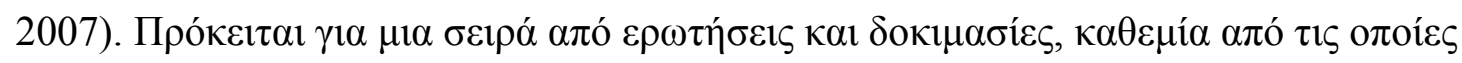

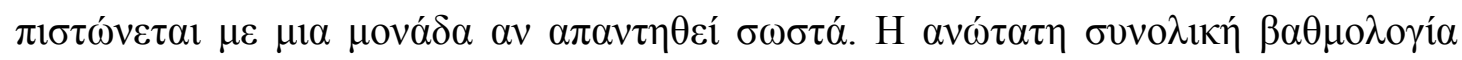

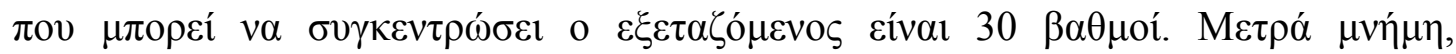

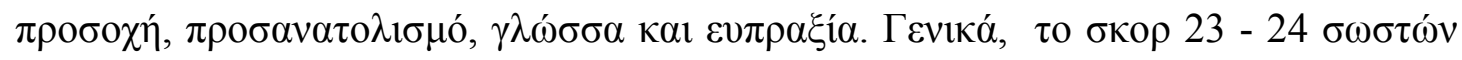

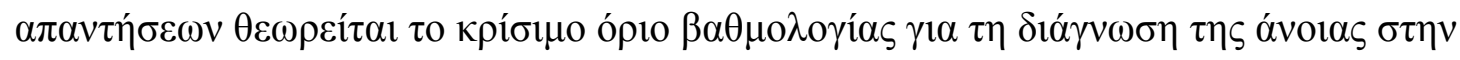

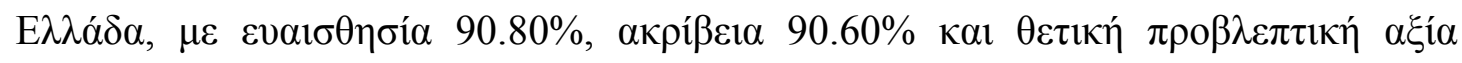

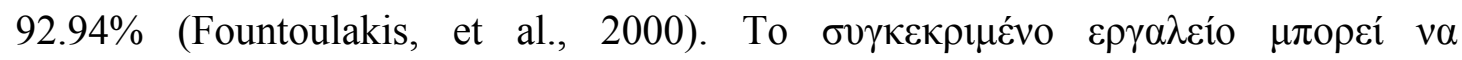

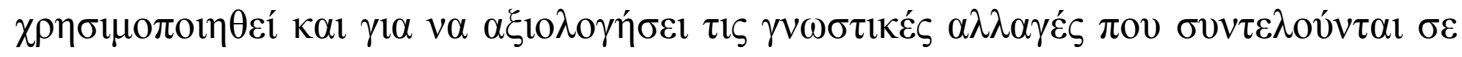

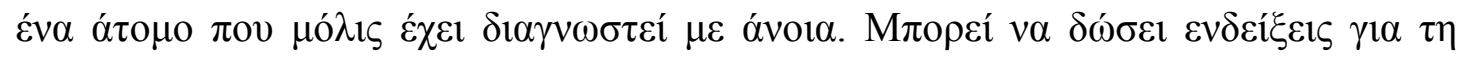

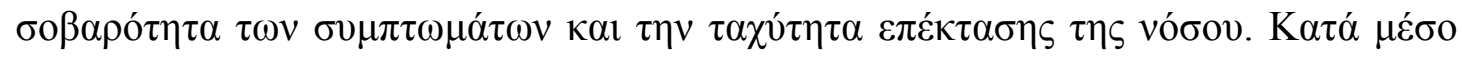

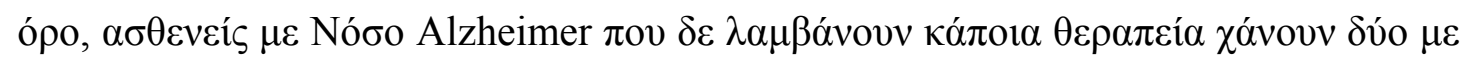

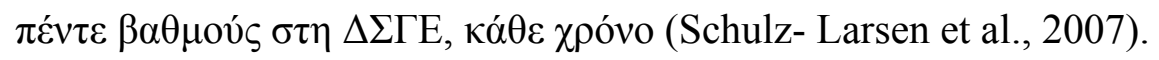




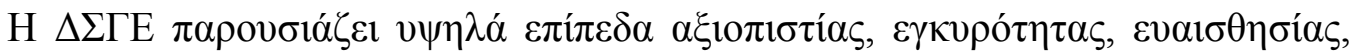

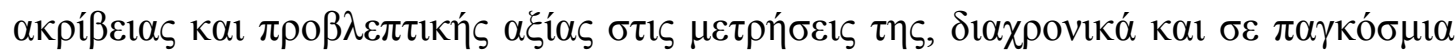
$\varepsilon \mu \beta \varepsilon ́ \lambda \varepsilon ı \alpha$ (Mitchell, 2009. Morales, Flowers, Gutierrez, Kleinman, \& Teresi, 2006. O’ Connor et al., 1989. Ramirez et al., 2006).

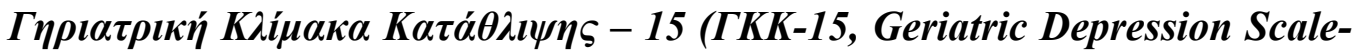

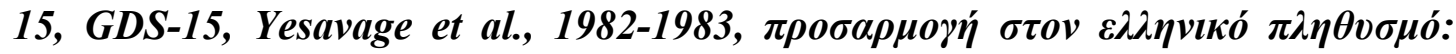
Fountoulakis et al., 1999)

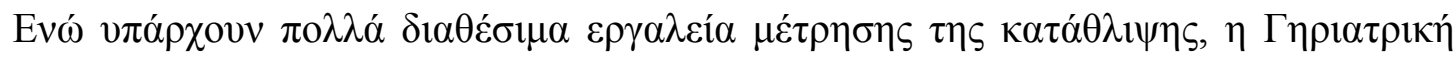

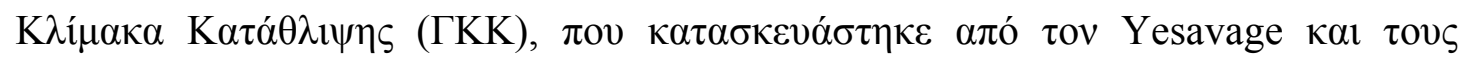

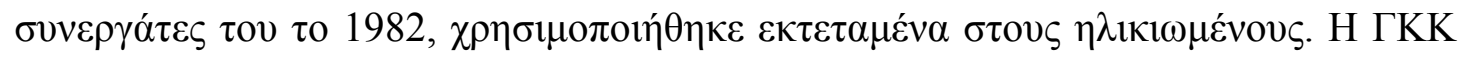

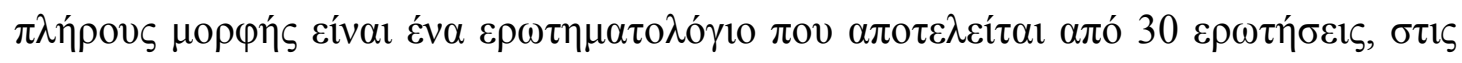

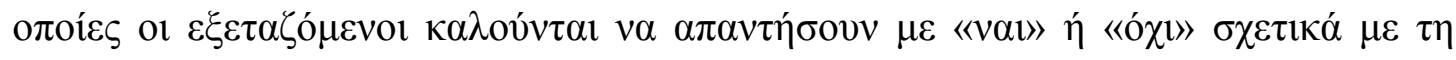

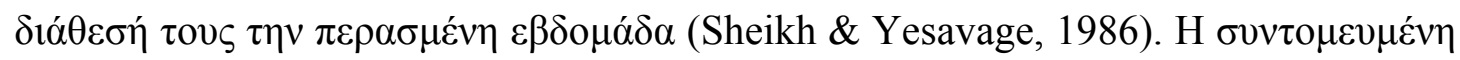

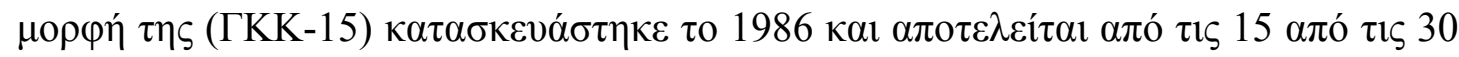

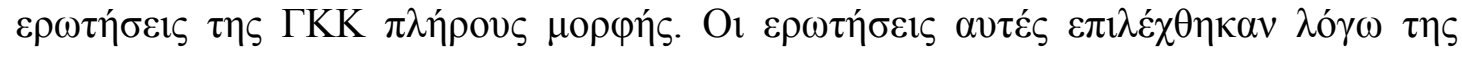

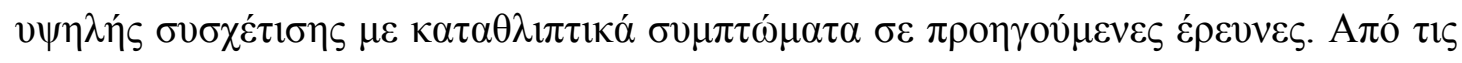

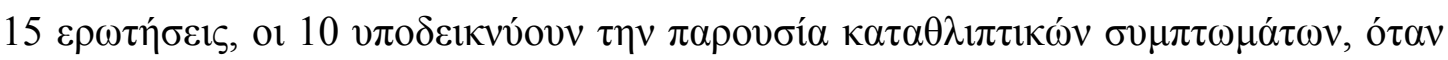

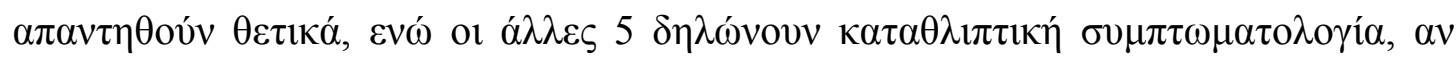

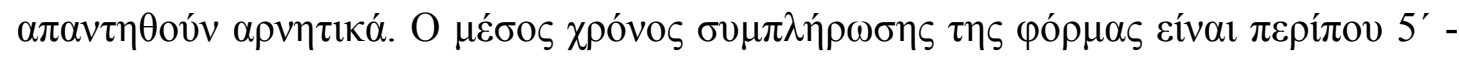

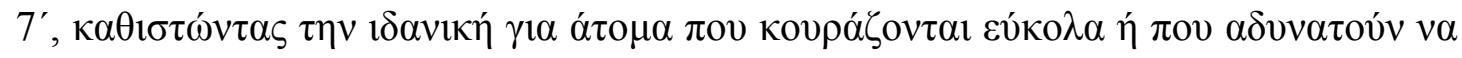

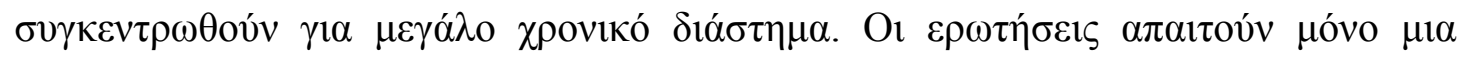

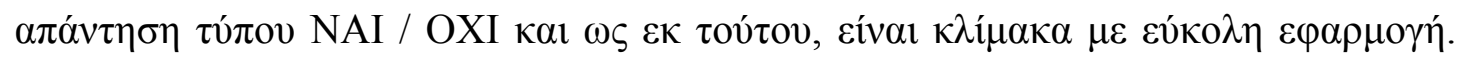

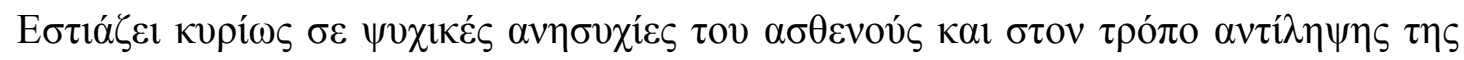

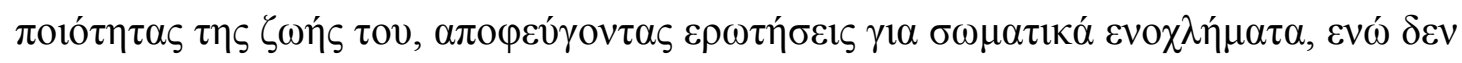




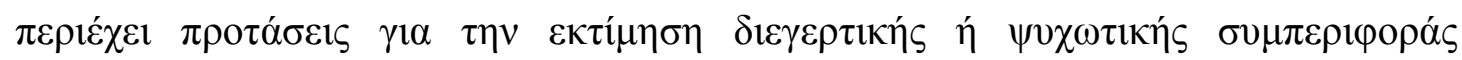

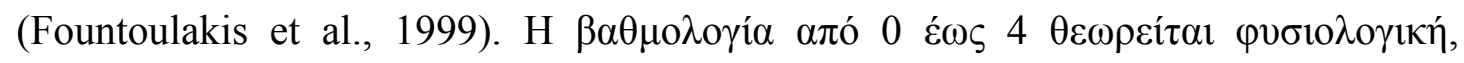

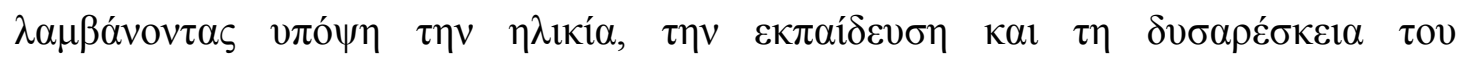

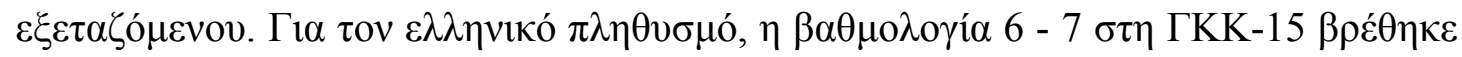

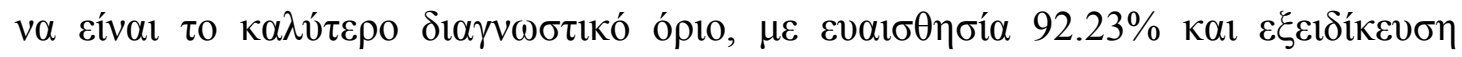

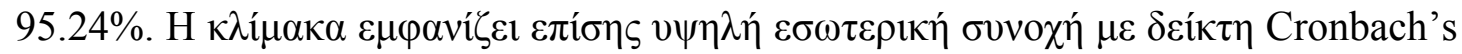

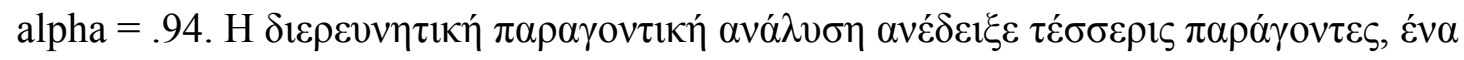

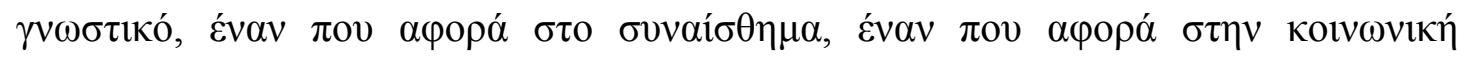

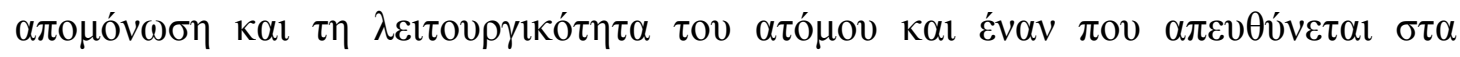

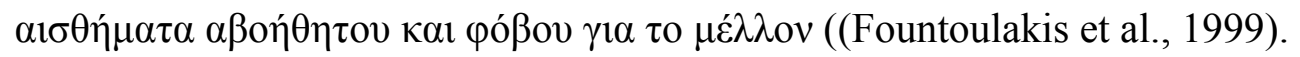

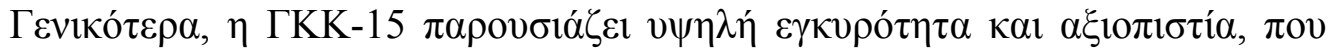

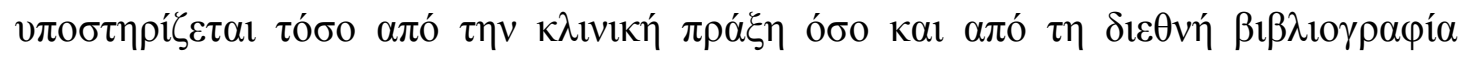

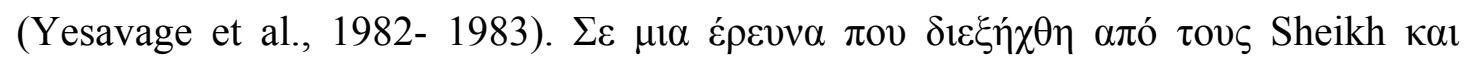

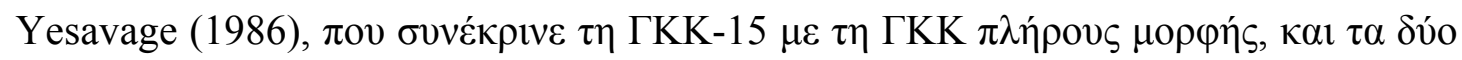

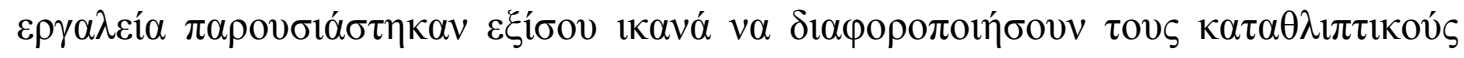

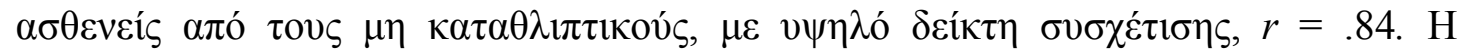

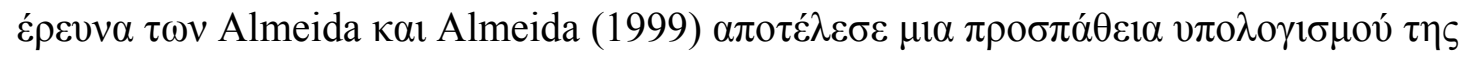

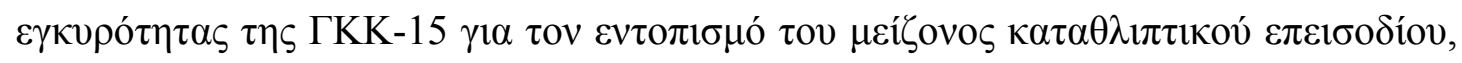

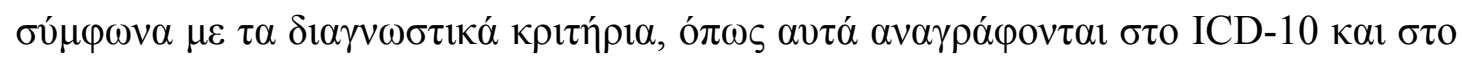

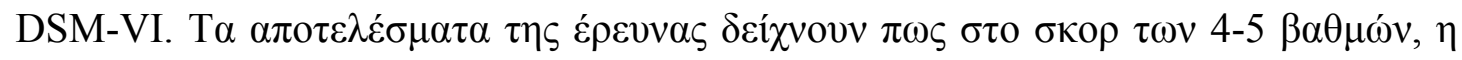

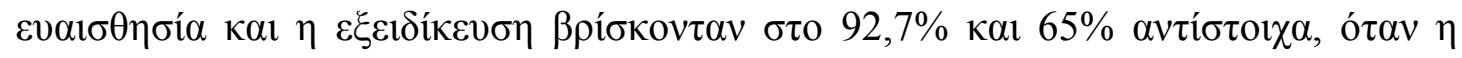

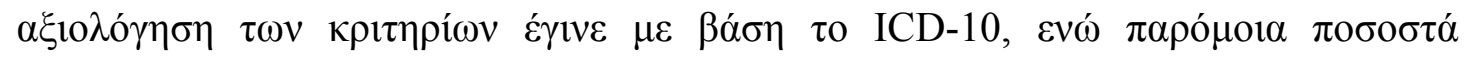

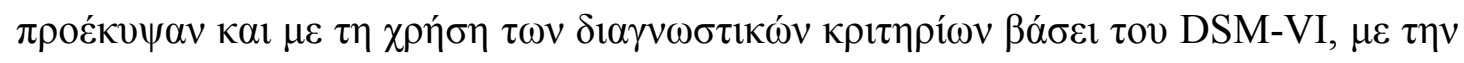

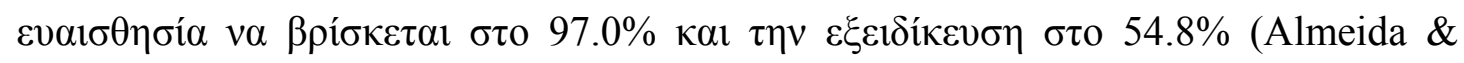

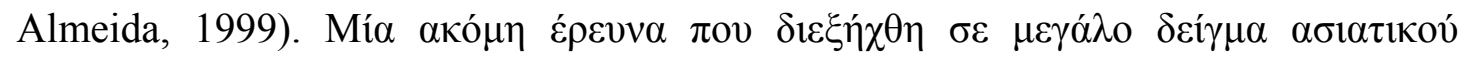




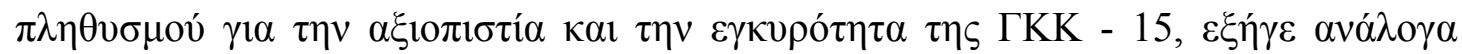

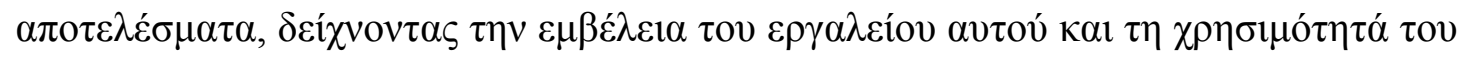

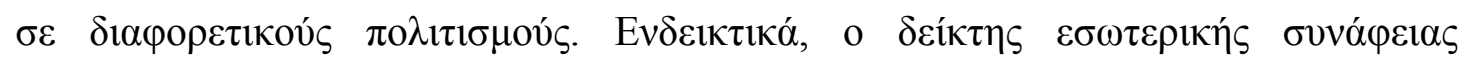

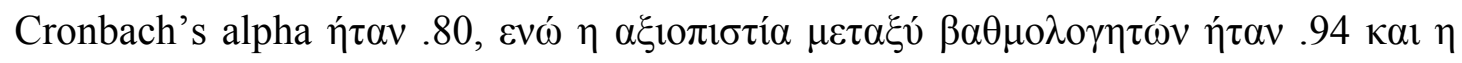
$\alpha \xi ı \pi ı \tau \tau i ́ \alpha \varepsilon \pi \alpha v \alpha \lambda \eta \pi \tau ı \kappa \omega ́ v ~ \mu \varepsilon \tau \rho \eta ́ \sigma \varepsilon \omega v ~ .83$ (Nyunt, Fones, \& Niti, 2009).

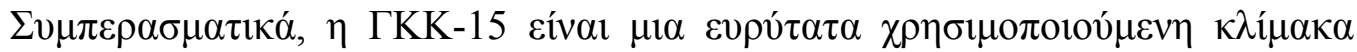

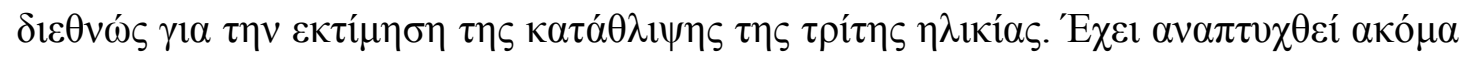

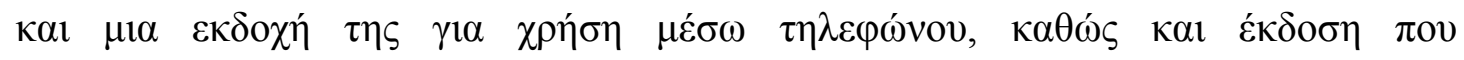

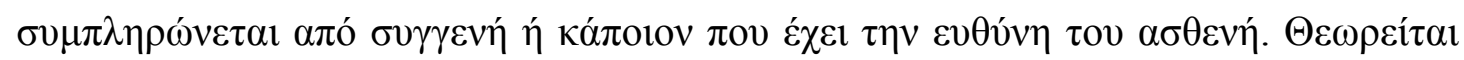

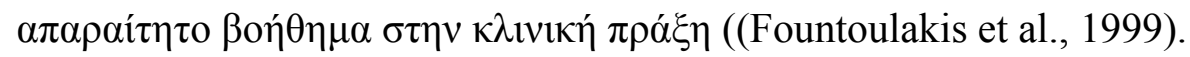

\section{$\Delta \iota \alpha \delta$ ıк $\alpha i ́ \alpha$}

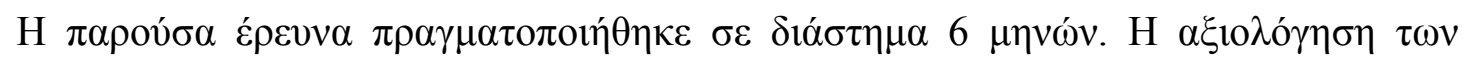

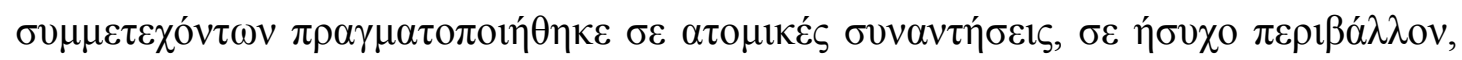

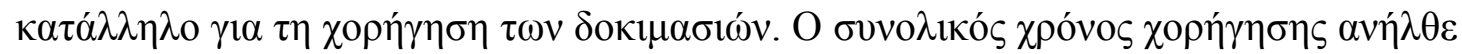

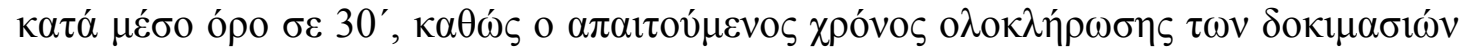

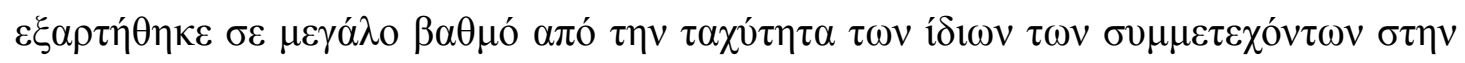

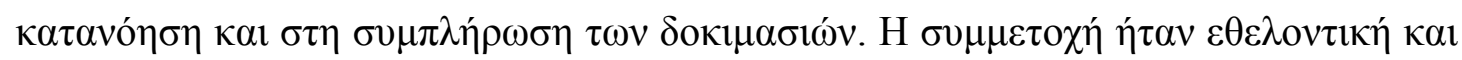

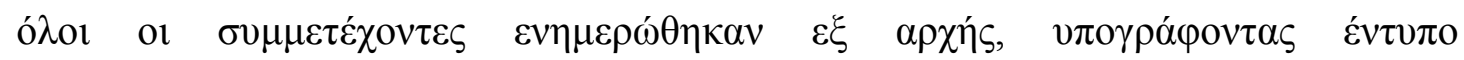

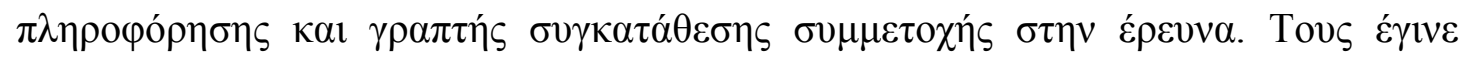

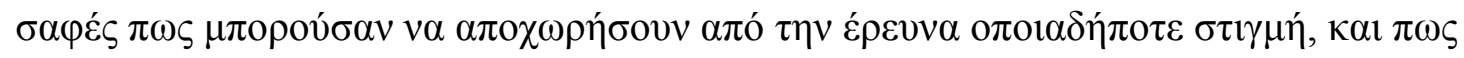

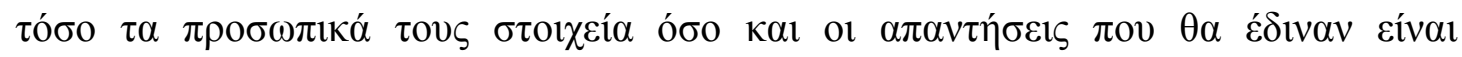

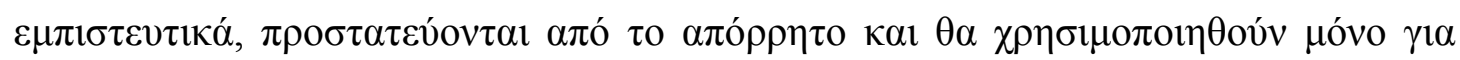

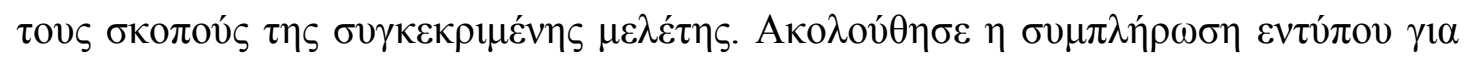
$\alpha \tau$ 


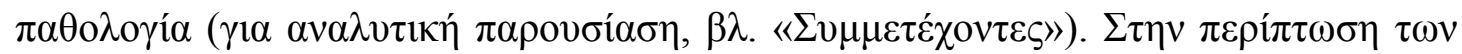

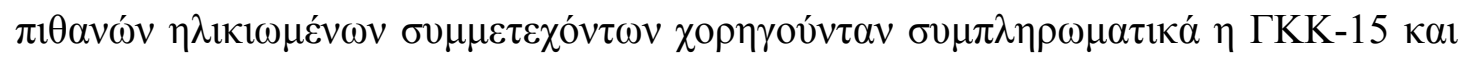

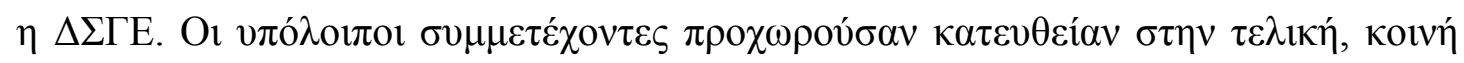

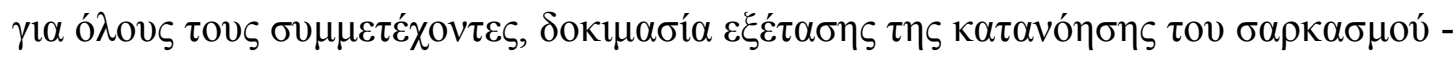
$\Delta \mathrm{K} \Sigma(\mathrm{A})$. 


\section{AПOTE $\Lambda$ EMATA}

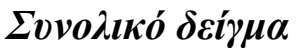

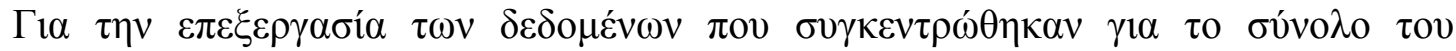

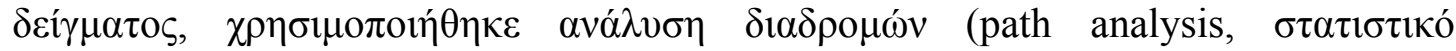

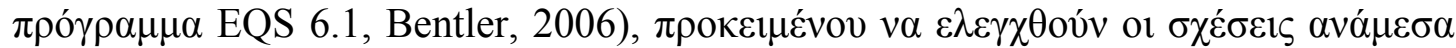

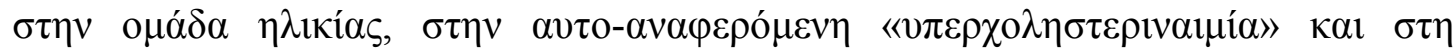

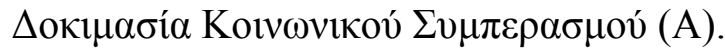

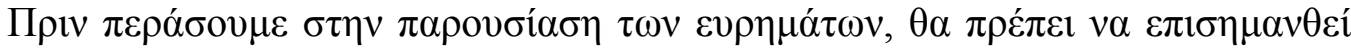

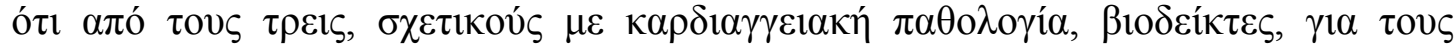

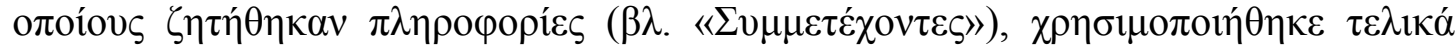

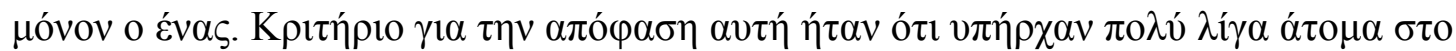

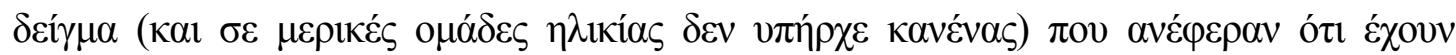

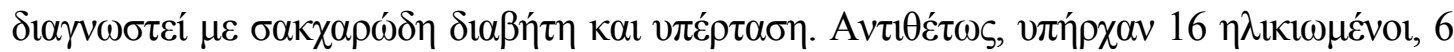

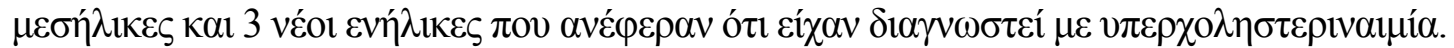

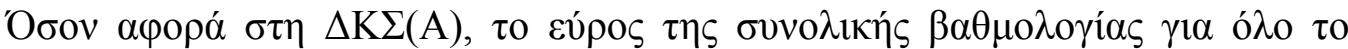

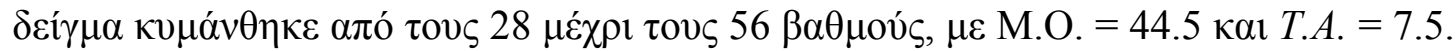

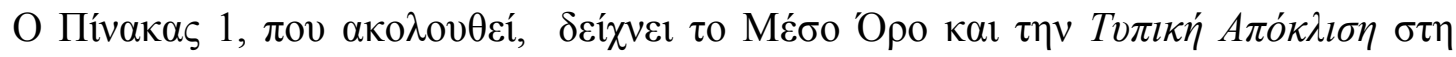

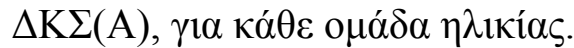




\section{Пívakas 1}

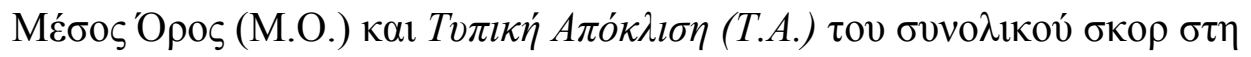

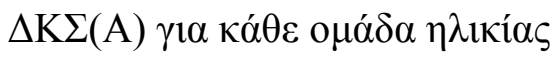

\begin{tabular}{|c|c|c|c|c|c|}
\hline & $N$ & 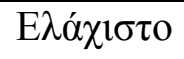 & 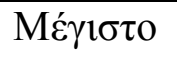 & M.O. & T.A. \\
\hline NÉoı Evท́ & 24 & 37 & 56 & 49.6 & 4.3 \\
\hline Мєбฑ́ & 24 & 31 & 56 & 46.5 & 5.7 \\
\hline 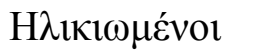 & 24 & 28 & 46 & 37.2 & 5.9 \\
\hline
\end{tabular}

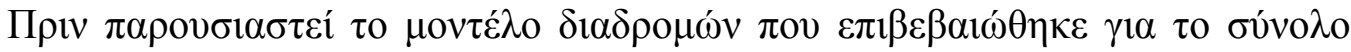

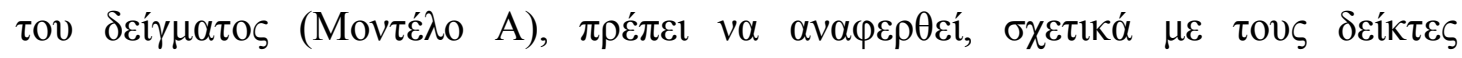

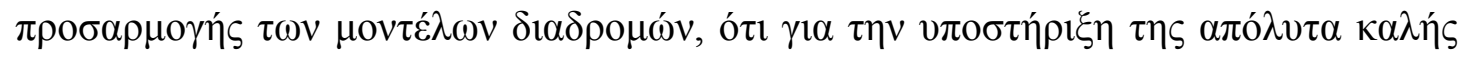

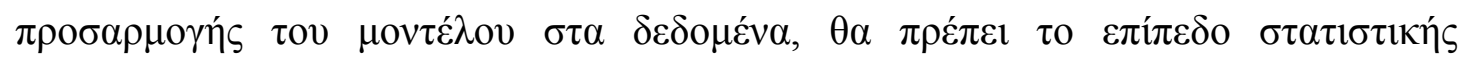

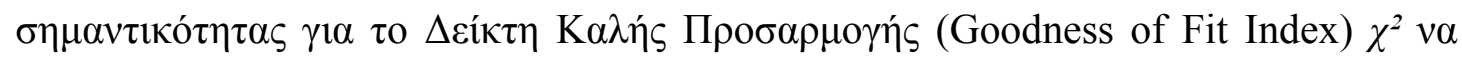

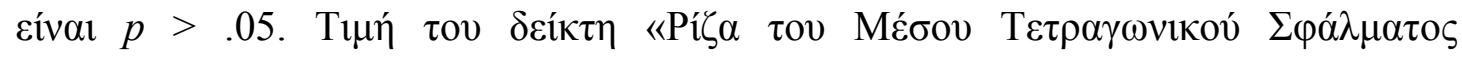

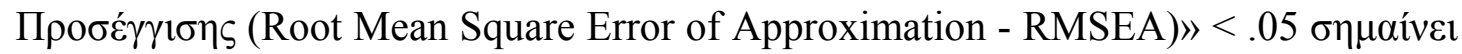

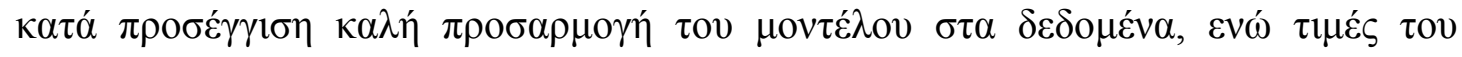

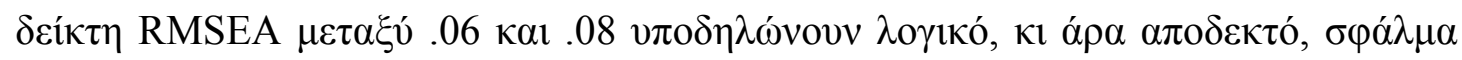

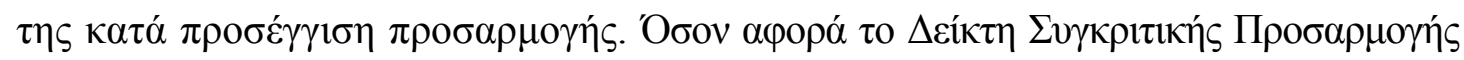

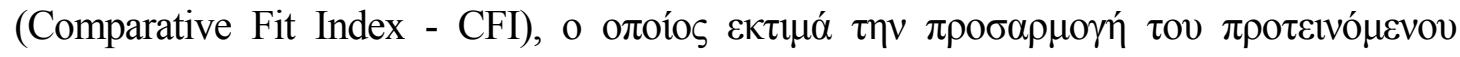

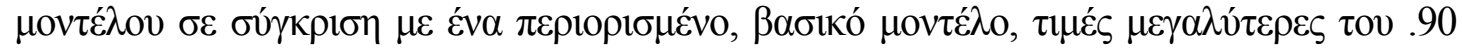

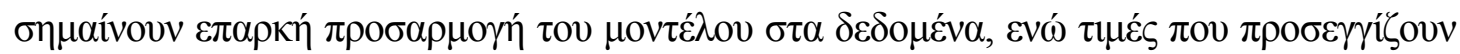

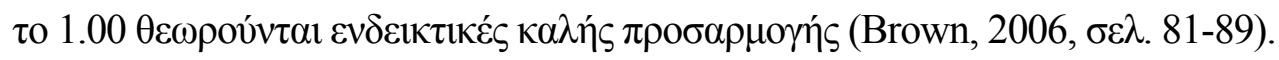

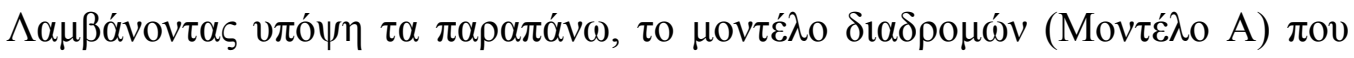

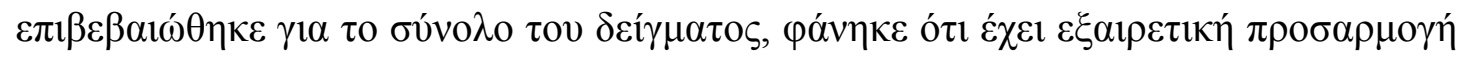

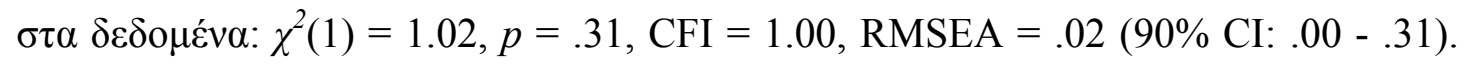




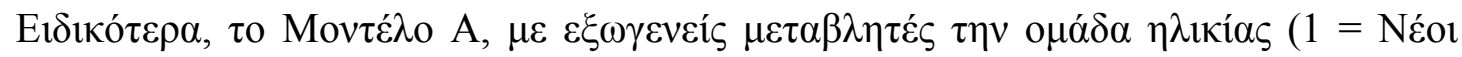

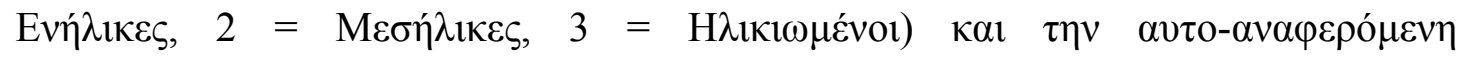

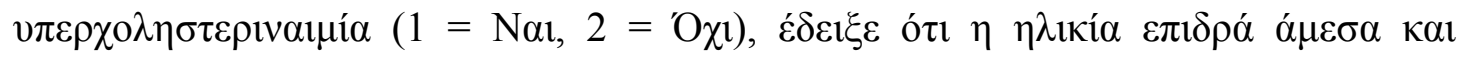

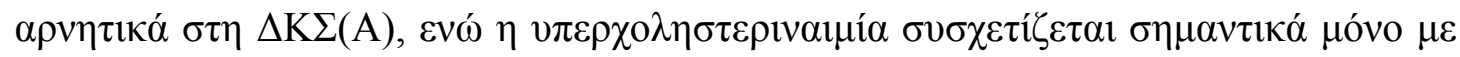

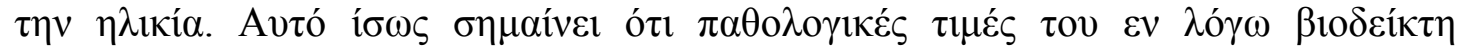

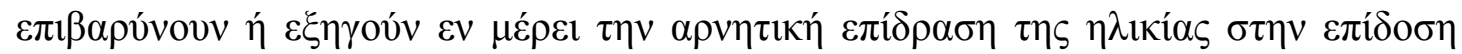
$\sigma \tau \eta \Delta \mathrm{K} \Sigma(\mathrm{A})(\beta \lambda . \Sigma \chi \eta \dot{\mu \alpha} 1)$.

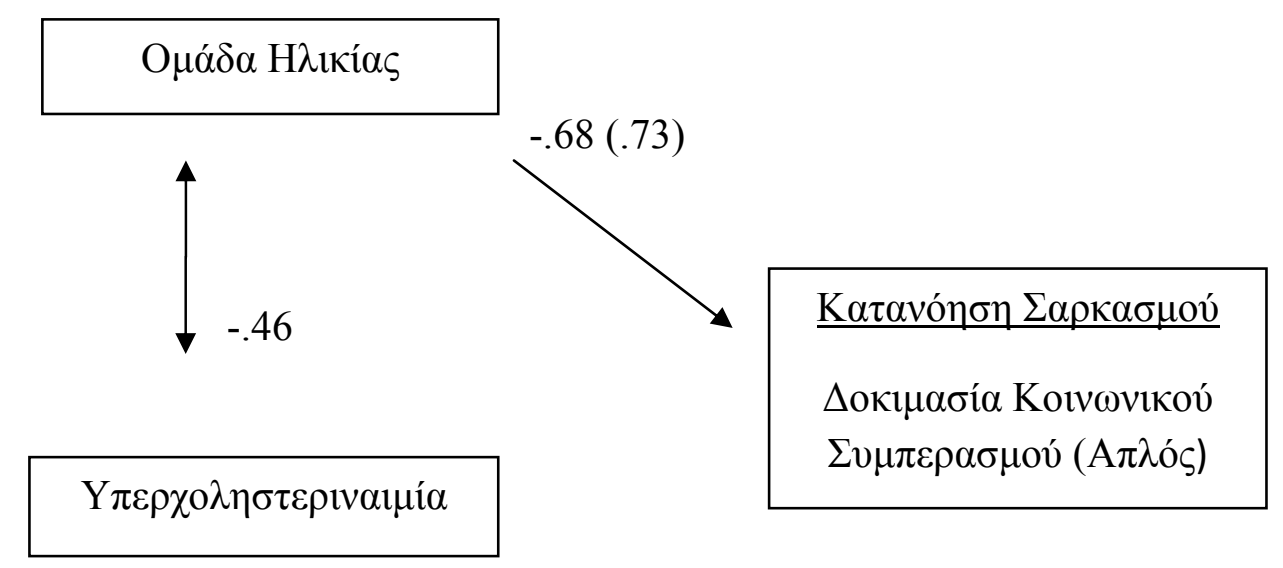

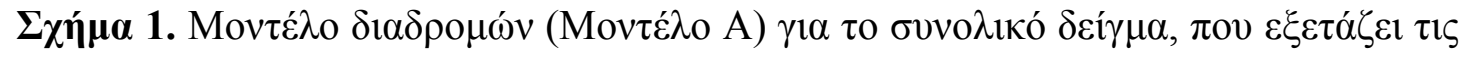

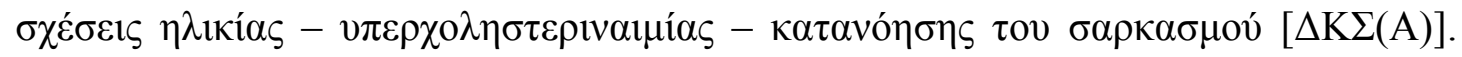

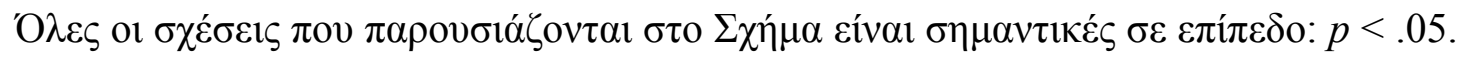

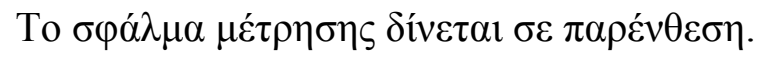

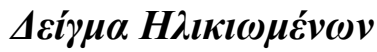

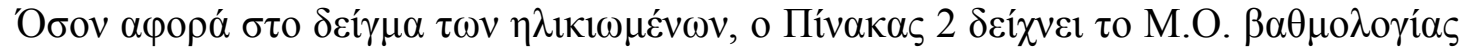

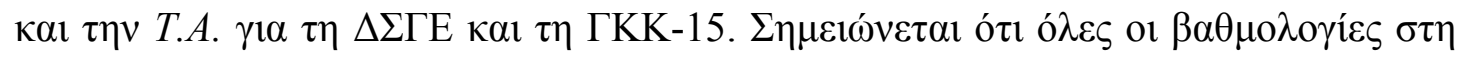

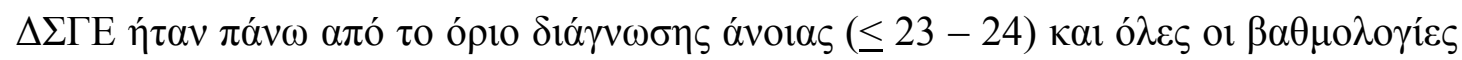

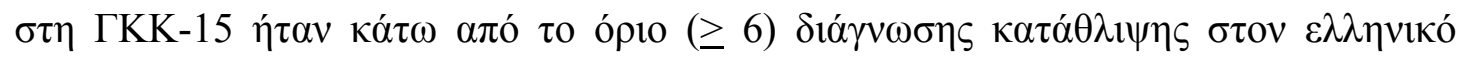
$\pi \lambda \eta \theta v \sigma \mu o^{\prime}$ 


\section{Пívakas 2}

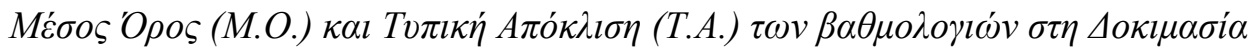

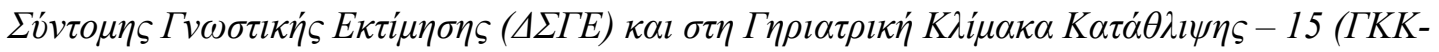

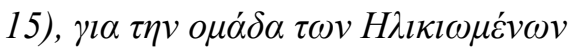

\begin{tabular}{|c|c|c|c|c|c|}
\hline & $N$ & $\mathrm{E} \lambda \alpha ́ \chi \chi 1 \sigma \tau o$ & Mغ́ $\gamma 1 \sigma \tau о$ & M.O. & T. $A$ \\
\hline$\Delta \Sigma \Gamma \mathrm{E}$ & 24 & 27 & 30 & 28.9 & 1.0 \\
\hline ГКК-15 & 24 & 1 & 4 & 2.2 & 1.0 \\
\hline
\end{tabular}

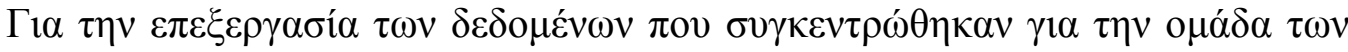

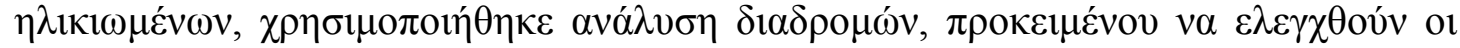

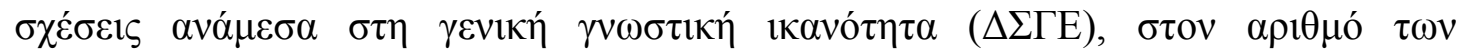

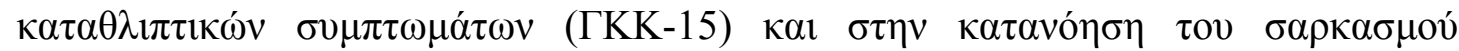

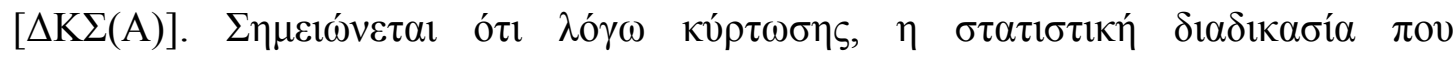

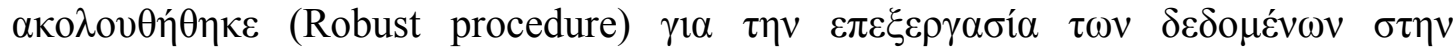

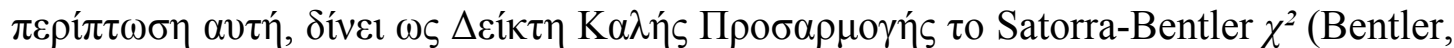

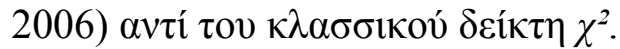

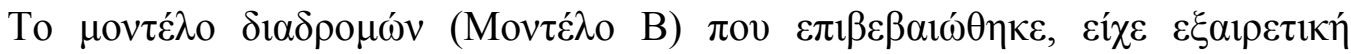

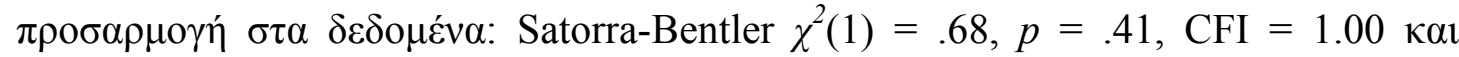

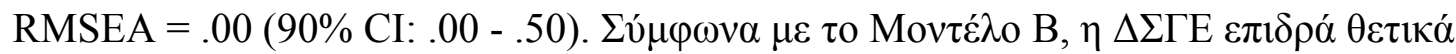

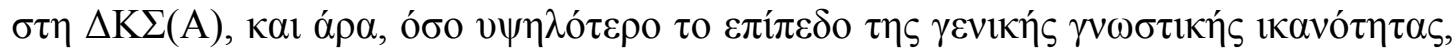

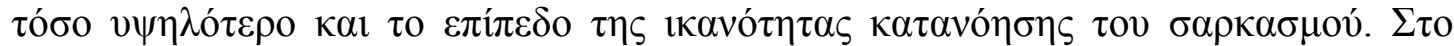

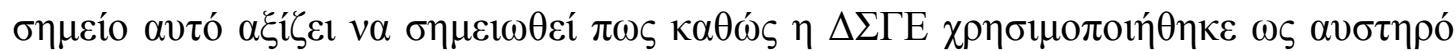

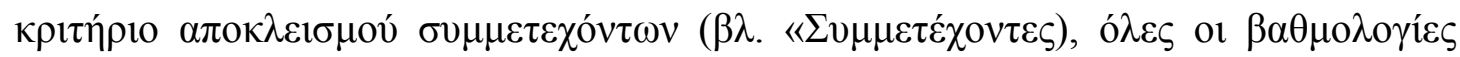

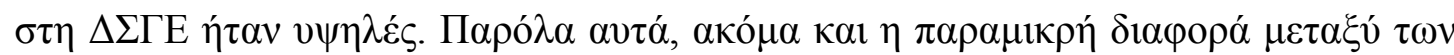

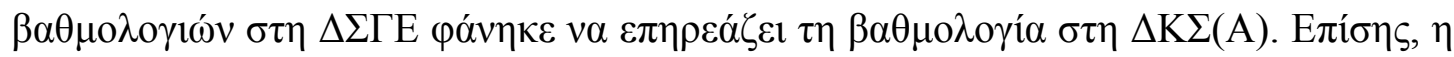




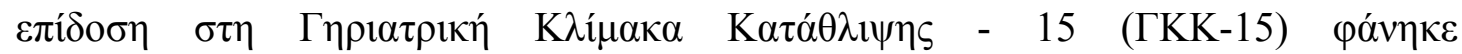

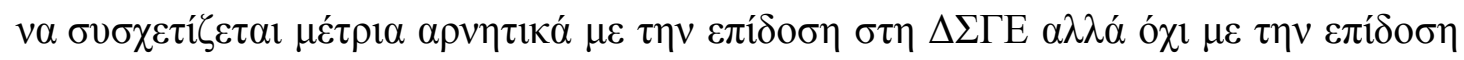

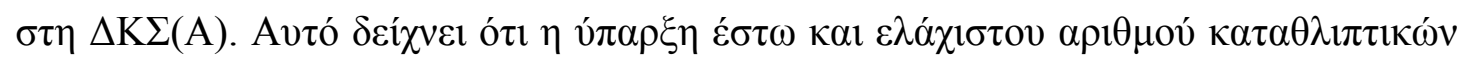

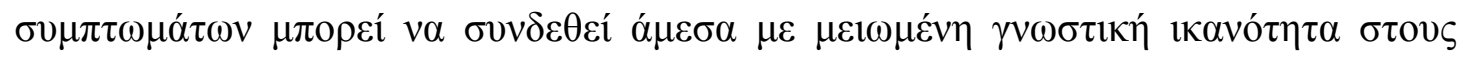

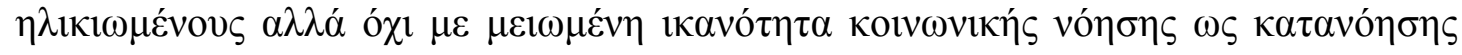

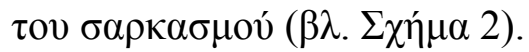

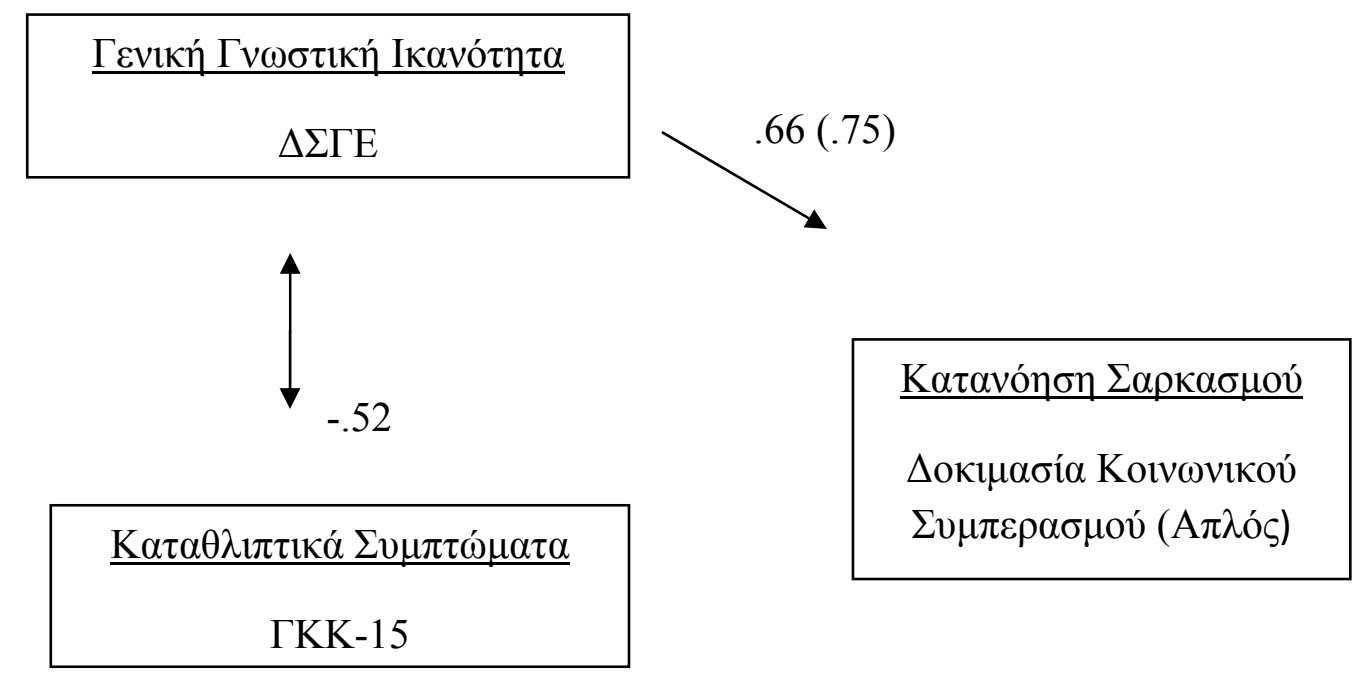

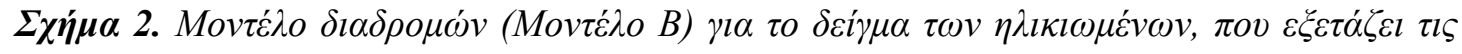

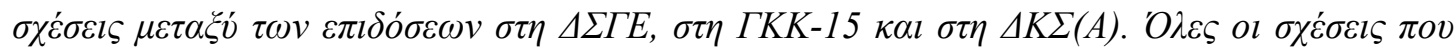

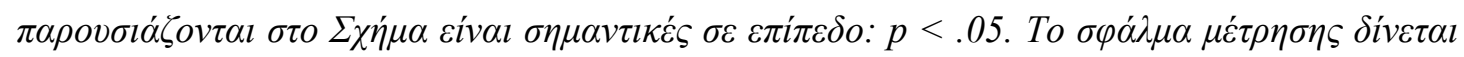
$\sigma \varepsilon \pi \alpha \rho \varepsilon ́ v \theta \varepsilon \sigma \eta$. 


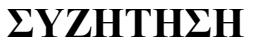

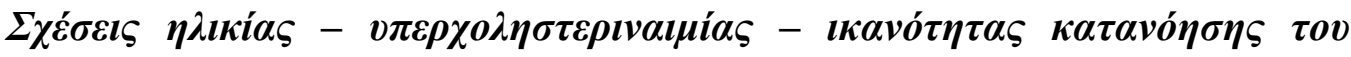 $\sigma \alpha \rho \kappa \alpha \sigma \mu o v$}

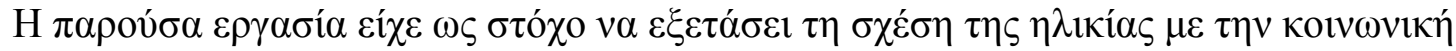

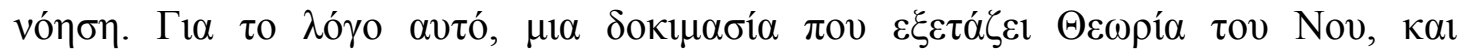

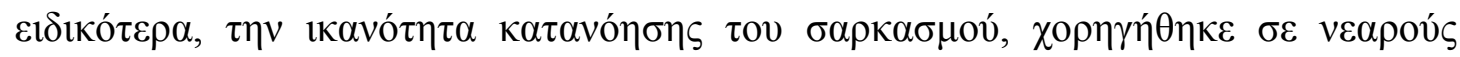

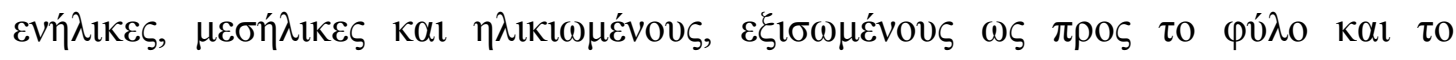

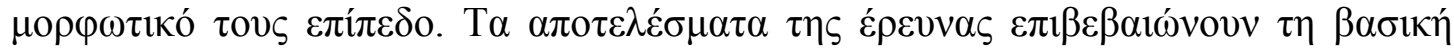

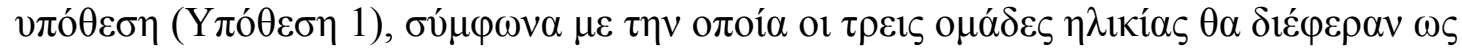

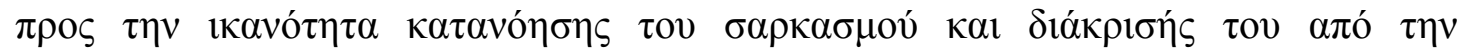

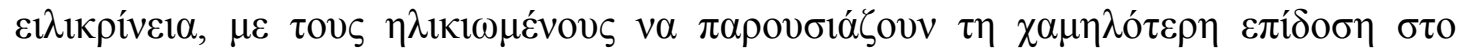

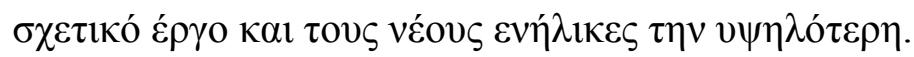

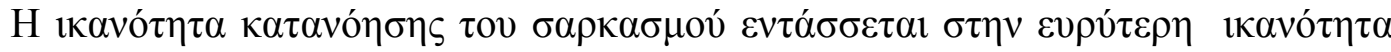

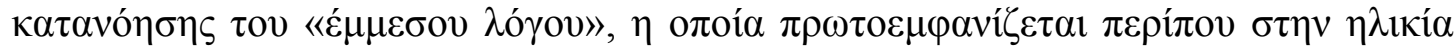

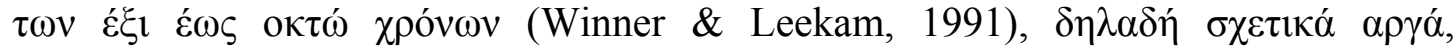

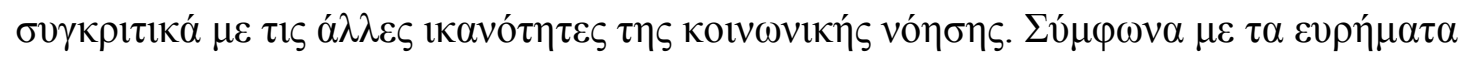

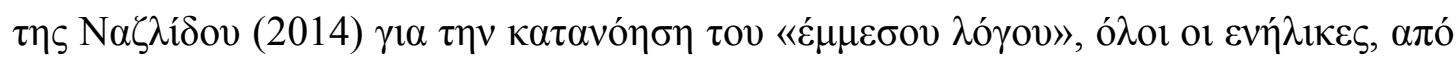

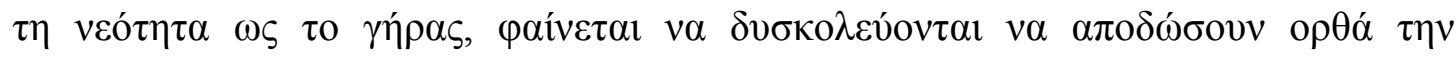

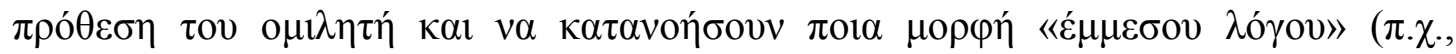

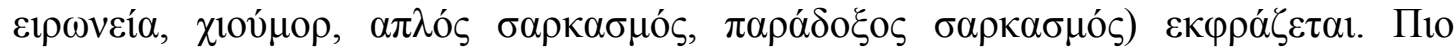

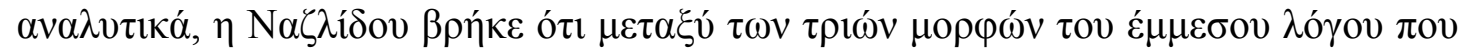

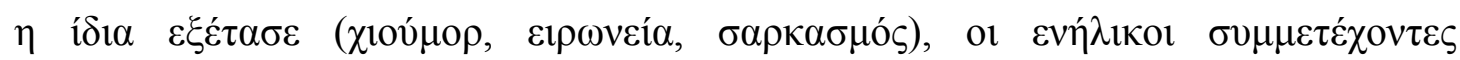

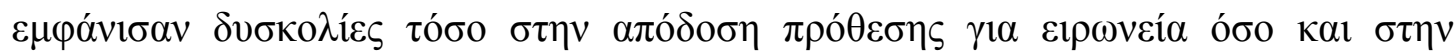

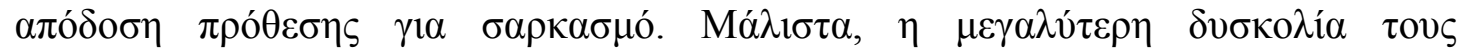




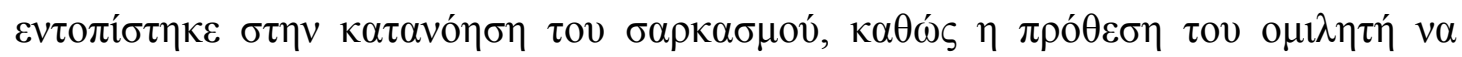

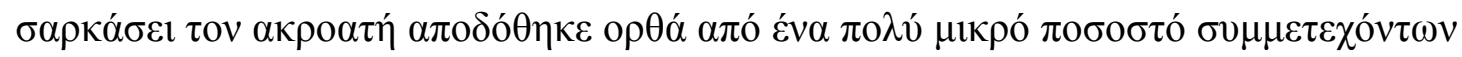

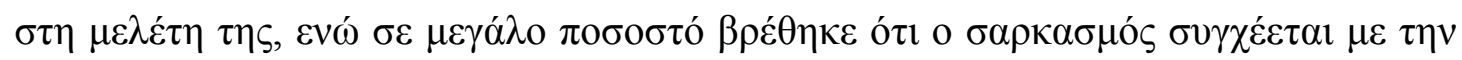

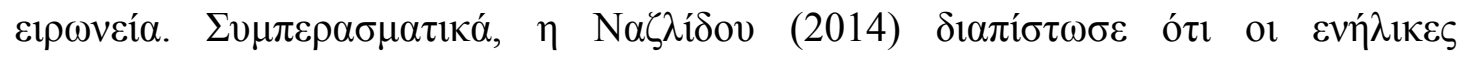

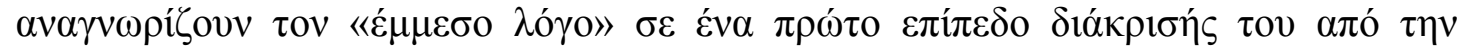

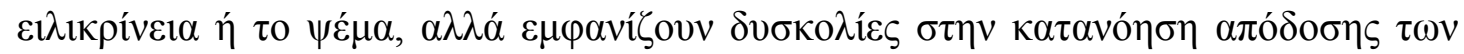

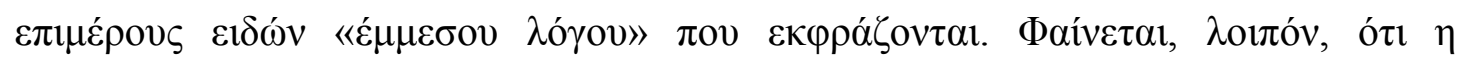

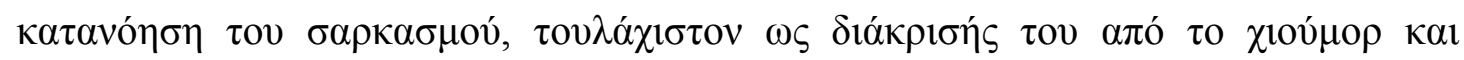

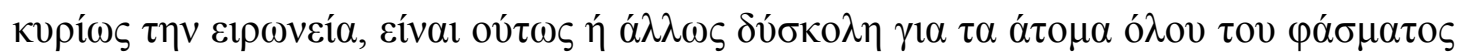

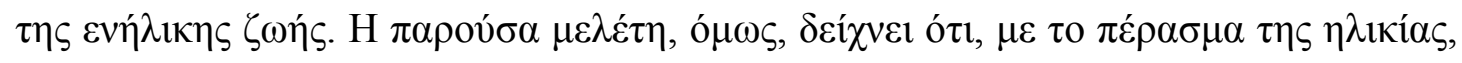

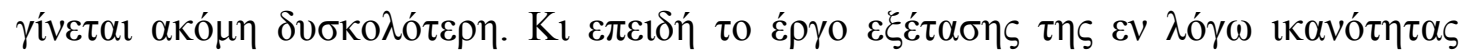

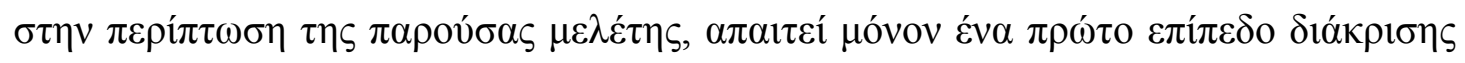

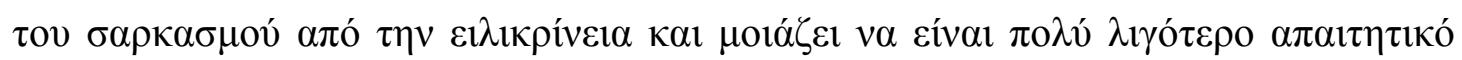

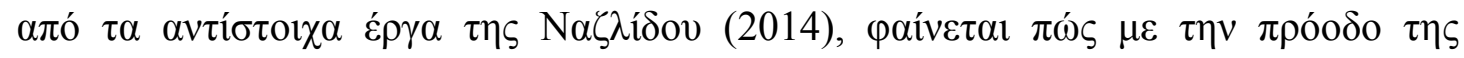

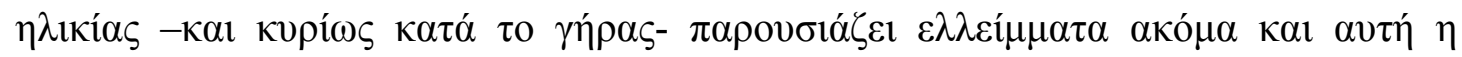

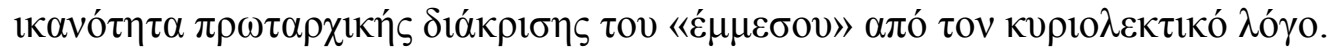

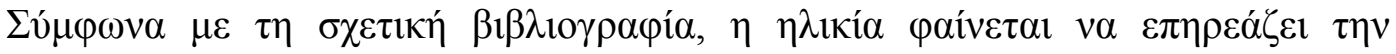

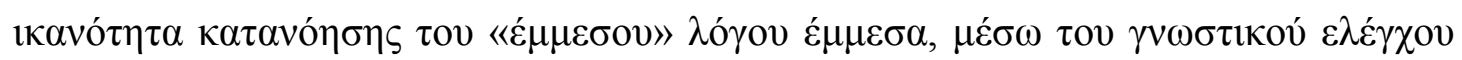

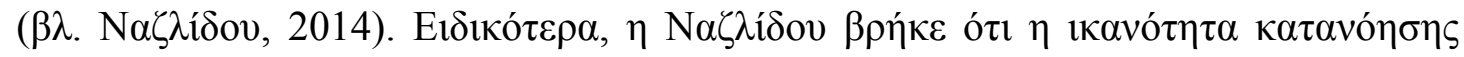

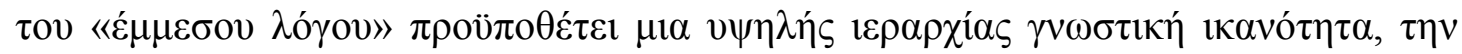

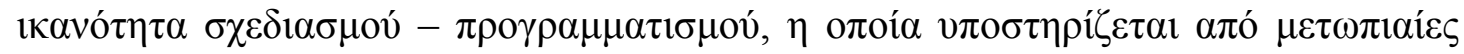

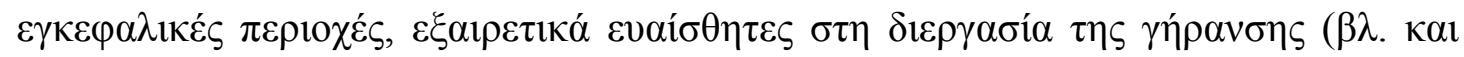

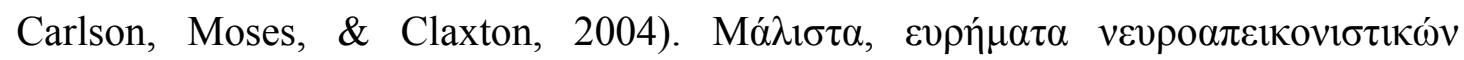

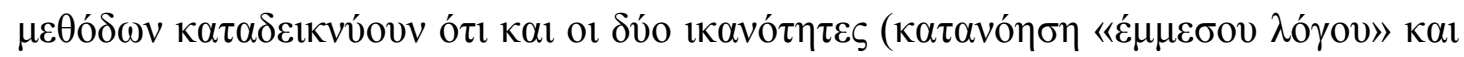

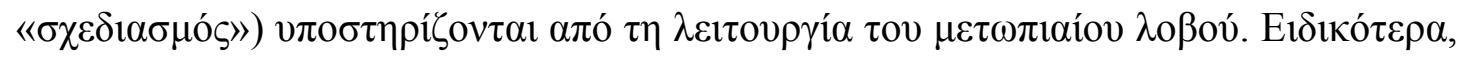




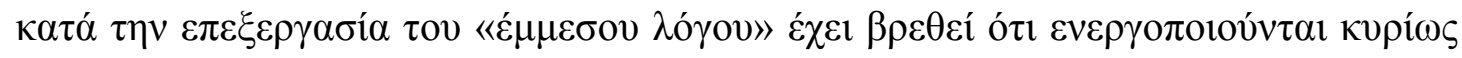

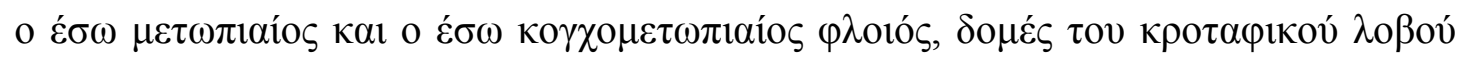

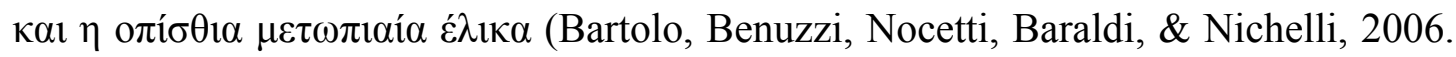

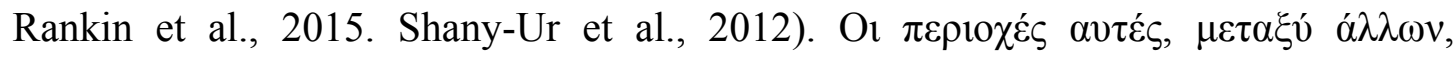

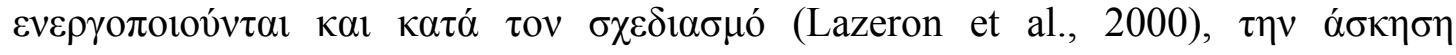

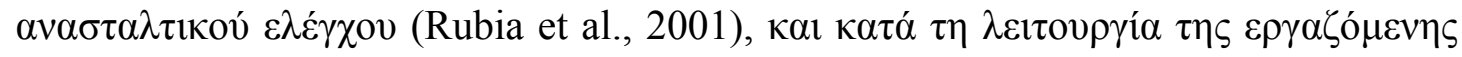

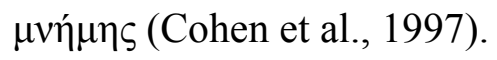

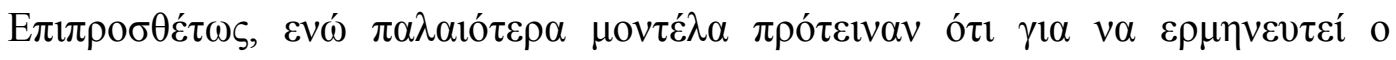

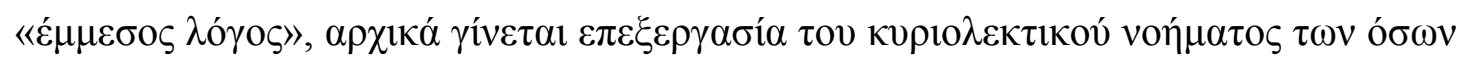

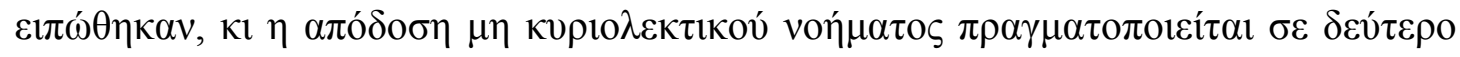

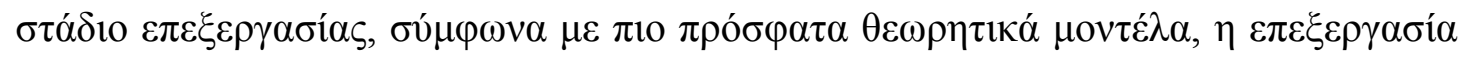

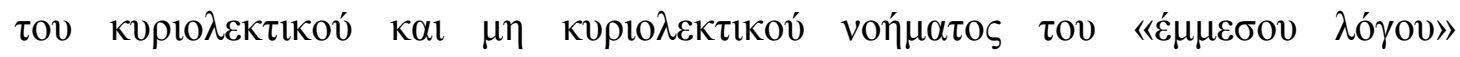

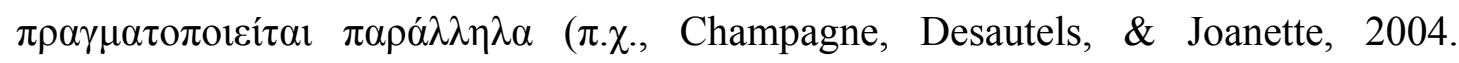
Gernsbacher, Keysar, Robertson, \& Werner, 2001). $\Sigma \tau \eta \nu ~ \tau \varepsilon \lambda \varepsilon v \tau \alpha i ́ \alpha ~ \pi \varepsilon \rho i ́ \pi \tau \omega \sigma \eta$

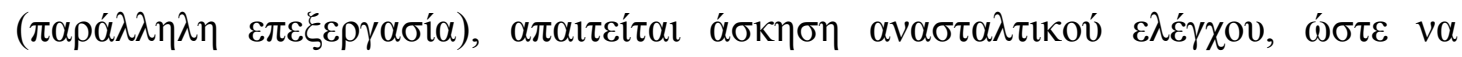

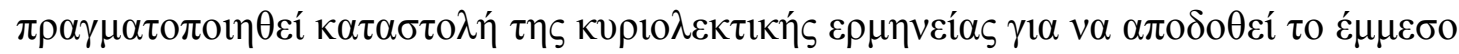

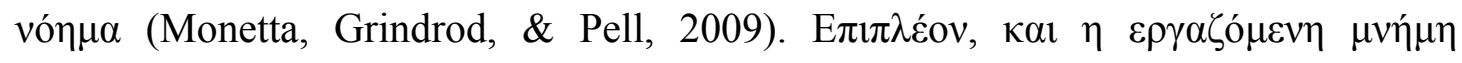

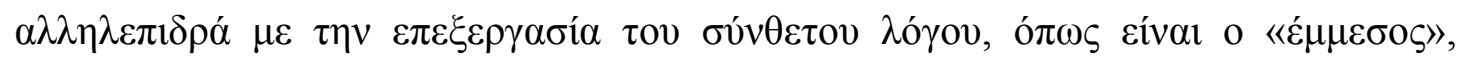

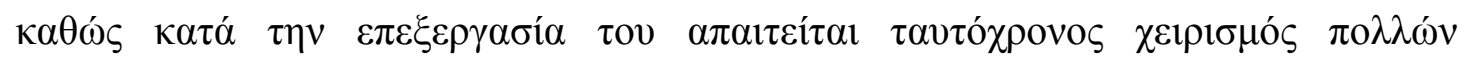

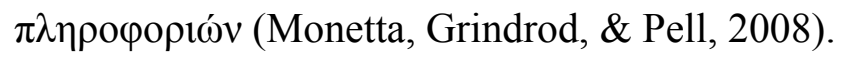

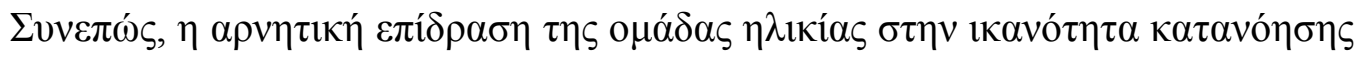

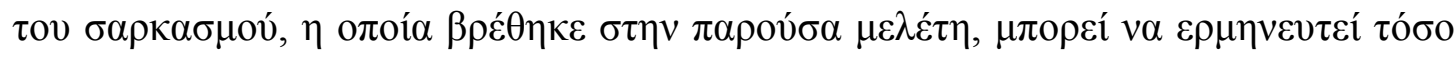

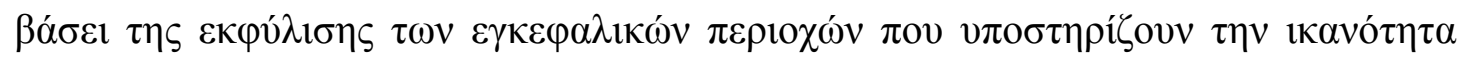

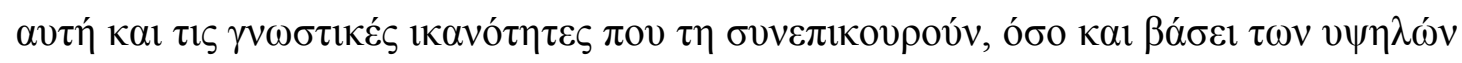

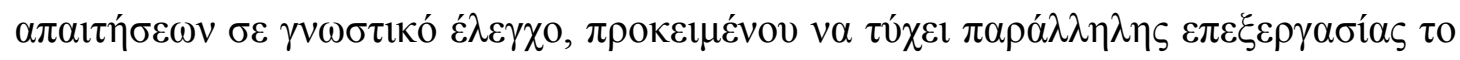




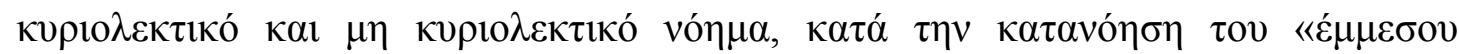

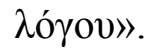

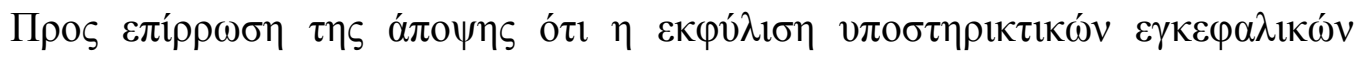

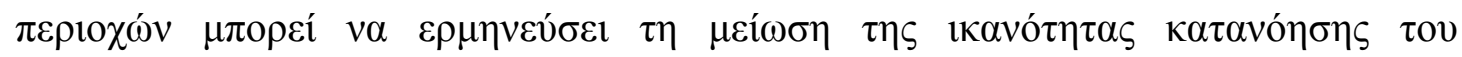

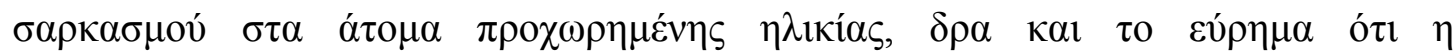

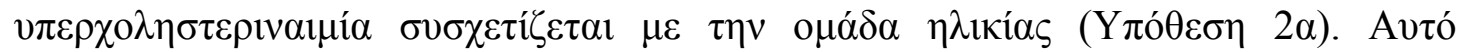

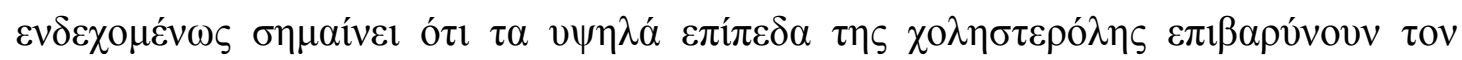

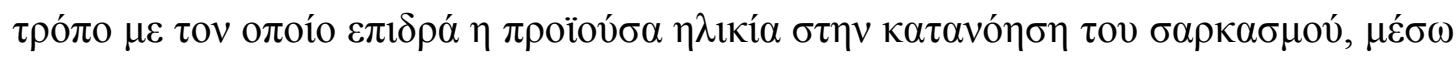

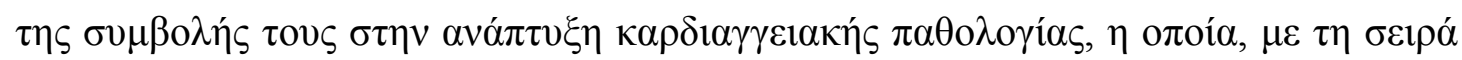

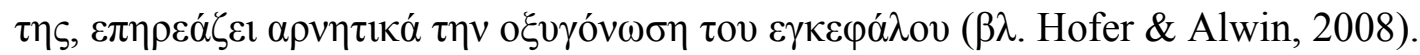

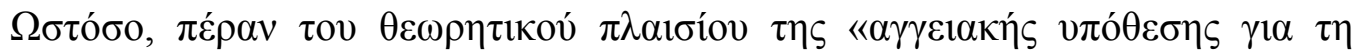

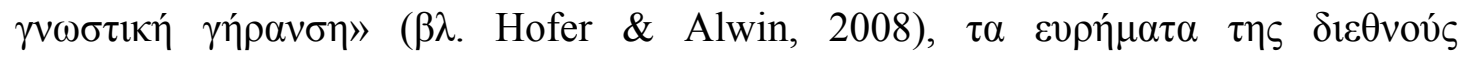

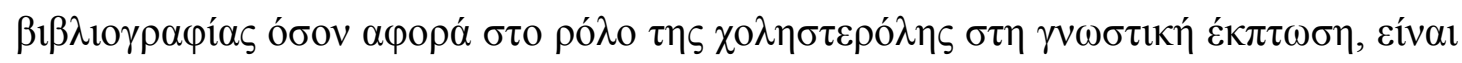

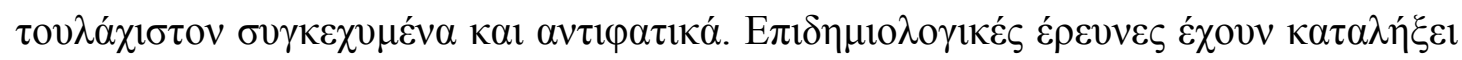

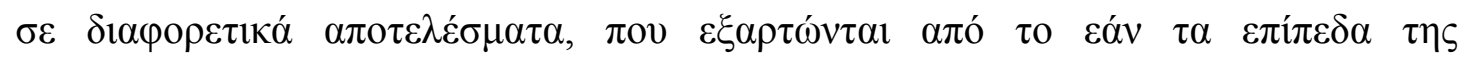

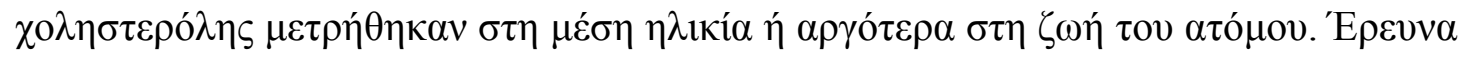

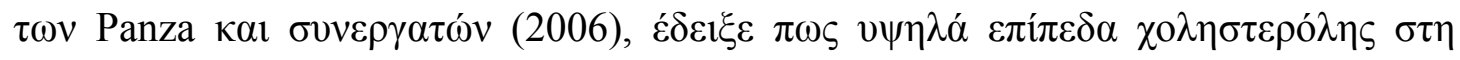

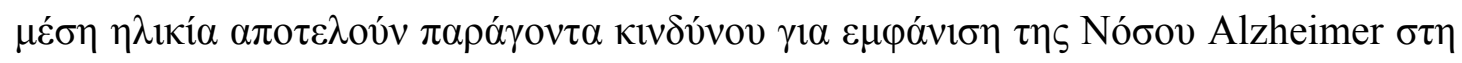

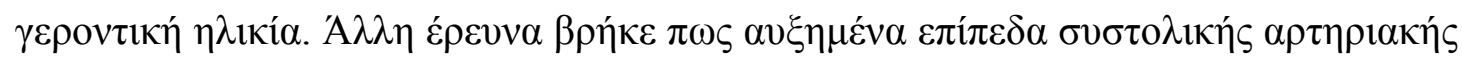

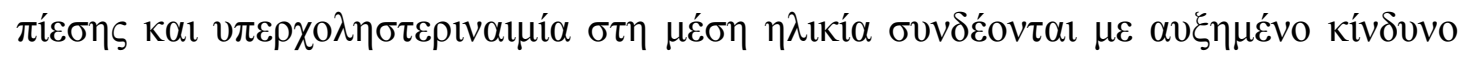

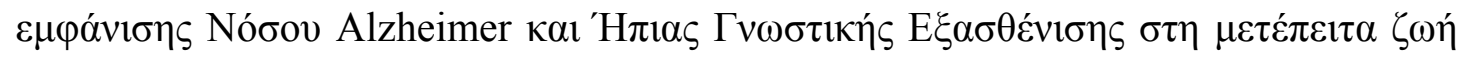

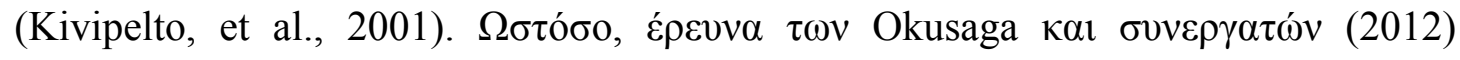

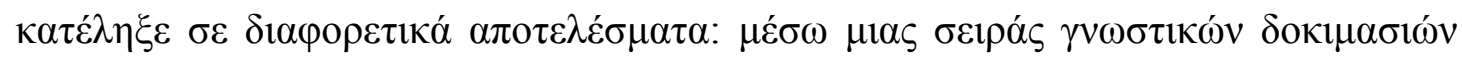

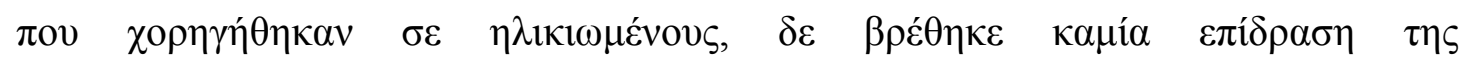

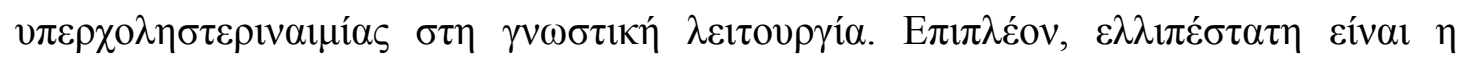




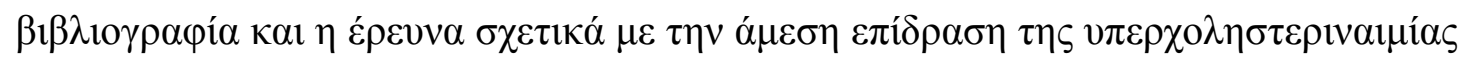

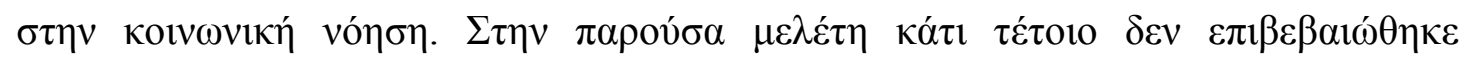

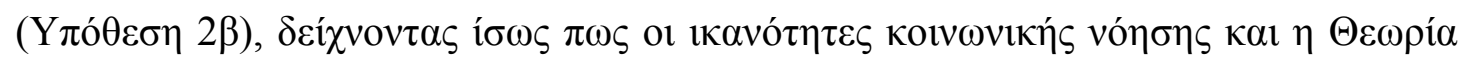

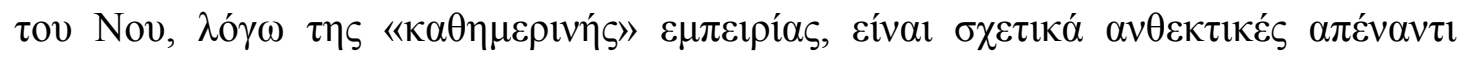

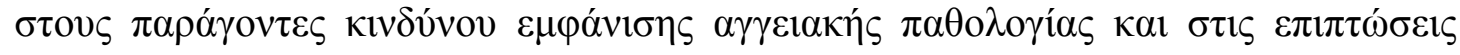

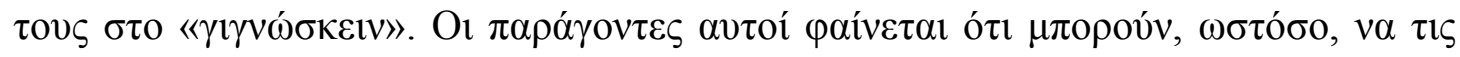

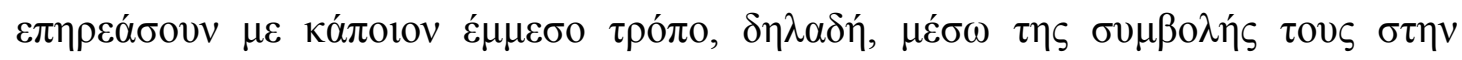

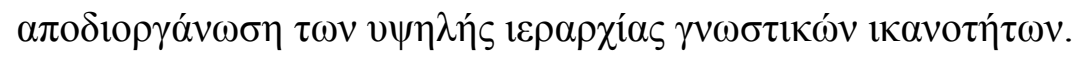

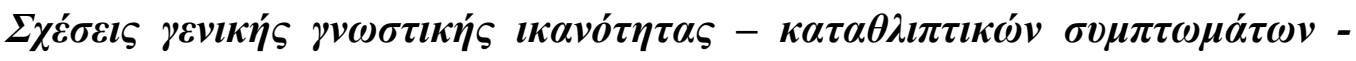

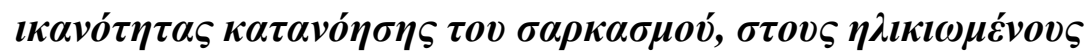

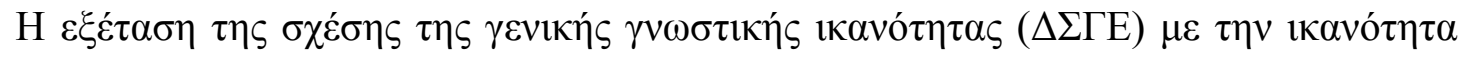

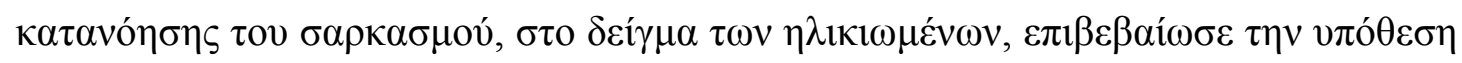

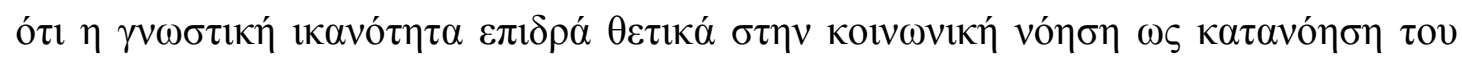

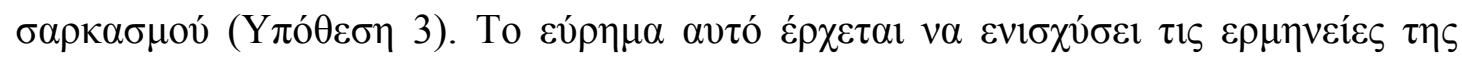

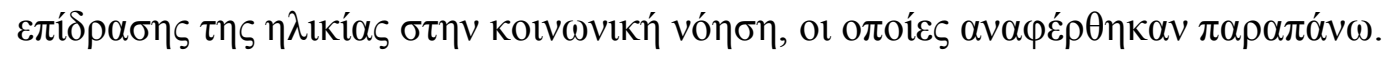

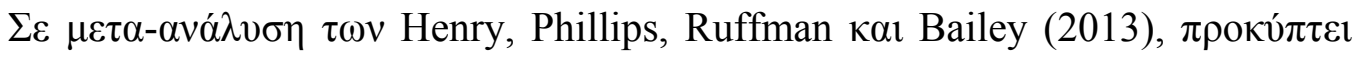

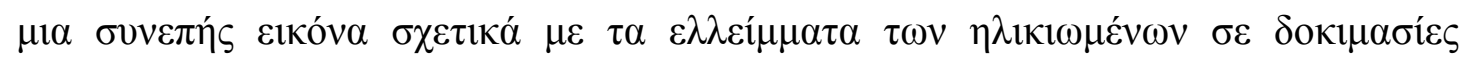

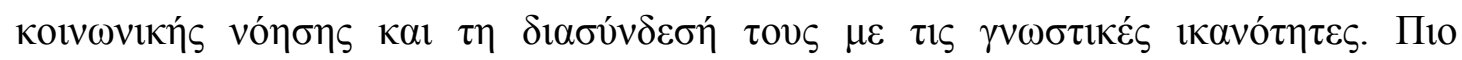

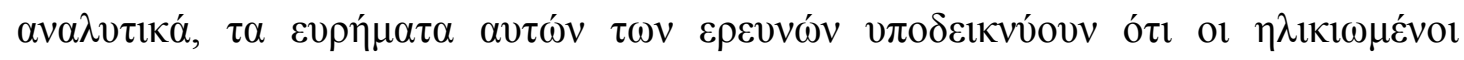

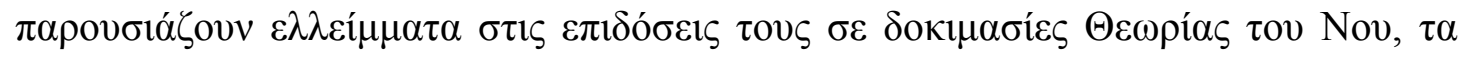

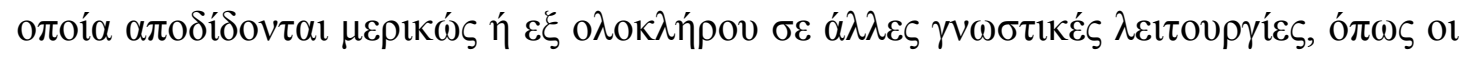

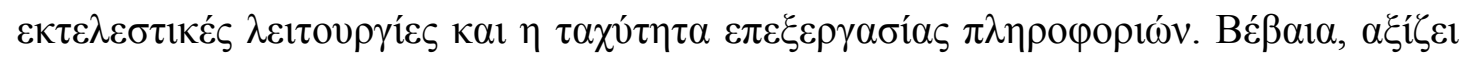

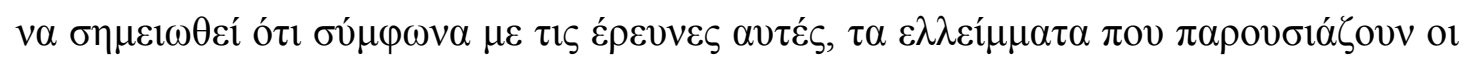

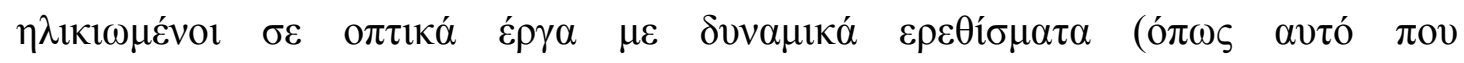




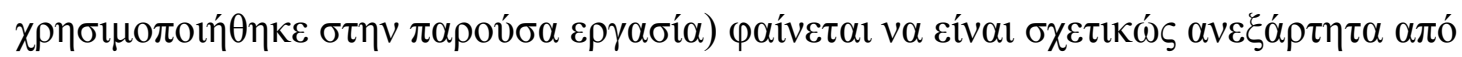

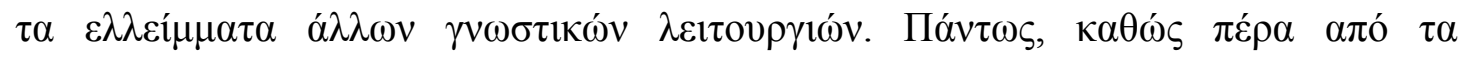

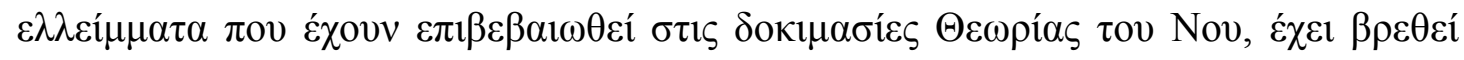

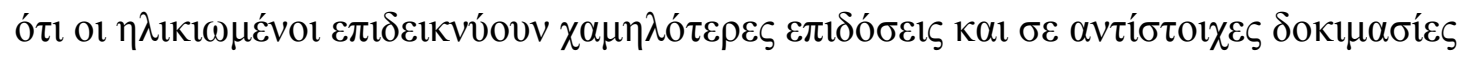

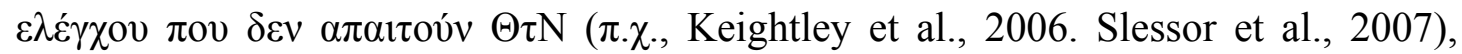

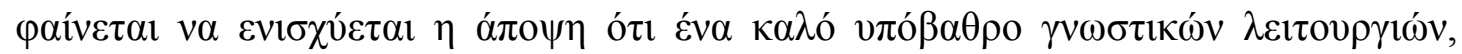

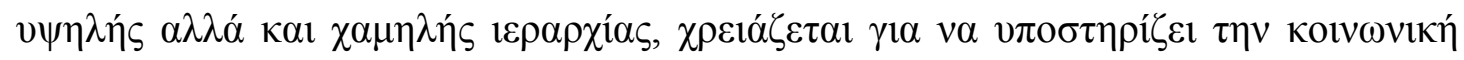

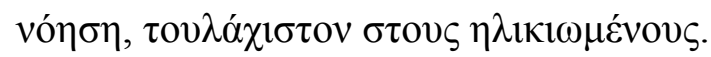

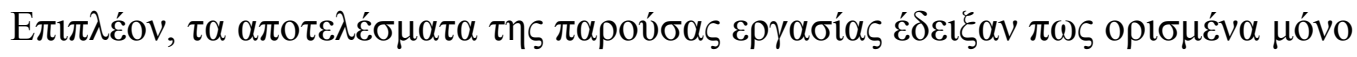

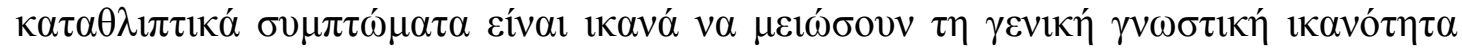

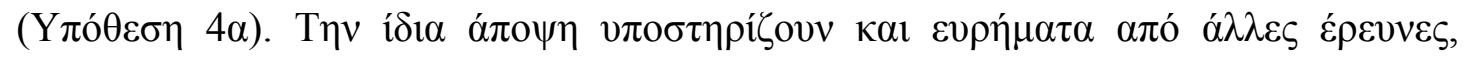

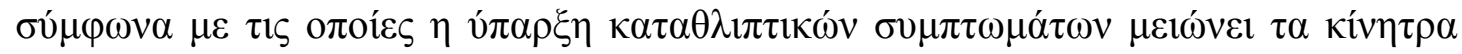

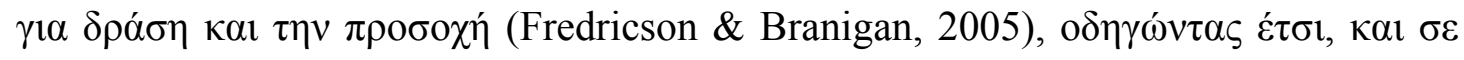

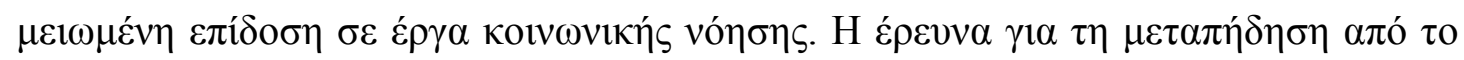

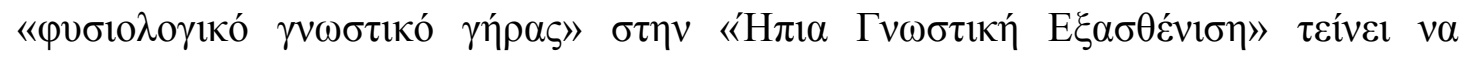

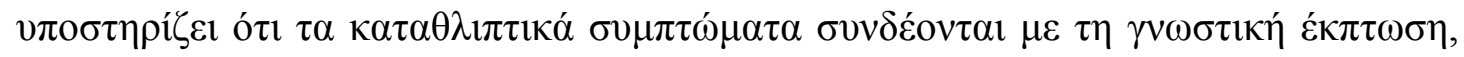

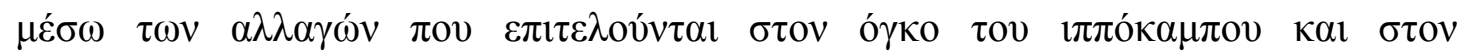

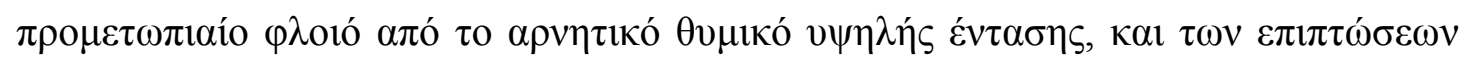

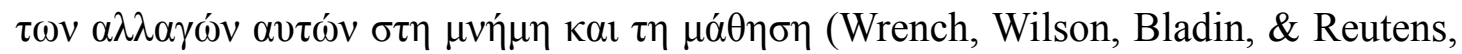

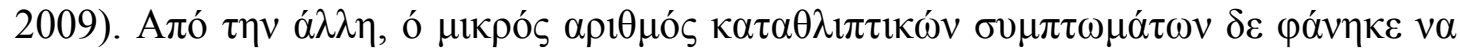

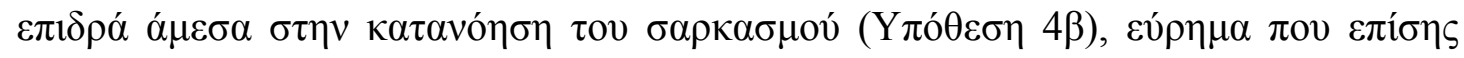

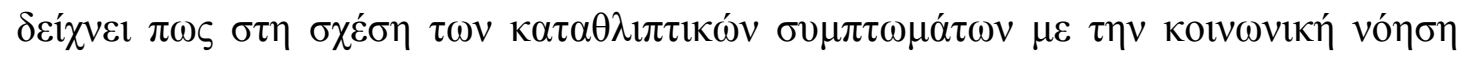

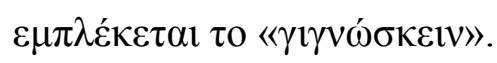




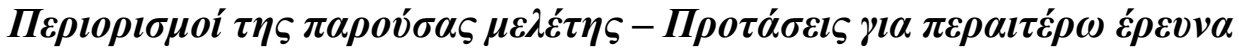

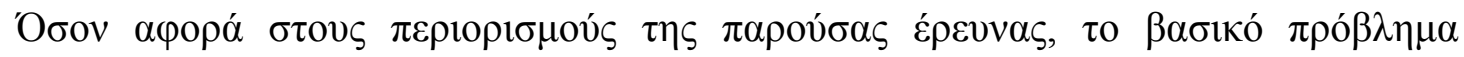

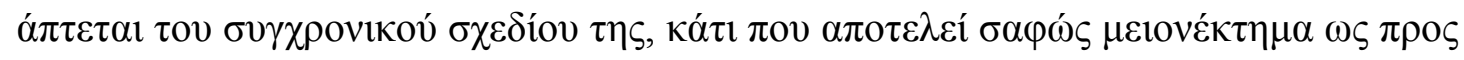

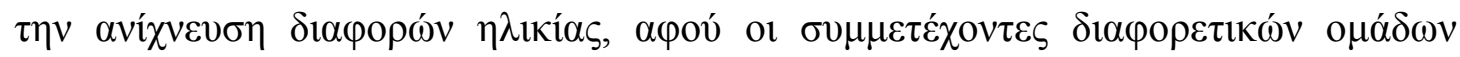

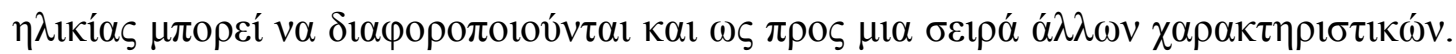

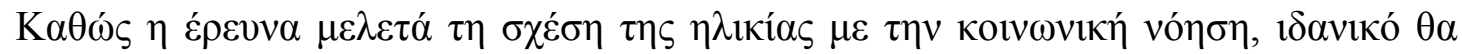

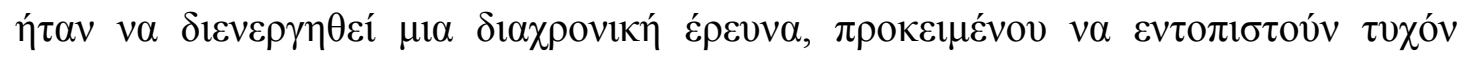

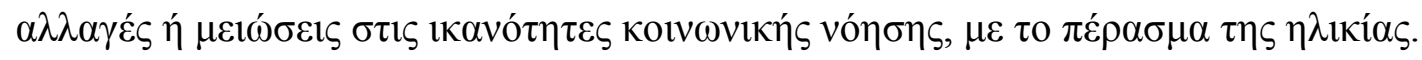

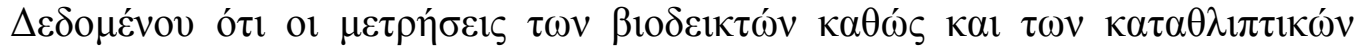

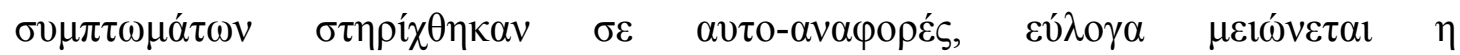

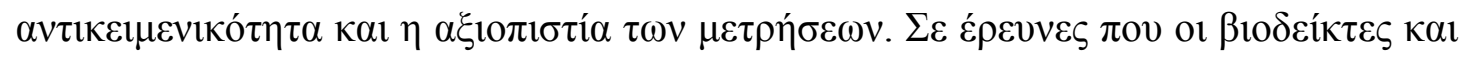

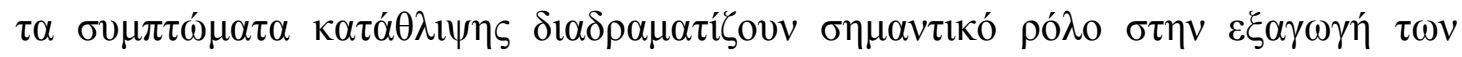

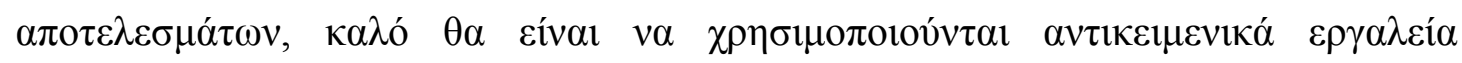

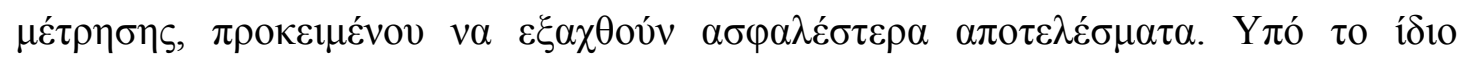

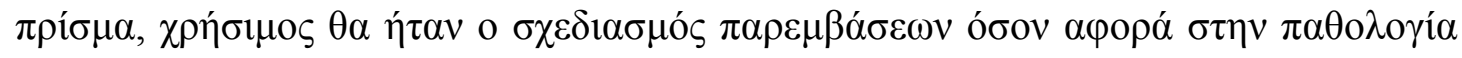

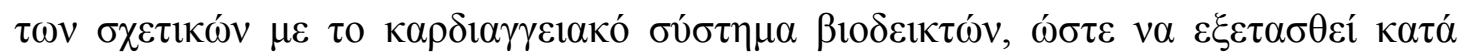

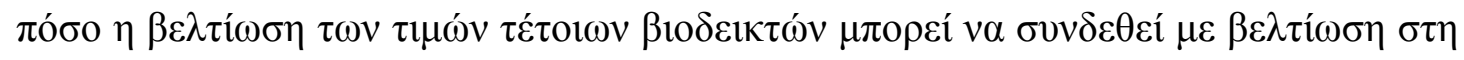

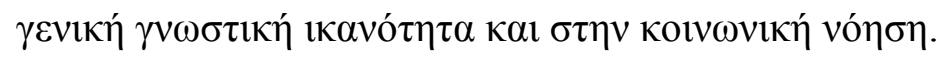

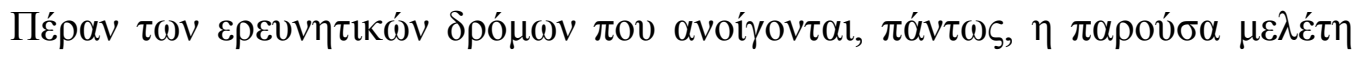

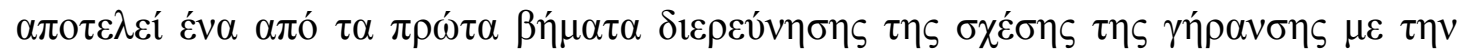

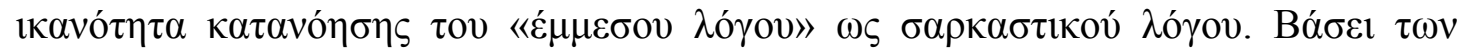

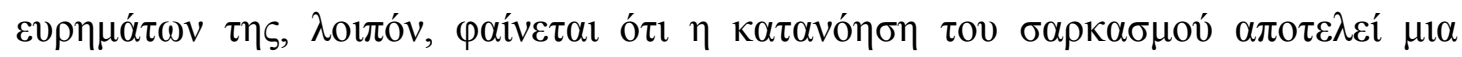

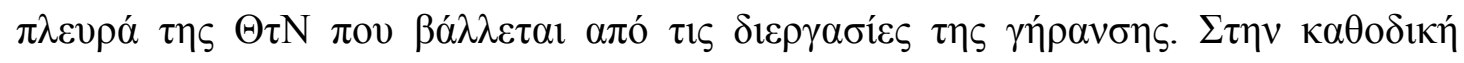

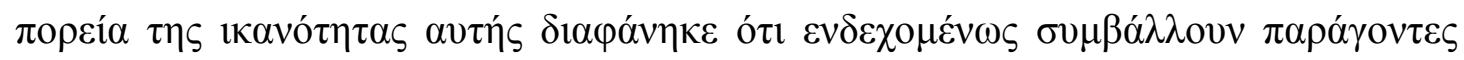

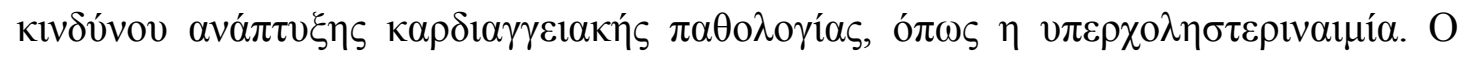




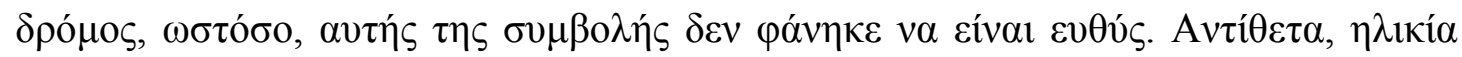

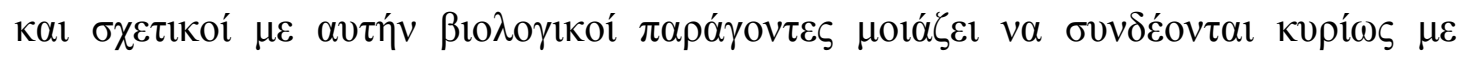

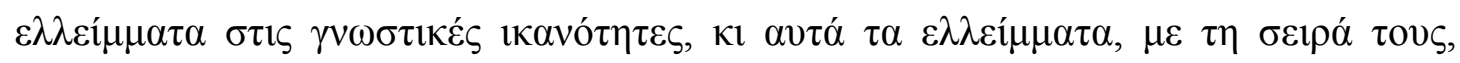

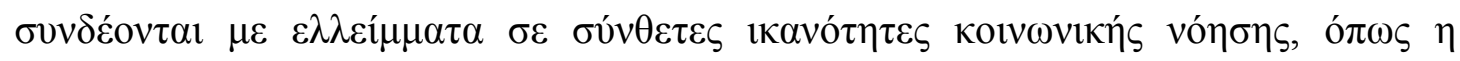

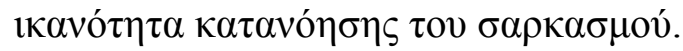




\section{ВIВАІОГРАФІА}

Adolphs, R. (2001). The neurobiology of social cognition. Current Opinion in Neurobiology, 11, 231-239.

Almeida, O. P., \& Almeida, S. A. (1999). Short versions of the Geriatric Depression Scale: a study of their validity for the diagnosis of a major depressive episode according to ICD-10 and DSM-IV. International Journal of Geriatric Psychiatry, 14, 858-865.

Anstey, K. (2008). Cognitive aging and functional biomarkers. In S. Hofer and D. Alwin (Eds.), Handbook of cognitive aging: interdisciplinary perspectives (pp. 327-339). Thousand Oaks, California: Sage.

Angeleri, R., \& Airenti, G. (2014). The development of joke and irony understanding: A study with 3- to 6-year-old children. Canadian Journal of Experimental Psychology, 68(2), 133-146.

Baron-Cohen, S., Leslie, A. M., \& Frith, U. (1985). Does the autistic child have a "theory of mind"? Cognition, 21(1), 37-46.

Bartolo, A., Benuzzi, F., Nocetti, L., Baraldi, P., \& Nichelli, P. (2006). Humor comprehension and appreciation: an FMRI study. Journal of Cognitive Neuroscience, 18(11), 1789-98.

Beaudreau, S. A., \& O'Hara, R. (2009). The association of anxiety and depressive symptoms with cognitive performance in community-dwelling older adults. Psychology and Aging, 24(2), 507-512.

Bentler, P. (2006). EQS 6 Structural equations program manual. Encino, CA: Multivariate Software, Inc. 
Bernstein, D. M., Thornton, W. L., \& Sommerville, J. A. (2011). Theory of Mind through the ages: older and middle-aged adults exhibit more errors than do younger adults on a continuous false belief task. Experimental Aging Research, 37(5), 481-502.

Blakemore, S., \& Choudhury, S. (2006). Development of the adolescent brain: implications for executive function and social cognition. Journal of Child Psychology and Psychiatry, 47(3-4), 296-312.

Brothers, L. (1990). The social brain: A project for integrating primate behavior and neurophysiology in a new domain. Concepts in Neuroscience, 1, 27-61.

Brown, T. A. (2006). Confirmatory factor analysis for applied research. New York: The Guilford Press.

Carlson, M. S., Moses, J. L., \& Claxton, J. L. (2004). Individual differences in executive functioning and Theory of Mind: An investigation of inhibitory control and planning ability. Journal of Experimental Child Psychology, 87, 299-319.

Carstensen, L., Fung, H., \& Charles, S. T. (2003). Socioemotional selectivity theory and the regulation of emotion in the second half of life. Motivation and Emotion, 27, 103-123.

Champagne, M., Desautels, M. C., \& Joanette, Y. (2004). Lack of inhibition could contribute to non-literal language impairments in right-hemisphere-damaged individuals. Brain and Language, 91(1), 172-174.

Channon, S., Pellijeff, A., \& Rule, A. (2005). Social cognition after head injury: sarcasm and theory of mind. Brain and Language, 93(2), 123-34.

Cohen, J. D., Perlstein, W. M., Braver, T. S., Nystrom, L. E., Noll, D. C., Jonides, J., \& Smith, E. E. (1997). Temporal dynamics of brain activation during a 
working memory task control through adolescence? Brain and Cognition, 72, $101-113$

Couture, M. S., Penn, L. D., \& Roberts, L. D. (2006). The functional significance of social cognition in schizophrenia: A review. Schizophrenia Bulletin, 32, 4463.

Dews, S., \& Winner, E. (1997). Attributing meaning to deliberately false utterances: The case of irony. Advances in Psychology, 122, 377-414.

Dumontheil, I., Apperly, I. A., \& Blakemore, S. (2010). Online usage of theory of mind continues to develop in late adolescence. Developmental Science, 13(2), $331-338$.

Duval, C., Piolino, P., Bejanin, A., Eustache, F., \& Desgranges, B. (2011). Age effects on different components of Theory of Mind. Conciousness and Cognition, 20(3), 627-642.

Elias, P., Elias, M. F., D’Agostino, R. B., Sullivan, L. M., \& Wolf, P. A. (2005). Serum cholesterol and cognitive performance in the Framingham Heart Study. Journal of Biobehavioral Medicine, 67(1), 24-30.

Folstein, M. F., Folstein, S. E., \& McHugh, P. R. (1975). "Mini- Mental State": a practical method for grading the cognitive state of patients for the clinician. Journal of Psychiatric Research, 12, 189-198.

Folstein, M. F., Robins, L. N., \& Helzer, J. E. (1983). The Mini-Mental State Examination. Journal of American Medical Association, 40(7), 812.

Fountoulakis, K., Tsolaki, M., Chatzi, H., \& Kazis, A. (2000). Mini Mental State Examination (MMSE): A validation study in Greece. American Journal of Alzheimer's Disease and other Dementias, 15(6), 342-345. 
Fountoulakis K. N., Tsolaki, M., Iacovides, A., et al. (1999). The validation of the short form of the Geriatric Depression Scale (GDS) in Greece. Aging, 11(6), $367-372$.

Fredricson, B. L., \& Branigan, C. (2005). Positive emotions broaden the scope of attention and through-action repertoires. Cognition and Emotion, 19(3), 313332.

Gernsbacher, M. A., Keysar, B., Robertson, R. R., \& Werner, N. K. (2001). The role of suppression and enhancement in understanding metaphors. Journal of Memory and Language, 45(3), 433-450

Grady, C. L. (2008). Cognitive neuroscience of aging. Annals of the New York Academy of Sciences, 1124, 127- 144.

Green, F. M., Olivier, B., Crawley, N. J., Penn, L. D., \& Silverstein, S. (2005). Social cognition in schizophrenia: Recommendations from the Measurement and Treatment Research to Improve Cognition in Schizophrenia New Approaches Conference. Schizophrenia Bulletin, 31(4), 882-887.

Happe, F. G. E., Winner, E., \& Brownell, H. (1998). The getting of wisdom: Theory of Mind in old age. Developmental Psychology, 34(2), 358-362.

Harada, T., Itakura, S., Xu, F., Lee, K., Nakashita, S., Saito, D. N., \& Sadato, N. (2009). Neural correlates of the judgment of lying: A functional magnetic resonance imaging study. Neuroscience Research, 63(1), 24-34.

Henry, J. D., Phillips, L. H., Ruffman, T., \& Bailey, P. E. (2013). A meta-analytic review of age differences in Theory of Mind. Psychology and Aging, 28(3), 826-839.

Hofer, S., \& Alwin, D. (Eds.) (2008). Handbook of cognitive aging: interdisciplinary perspectives. Thousand Oaks, California: Sage. 
Keightley, M. L., Winocur, G., Burianova, H., Hongwanishkul, D., \& Grady, C. L. (2006). Age effects on social cognition: faces tell a different story. Psychology and Aging, 21(3), 558-572.

Kivipelto, M., Helkala, E. L., Laakso, M. P., Hänninen, T., Hallikainen, M., Alhainen, K., Soininen, H., Tuomilehto, J., \& Nissinen, A. (2001). Midlife vascular risk factors and Alzheimer's disease in later life: longitudinal, population based study. British Medical Journal, 322(7300), 1447-1451.

Kryla-Lighthall, N., \& Mather, M. (2009). The role of cognitive control in older adults' emotional well-being. In V. Bengston, D. Gans, N. Putney and M. Silverstein (Eds.), Handbook of theories of aging (pp. 323-344). New York: Springer.

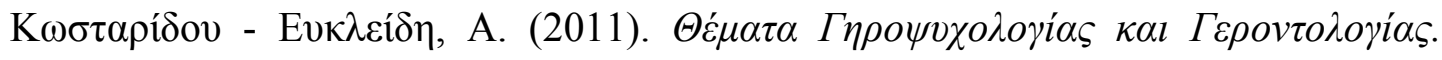

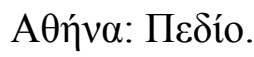

Lautenschlager, N. T., Cox, K. L., Flicker, L. F., Foster, J. K., Van Bockxmeer, F. M., Xiao, J., Greenop, K. R., \& Almeida, O. P. (2008). Effect of physical activity on cognitive function in older adults at risk for Alzheimer disease. A randomized trial. Journal of American Medical Association, 300, 1027-1037.

Lazeron, R. H., Rombouts, S. A., Machielsen, W. C., Scheltens, P., Witter, M. P., Uylings, H. B., \& Barkhof, F. (2000). Visualizing brain activation during planning: the tower of London test adapted for functional MR imaging. American Journal of Neuroradiology, 21(8), 1407-1414.

Leslie, A. M. (1987). Pretence and representation: The origins of "theory of mind." Psychological Review, 94, 412-426. 
Lough, S., Gregory, C., \& Hodges, J. R. (2001). Dissociation of social cognition and executive function in frontal variant frontotemporal dementia. Neurocase, 7, $123-130$.

Martín-Rodríguez, J. F., \& León-Carrión, J. (2010). Theory of Mind deficits in patients with acquired brain injury: a quantitative review. Neuropsychologia, 48(5), 1181-1191.

McDonald, S., Bornhofen, C., Shum, D., Long, E., Saunders, C., \& Neulinger, K. (2006) Reliability and validity of 'The Awareness of Social Inference Test' (TASIT): A clinical test of social perception. Disability and Rehabilitation, 28(24), 1529-1542.

McDonald, S., Flanagan, S., Rollins, J., \& Kinch, J. (2003). TASIT: A new clinical tool for assessing social perception after traumatic brain injury. Journal of Head Trauma Rehabilitation, 18(3), 219-238.

Mitchell, A. J. (2009). A meta-analysis of the accuracy of the Mini-Mental State Examination in the detection of dementia and mild cognitive impairment. Journal of Psychiatric Research, 43(4), 411-431.

Monetta, L., Grindrod, C. M., \& Pell, M. D. (2008). Effects of working memory capacity on inference generation during story comprehension in adults with Parkinson's disease. Journal of Neurolinguistics, 21(5), 400-417.

Monetta, L., Grindrod, C. M., \& Pell, M. D. (2009). Irony comprehension and theory of mind deficits in patients with Parkinson's disease. Cortex, 45(8), 972-981.

Moraitou, D., \& Efklides, A. (2007). Affect and emotions. In R. FernandezBallesteros (Ed.), Geropsychology. European perspectives for an ageing world (pp. 82- 102). Goettingen, Germany: Hogrefe \& Huber. 
Moraitou, D., \& Efklides, A. (2012). The wise thinking and acting questionnaire: The cognitive facet of wisdom and its relation with memory, affect, and hope. Journal of Happiness Studies, 13(5), 849-873.

Moraitou, D., \& Papantoniou, G. (2012). Mindfulness and action control in young, middle-aged, and old adults: An examination of their relationship to attentional bias towards emotional information. Research in Psychology (Ricerche di Psicologia), 2010(4), 591-626.

Moraitou, D., Papantoniou, G., Gkinopoulos, T., \& Nigritinou, M. (2013). Older adults' decoding of emotions: Age-related differences in interpreting dynamic emotional displays and the well-preserved ability to recognize happiness. Psychogeriatrics, 13(3), 139-147.

Morales, L. S., Flowers, C., Gutierrez, P., Kleinman, M., \& Teresi, J. A. (2006). Item and scale differential functioning of the Mini-Mental State Exam assessed using the differential item and test functioning (DFIT) framework. Medical Care, 44, 143-151.

Moran, J. M. (2013). Lifespan development: the effects of typical aging on Theory of Mind. Behavioral and Brain Research,15(237), 32-40.

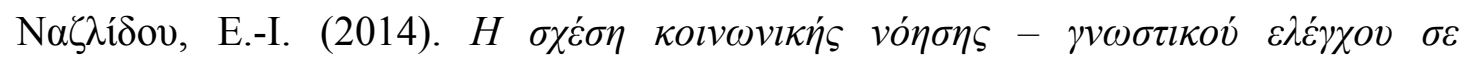

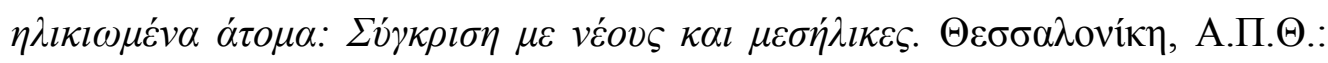

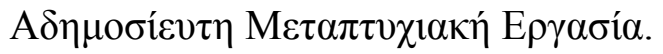

Nyunt, M. S., Fones, C., \& Niti, M. (2009). Criterion-based validity and reliability of the Geriatric Depression Screening Scale (GDS-15) in a large validation sample of community-living Asian older adults. Aging \& Mental Health, 13(3), 376- 82. 
O’ Connor, D. W., Pollitt, P. A., Hyde, J. B., Fellows, J. L., Miller, N. D., Brook, C. P. B., \& Reiss, B. B. (1989). The reliability and validity of the Mini-Mental State Examination in a British community survey. Journal of Psychiatric Research, 23(1), 87-96.

Okusaga, O., Stewart, M. C. W., Butcher, I., Deary, I., Fowkes, F. G. R., \& Price, J. F. (2012). Smoking, hypercholesterolaemia and hypertension as risk factors for cognitive impairment in older adults. Age and Ageing, 42(3), 306-311.

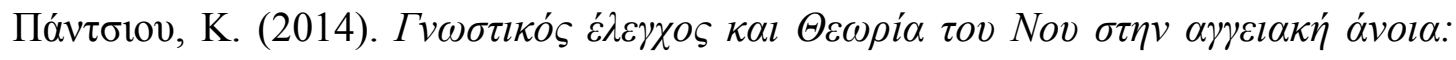

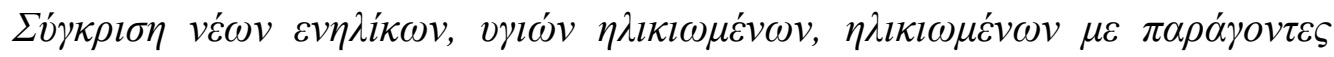

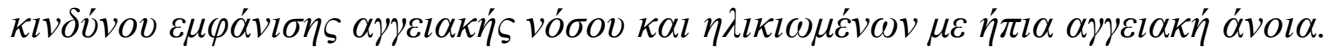

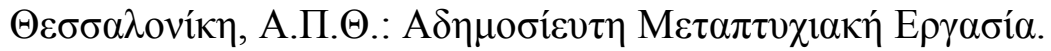

Panza, F., D'Introno, A., Colacicco, A. M., Capurso, C., Pichichero, G., Capurso, S. A., Capurso, A., \& Solfrizzi, V. (2006). Lipid metabolism in cognitive decline and dementia. Brain Research Reviews, 51(2), 275-292.

Paterniti, S., Verdier-Taillefer, M.H., Dufouil C., \& Alperovitch A. (2002). Depressive symptoms and cognitive decline in elderly people. Longitudinal study. The British Journal of Psychiatry, 181, 406-410.

Perner, J. \& Lang, B. (1999). Development of theory of mind and executive control. Trends in Cognitive Neuroscience, 3(9), 337-344.

Perner, J., \& Wimmer, H. (1985). “John thinks that Mary thinks that...”: attribution of second-order false beliefs by 5- to 10-year olds. Journal of Experimental Child Psychology, 39, 437-471.

Peters, R. (2006). Ageing and the brain. Postgraduate Medical Journal, 82(964), 8488. 
Premack, D., \& Woodruff, G. (1978). Does the chimpanzee have a theory of mind? Behavioral and Brain Sciences, 1, 515-526.

Rakoczy, H., Harder-Kasten, A., \& Sturm, L. (2011). The decline of Theory of Mind in old age is (partly) mediated by developmental changes in domain-general abilities. British Journal of Psychology, 103(1), 58-72.

Ramirez, M., Teresi, J. A., Holmes, D., Gurland, B., \& Lantigua, R. (2006). Differential Item Functioning (DIF) and the Mini-Mental State Examination (MMSE): overview, sample, and issues of translation. Medical Care, 44, 95106.

Rankin, K. P., Salazar, A., Gorno-Tempini, M. L., Sollberger, M., Wilson, S. M., Pavlic, D., \& Miller, B. L. (2009). Detecting sarcasm from paralinguistic cues: anatomic and cognitive correlates in neurodegenerative disease.Neuroimage, 47(4), 2005-2015.

Riddle, D. R. (2007). Brain aging: models, methods, and mechanisms. U.S.: CRC Press.

Rubia, K., Russell, T., Overmeyer, S., Brammer, M. J., Bullmore, E. T., Sharma, T. Simmons, Williams, C. R., Giampietro, V., Andrew, C. M., \& Taylor, E. (2001). Mapping motor inhibition: conjunctive brain activations across different versions of go/no-go and stop tasks. Neuroimage, 13(2), 250-261.

Salthouse, T. A. (2004). What and when of cognitive aging. Current Directions in Psychological Science, 13(4), 140-144.

Schultz-Larsen, K., Kreinerc, S., \& Lomholta, R. K. (2007). Mini-Mental Status Examination: Mixed Rasch model item analysis derived two different cognitive dimensions of the MMSE. Journal of Clinical Epidemiology, 60, $268-279$. 
Shamay-Tsoory, S. G., Tomer, R., \& Aharon-Peretz, J. (2005). The neuroanatomical basis of understanding sarcasm and its relationship to social cognition. Neuropsychology, 19(3), 288-300.

Shany-Ur, T., Poorzand, P., Grossman, S. N., Growdon, M. E., Jang, J. Y., Ketelle, R. S., Miller, B. L., \& Rankin, K. P. (2012). Comprehension of insincere communication in neurodegenerative disease: Lies, sarcasm, and Theory of Mind. Cortex, 48(10), 1329-1341.

Sheikh, J. I., \& Yesavage, J. A. (1986). Geriatric Depression Scale (GDS): Recent evidence and development of a shorter version. Clinical Gerontologist, 5, 165173.

Shibata, M., Toyomura, A., Itoh, H., \& Abe, J. I. (2010). Neural substrates of irony comprehension: A functional MRI study. Brain Research, 1308, 114-123.

Shigemori, K., Ohgi, S., Okuyama, E., Shimura, T., \& Schneider, E. (2010). The factorial structure of the Mini Mental State Examination (MMSE) in Japanese dementia patients. BMC Geriatrics, 10(36).

http://www.biomedcentral.com/1471-2318/10/36

Slessor, G., Phillips, L. H., \& Bull, R. (2007). Exploring the specificity of age-related differences in Theory of Mind tasks. Psychology and Aging, 22(3), 639-643.

Spiro, A., \& Brady, C. (2008). Integrating health into cognitive aging research and theory. In S. Hofer and D. Alwin (Eds.), Handbook of cognitive aging: interdisciplinary perspectives (pp. 260-283). Thousand Oaks, California: Sage.

Thomas, A. J., \& O' Brien, J. T. (2008). Depression and cognition in older adults. Current Opinions in Psychiatry, 21(1), 8-13. 
Winner, E. (1988). The point of words: Children's understanding of metaphor and irony. Cambridge, MA: Harvard University Press.

Winner, E., Brownell, H., Happé, F., Blum, A., \& Pincus, D. (1998). Distinguishing lies from jokes: Theory of mind deficits and discourse interpretation in right hemisphere brain-damaged patients. Brain and Language, 62(1), 89-106.

Winner, E., \& Leekam, S. (1991). Distinguishing irony from deception: Understanding the speaker's second order intention. British Journal of Developmental Psychology, 9, 257-270.

Wrench, J. M., Wilson, S. J., Bladin, P. F., \& Reutens, D. C. (2009). Hippocampal volume and depression: insights from epilepsy surgery. Journal of Neurology, Neurosurgery, and Psychiatry, 80, 539-544.

Yesavage, J. A., Brink, T. L., Rose, T. L., Lum, O., Huang, V., Adey, M., \& Leirer, V. O. (1982-1983). Development and validation of a geriatric depression screening scale: a preliminary report. Journal of Psychiatric Research, 17(1), $37-49$. 